\title{
ON THE CONVERGENCE OF PADÉ APPROXIMATIONS FOR GENERALIZED NEVANLINNA FUNCTIONS
}

\author{
M. S. DEREVYAGIN AND V. A. DERKACH
}

\begin{abstract}
We study a stepwise algorithm for solving the indefinite truncated moment problem and obtain the factorization of the matrix describing the solution of this problem into elementary factors. We consider the generalized Jacobi matrix corresponding to Magnus' continuous $P$-fraction that appears in this algorithm and the polynomials of the first and second kind that are solutions of the corresponding difference equation. Weyl functions and the resolution matrices for finite and infinite Jacobi matrices are computed in terms of these polynomials. Convergence of diagonal and paradiagonal Padé approximation for functions from the generalized Nevanlinna class is studied.
\end{abstract}

\section{INTRODUCTION}

The classical moment problem and the problem of rational approximation for Nevanlinna functions are closely related to Chebyshev's theory of continuous fractions [2, 43, 26. On the one hand, the expansion of a Nevanlinna function $\varphi$ in a continuous fraction yields the construction of the Jacobi matrix and the factorization of the solution matrix of a truncated moment problem into elementary factors. On the other hand, partial fractions can be expressed explicitly in terms of the resolutions of the corresponding truncated Jacobi matrices and coincide with diagonal Padé approximations of the function $\varphi$. Therefore, the problem of convergence of diagonal Padé approximations of the function $\varphi$ can be reduced to the problem of the convergence of the resolution for truncated Jacobi matrices. In the present paper we study the problem of rational approximation of generalized Nevanlinna functions introduced in [19] (see also [41, 42]). First we present the idea of the stepwise Chebyshev process applied to the classical moment problem, 2 .

Let $\mathbf{s}=\left\{s_{j}\right\}_{j=0}^{\infty}$ be an infinite sequence of real numbers. The Hamburger moment problem $M(\mathbf{s})$ consists in finding a measure $d \sigma$ on the real line $\mathbb{R}$ such that

$$
\int_{\mathbb{R}} t^{n} d \sigma(t)=s_{n}, \quad n \in \mathbb{Z}_{+}:=\mathbb{N} \cup\{0\} .
$$

A solvability condition of the moment problem $M(\mathbf{s})$ is that the Hankel matrices

$$
S_{n}=\left(s_{j+k}\right)_{j, k=0}^{n}, \quad n \in \mathbb{Z}_{+},
$$

should be nonnegative. The function $\varphi$ corresponding to the measure $d \sigma$,

$$
\varphi(\lambda)=\int_{\mathbb{R}} \frac{d \sigma(t)}{t-\lambda},
$$

belongs to the Nevanlinna class $\mathbf{N}$ of functions that are holomorphic in the upper halfplane $\mathbb{C}_{+}$and have a nonnegative imaginary part there. It is known ([36]) that each

2000 Mathematics Subject Classification. Primary 30E05; Secondary 47A57. 
function of the class $\mathbf{N}$ satisfying the additional condition

$$
\varphi(\lambda)=O\left(\frac{1}{\lambda}\right) \quad(\lambda \widehat{\rightarrow} \infty)
$$

admits the integral representation (1.3). In the sequel, the notation $\lambda \widehat{\rightarrow} \infty$ means that $\lambda$ tends to $\infty$ nontangentially remaining inside a sector $\delta<\arg \lambda<\pi-\delta$ for some $\delta>0$. According to the Hamburger-Nevanlinna theorem (see [2]), the classical moment problem $M(\mathbf{s})$ is equivalent to the asymptotic expansions

$$
\varphi(\lambda)=-\frac{s_{0}}{\lambda}-\frac{s_{1}}{\lambda^{2}}-\cdots-\frac{s_{2 n}}{\lambda^{2 n+1}}+o\left(\frac{1}{\lambda^{2 n+1}}\right) \quad(\lambda \widehat{\rightarrow} \infty)
$$

for each $n \in \mathbb{N}$. Therefore, the moment problem (1.1) can be viewed as a boundary interpolation problem with an infinite number of interpolation points. The truncated moment problem consists in finding functions $\varphi \in \mathbf{N}$ such that the expansion (1.5) holds for a given $M(\mathbf{s}, n)$. The set of solutions of the truncated moment problem $M(\mathbf{s}, n)$ can be described using the Chebyshev algorithm for the expansion of a function $\varphi \in \mathbf{N}$ in a continuous fraction. Let $\varphi_{0}:=\varphi \in \mathbf{N}$ admit an expansion (1.5) with $s_{0}=1$. Then the function $-\frac{1}{\varphi_{0}}$ also belongs to $\mathbf{N}$ and admits the expansion

$$
-\frac{1}{\varphi_{0}(\lambda)}=\lambda-a_{0}+b_{0}^{2} \varphi_{1}(\lambda)
$$

where $a_{0}=\bar{a}_{0}, b_{0}>0$, and $\varphi_{1}$ is a function of class $\mathbf{N}$ with the asymptotic expansion

$$
\varphi_{1}(\lambda)=-\frac{s_{0}^{(1)}}{\lambda}-\frac{s_{1}^{(1)}}{\lambda^{2}}-\cdots-\frac{s_{2(n-1)}^{(1)}}{\lambda^{2 n-1}}+o\left(\frac{1}{\lambda^{2 n-1}}\right) \quad(\lambda \widehat{\rightarrow} \infty)
$$

with $s_{0}^{(1)}=1$ and some $s_{i}^{(1)} \in \mathbb{R}, i=1, \ldots, 2 n-2$.

Continuing this process we construct a sequence of functions $\varphi_{j} \in \mathbf{N}, j=0,1, \ldots, n$, connected by the fractional linear transformations

$$
\varphi_{j}(\lambda)=T_{j}\left[\varphi_{j+1}(\lambda)\right]:=\frac{-1}{\lambda-a_{j}+b_{j}^{2} \varphi_{j+1}(\lambda)} \quad(j=0,1, \ldots, n-1),
$$

$a_{j}=\bar{a}_{j}, b_{j}>0, j=0,1, \ldots, n$. Therefore, with the infinite moment problem one can associate a continuous fraction

$$
-\frac{1}{\lambda-a_{0}}-\frac{b_{0}^{2}}{\lambda-a_{1}}-\frac{b_{1}^{2}}{\lambda-a_{2}}-\cdots
$$

Let us consider the infinite and the truncated Jacobi matrices $H$ and $H_{[0, n-1]}$ :

$$
H=\left(\begin{array}{cccc}
a_{0} & b_{0} & & \\
b_{0} & a_{1} & b_{1} & \\
& b_{1} & a_{2} & \ddots \\
& & \ddots & \ddots
\end{array}\right), \quad H_{[0, n-1]}=\left(\begin{array}{cccc}
a_{0} & b_{0} & & \\
b_{0} & a_{1} & \ddots & \\
& \ddots & \ddots & b_{n-2} \\
& & b_{n-2} & a_{n-1}
\end{array}\right) .
$$

The set of solutions of the truncated moment problem $M(\mathbf{s}, n-1)$ is described by the formula

$$
\varphi(\lambda)=T_{0} \circ T_{1} \circ \cdots \circ T_{n-1}\left[\varphi_{n}(\lambda)\right],
$$

where $\varphi_{n}(\lambda)$ is a function of class $\mathbf{N}$ satisfying the Nevanlinna condition

$$
\lim _{\lambda \leadsto \infty} \frac{\varphi_{n}(\lambda)}{\lambda}=0 .
$$


The matrix $\mathcal{W}_{[0, n-1]}$ of coefficients of the fractional linear transformation (1.10) can be computed explicitly in terms of the polynomials of the first and second kind, $P_{j}(\lambda)$ and $Q_{j}(\lambda)$, corresponding to the Jacobi matrix $H$ :

$$
\mathcal{W}_{[0, n-1]}(\lambda)=\left(\begin{array}{rr}
-b_{n-1} Q_{n-1}(\lambda) & -Q_{n}(\lambda) \\
b_{n-1} P_{n-1}(\lambda) & P_{n}(\lambda)
\end{array}\right)
$$

Therefore, solutions of the truncated moment problem $M(\mathbf{s}, n-1)$ take the form

$$
\varphi(\lambda)=-\frac{b_{n-1} Q_{n-1}(\lambda) \tau(\lambda)+Q_{n}(\lambda)}{b_{n-1} P_{n-1}(\lambda) \tau(\lambda)+P_{n}(\lambda)} .
$$

One of the solutions of the truncated moment problem $M(\mathbf{s}, n-1)$ is the $n$-th partial fraction $-\frac{Q_{n}(\lambda)}{P_{n}(\lambda)}$ for (1.9), which admits the expansion (see [2, 26])

$$
-\frac{Q_{n}(\lambda)}{P_{n}(\lambda)}=-\frac{s_{0}}{\lambda}-\frac{s_{1}}{\lambda^{2}}-\cdots-\frac{s_{2 n-1}}{\lambda^{2 n}}+O\left(\frac{1}{\lambda^{2 n+1}}\right) \quad(\lambda \widehat{\rightarrow} \infty)
$$

and can be represented in the form

$$
-\frac{Q_{n}(\lambda)}{P_{n}(\lambda)}=\left(\left(H_{[0, n-1]}-\lambda\right)^{-1} e, e\right),
$$

where $e=(1,0, \ldots, 0)^{\top} \in \mathbb{C}^{n}$. In the case where the moment problem is definite, i.e., the Jacobi matrix $H$ defines a selfadjoint operator in $\ell_{2}$, the moment problem $M(\mathbf{s})$ has a unique solution, which is of the form

$$
\varphi(\lambda)=\left((H-\lambda)^{-1} e, e\right)_{\ell_{2}} .
$$

Formulas (1.5) and (1.12) imply a remarkable fact that

$$
\varphi(\lambda)+\frac{Q_{n}(\lambda)}{P_{n}(\lambda)}=O\left(\frac{1}{\lambda^{2 n+1}}\right) \quad(\lambda \widehat{\rightarrow} \infty),
$$

i.e., the rational function $-\frac{Q_{n}(\lambda)}{P_{n}(\lambda)}$ is the $n$-th diagonal Padé approximation for the function $\varphi(\lambda)$ (see Definition 4.5). For functions of the form

$$
\varphi(\lambda)=\widehat{\sigma}(\lambda):=\int_{a}^{b} \frac{d \sigma(t)}{t-\lambda},
$$

strong convergence of resolutions $\left(H_{[0, n-1]}-\lambda\right)^{-1}$ to $(H-\lambda)^{-1}$ implies, according to (1.13) and (1.14), the uniform convergence in $\mathbb{C} \backslash[a, b]$ of the diagonal Padé approximations $-\frac{Q_{n}(\lambda)}{P_{n}(\lambda)}$ to the function $\varphi(\lambda)$. This is the classical theorem of A. A. Markov, 9, 43].

In the papers by A. A. Gonchar [14 and E. A. Rahmanov [44, Markov's result was generalized to "rational perturbations"

$$
\varphi(\lambda)=\widehat{\sigma}(\lambda)+r(\lambda)
$$

of a Markov function of type (1.15). In particular, in 44 it is shown that in the case where $r$ is a regular rational real fraction that is holomorphic on the interval $[a, b]$, the $n$-th diagonal Padé approximations exist for all sufficiently large $n$ and converge to $\varphi$ locally uniformly on $\mathbb{C} \backslash([a, b] \cup \mathcal{P}(\varphi))$, where $\mathcal{P}(\varphi)$ is the set of poles of $\varphi$.

For a long time many authors were interested in the Baker-Gammel-Wills conjecture (also known as the Padé conjecture), which as formulated in [8]. This conjecture asks whether a function that is meromorphic in the unit circle has a locally uniformly converging subsequence of the diagonal Padé approximation. However, in the paper [40] by D. Lubinsky a nice counterexample to this conjecture was constructed using the RogersRamanujan function. A significantly simpler counterexample to the Padé conjecture was presented by V. I. Buslaev in [11] using a hyperelliptic function that can be expanded in a periodic continuous fraction. 
In this paper we study the convergence of diagonal Padé approximations for "rational perturbations" of Markov functions of the form

$$
\varphi(\lambda)=r_{1}(\lambda) \widehat{\sigma}(\lambda)+r_{2}(\lambda) .
$$

The main result of the paper is the following theorem.

Theorem 1.1. Let $\widehat{\sigma}$ be a Markov function of the form (1.15), $r_{1}=q / \omega, \operatorname{deg} q \leq \operatorname{deg} \omega$, a real rational function that is nonnegative on $\mathbb{R} \backslash \mathcal{P}\left(r_{1}\right)$, and $r_{2}$ a regular rational real function. Then the $n$-th diagonal Padé approximations for the function of the form (1.17) exist for all sufficiently large $n$ and converge locally uniformly to $\varphi(\lambda)$ in $\mathbb{C} \backslash([a, b] \cup \mathcal{P}(\varphi))$.

Meromorphic functions of the form (1.17) belong to the class of generalized Nevanlinna functions introduced in 30. Every generalized Nevanlinna function can be represented as a $Q$-function (see [31, 39]) or as a Weyl function (see [21]) of a symmetric operator acting in the Pontryagin space. In particular, it is shown in [20 that every function of the form (1.17) can be represented as the $m$-function of a generalized Jacobi matrix that is a block-tridiagonal matrix of a special form. Such a matrix is naturally related to the $P$-fraction of the form

$$
-\frac{\varepsilon_{0}}{p_{0}(\lambda)}-\frac{\varepsilon_{0} \varepsilon_{1} b_{0}^{2}}{p_{1}(\lambda)}-\cdots-\frac{\varepsilon_{N-1} \varepsilon_{N} b_{N-1}^{2}}{p_{N}(\lambda)}-\cdots
$$

that appears in the stepwise Chebyshev process applied to a generalized Nevanlinna function $\varphi$ of the class $\mathbf{N}_{\kappa}$. A general definition of a $P$-fraction was introduced by Magnus in [4] (see also [26]).

It is well known (see [41, 26]) that the partial fraction $-Q_{n}(\lambda) / P_{n}(\lambda)$ in (1.18) is the $n$-th diagonal Padé approximation of the function $\varphi$. Under the hypotheses of Theorem 1.1 the convergence of this approximation can be deduced from the fact that the truncated generalized Jacobi matrices $H_{[0, n]}$ converge to $H$ in the strong resolution sense. This approach works also in the case where the measure $d \sigma$ in (1.17) does not have compact support but still corresponds to a definite moment problem. In the case where $d \sigma$ corresponds to an indefinite moment problem, it is shown that Theorem 1.1 remains true for a subsequence of diagonal approximations of the function $\varphi$. Let us note that this approach to the proof of the convergence of diagonal Padé approximations was introduced in [45] and for tridiagonal complex matrices in [7, 1] (see also 47]). However, an explicit form of $m$-functions corresponding to the generalized Jacobi matrix was not presented in either [7] or [1].

In addition to diagonal approximations we study the convergence of paradiagonal Padé approximations for functions of class (1.17). In solving these problems an important role is played by the factorization of the matrix $\mathcal{W}_{[0, n]}$ of solutions of the truncated moment problem. This factorization is obtained by elementary methods in 2.3 . However, taking into account the operator approach used in the paper, we decided to present in Appendix A another proof of the factorization theorem based on the multiplication theorem for characteristic functions and on the fact that $\mathcal{W}_{[0, n]}(\lambda)$ is the characteristic function of a linear relation associated to the matrix $H_{[0, n]}$.

The paper is organized as follows. In Section 2 we introduce the class $\mathbf{N}_{\kappa}$ of generalized Nevanlinna functions and describe the Chebyshev algorithm as applied to this class. We obtain a description of solutions of the truncated moment problem in the class $\mathbf{N}_{\kappa}$ and the factorization of the solution matrix $\mathcal{W}_{[0, n]}$. In Section 3 we introduce the generalized Jacobi matrices and compute the Weyl functions and resolution matrices of symmetric operators associated to the matrix $H$. We also characterize the class of resolution matrices associated with generalized Jacobi matrices. In Section 4 we present a result on the convergence of resolution matrices for the truncated Jacobi matrices and 
prove the main theorem of the paper concerning the convergence of both diagonal and paradiagonal Padé approximations. Finally, in Appendix A we prove that the resolution matrices $\mathcal{W}_{[0, n]}$ are the characteristic functions of certain linear relations in the sense of 22]. As a corollary of the results in 22] we obtain a theorem on the factorization of the resolution matrix $\mathcal{W}_{[0, n]}$.

\section{Functions in the generalized Nevanlinna Class}

2.1. Integral representation. Let $\Omega$ be a subset of the complex plane $\mathbb{C}$ and let the kernel $\mathrm{N}(\lambda, \mu)$ be defined on $\Omega \times \Omega$. We say that the kernel $\mathrm{N}(\lambda, \mu)$ has $\kappa$ negative squares in $\Omega\left(\kappa \in \mathbb{Z}_{+}\right)$if it is Hermitian, i.e., $\overline{\mathrm{N}(\lambda, \mu)}=\mathrm{N}(\mu, \lambda)$ for all $\lambda, \mu \in \Omega$ and for each collection of numbers $n \in \mathbb{N}, \lambda_{1}, \lambda_{2}, \ldots, \lambda_{n} \in \Omega$, the Hermitian matrix $\left(\mathrm{N}\left(\lambda_{i}, \lambda_{j}\right)\right)_{i, j=1}^{n}$ has at most $\kappa$ negative eigenvalues, and for some $\lambda_{1}, \lambda_{2}, \ldots, \lambda_{n} \in \Omega$ it has exactly $\kappa$ eigenvalues (counted with multiplicities).

Let us recall ([34] $)$ that a function $\varphi$ meromorphic in $\mathbb{C}_{+}$belongs to the generalized Nevanlinna class $\mathbf{N}_{\kappa}$ if the kernel

$$
\mathrm{N}_{\varphi}(\lambda, \mu)=\frac{\varphi(\lambda)-\overline{\varphi(\mu)}}{\lambda-\bar{\mu}}
$$

has $\kappa$ negative squares on $\mathbb{C}_{+} \backslash \mathcal{P}(\varphi)$ where $\mathcal{P}(\varphi)$ is the set of poles of the function $\varphi$.

It is known ( $[35)$ that each function $\varphi$ of the class $\mathbf{N}_{\kappa}$ has at most $\kappa$ poles in $\mathbb{C}_{+}$. To make this statement more precise, recall ([38) that a point $\alpha \in \mathbb{R}$ is called a generalized multiplicity $\kappa_{\alpha}$ pole of negative type of a function $\varphi$ if

$$
-\infty<\lim _{\lambda \rightarrow \alpha}(\lambda-\alpha)^{2 \kappa_{\alpha}+1} \varphi(\lambda) \leq 0, \quad 0<\lim _{\lambda \rightrightarrows \alpha}(\lambda-\alpha)^{2 \kappa_{\alpha}-1} \varphi(\lambda) \leq \infty .
$$

Similarly, the point $\infty$ is called a generalized multiplicity $\kappa_{\infty}$ pole of negative type of a function $\varphi$ if

$$
0 \leq \lim _{\lambda \leadsto \infty} \frac{\varphi(\lambda)}{\lambda^{2 \kappa_{\infty}+1}}<\infty, \quad-\infty \leq \lim _{\lambda \rightrightarrows \infty} \frac{\varphi(\lambda)}{\lambda^{2 \kappa_{\infty}-1}}<0 .
$$

In [35] it is shown that for a function $\varphi$ of class $\mathbf{N}_{\kappa}$ the total number of poles in $\mathbb{C}_{+}$ and generalized poles in $\mathbb{R} \cup\{\infty\}$ equals $\kappa$. For example, a real polynomial $p(\lambda)=$ $p_{k} \lambda^{k}+\cdots+p_{1} \lambda+p_{0}$ has a unique generalized pole in $\infty$ and according to (2.2), $p \in \mathbf{N}_{\kappa_{-}(p)}$, where

$$
\kappa_{-}(p):= \begin{cases}{[k / 2]+1} & \text { if } k \text { is odd and } p_{k}<0 \\ {[k / 2]} & \text { otherwise. }\end{cases}
$$

Later we will be interested in functions of class $\mathbf{N}_{\kappa}$ satisfying (1.4). For such functions the point $\infty$ is not a generalized pole of negative type. We say (see [24]) that a function $\varphi$ of class $\mathbf{N}_{\kappa}$ belongs to the class $\mathbf{N}_{\kappa,-2 n}$ if the expansion (1.5) holds for some $s_{0}, s_{1}, \ldots, s_{2 n} \in$ $\mathbb{R}$. Next let

$$
\mathbf{N}_{\kappa,-\infty}:=\bigcap_{n \geq 0} \mathbf{N}_{\kappa,-2 n}
$$

The next proposition follows from [32, Satz 3.1] and [24, Theorem 3.3] (see also [18]).

Proposition 2.1. Each function $\varphi$ of class $\mathbf{N}_{\kappa,-\infty}$ admits an integral representation

$$
\varphi(\lambda)=r_{1}(\lambda) \int_{\mathbb{R}} \frac{d \sigma(t)}{t-\lambda}+r_{2}(\lambda),
$$

where $d \sigma$ is a finite measure $\mathbb{R}$ with finite moments of all orders, $r_{1}(\lambda)=q(\lambda) / \omega(\lambda)$ is a rational function nonnegative on $\mathbb{R} \backslash \mathcal{P}\left(r_{1}\right)$ such that $\operatorname{deg} q \leq \operatorname{deg} \omega$, and $r_{2}(\lambda)$ is a regular rational real function. Among representations of the form (2.4) there is exactly one, the so-called irreducible representation, characterized by the property that all zeros of 
the function $\omega$ coincide with generalized poles of negative type of the function $\varphi$, counted with multiplicities, and $q(\lambda) \equiv 1$.

The converse result is also true: each function $\varphi$ of type (2.4) belongs to the class $\mathbf{N}_{\kappa,-\infty}$ for some $\kappa \in \mathbb{Z}_{+}$.

Everywhere below we will extend a function $\varphi$ of the class $\mathbf{N}_{\kappa,-\infty}$ to $\mathbb{C}_{-}$by the formula

$$
\varphi(\lambda):=\overline{\varphi(\bar{\lambda})}, \quad \lambda \in \mathbb{C}_{-} .
$$

This agrees with the integral representation (2.4).

2.2. Operator representation of functions of the class $\mathbf{N}_{\kappa}$. Functions of class $\mathbf{N}_{\kappa}$ naturally occur in the spectral theory of selfadjoint operators in Pontryagin spaces.

Let us recall $([28])$ that a pair $(\mathfrak{H},[\cdot, \cdot])$ consisting of a linear space $\mathfrak{H}$ and a sesquilinear form $[\cdot, \cdot]$ on $\mathfrak{H} \times \mathfrak{H}$ is called a space with indefinite metric. A linear subspace $\mathfrak{L}$ in $\mathfrak{H}$ is called positive (resp., negative) if $[f, f]>0$ (resp., $[f, f]<0$ ) for all $f \in \mathfrak{H}, f \neq 0$. A space with indefinite metric $(\mathfrak{H},[\cdot, \cdot])$ is called a Pontryagin space if it can be decomposed in the orthogonal (with respect to the form $[\cdot, \cdot]$ ) sum

$$
\mathfrak{H}=\mathfrak{H}_{+}[+] \mathfrak{H}_{-}
$$

of a positive space $\left(\mathfrak{H}_{+},[\cdot, \cdot]\right)$ and a negative space $\mathfrak{H}_{-}$of finite dimension $\kappa$ called the negative index of the space $\mathfrak{H}$ and denote ind $\mathfrak{H}_{-}=\kappa$.

For a linear operator $A$ in $(\mathfrak{H},[\cdot, \cdot])$ with dense domain $\operatorname{dom} A$ define a selfadjoint operator $A^{+}$by the formula

$$
A^{+} g=g^{\prime} \quad \text { if }[A f, g]=\left[f, g^{\prime}\right] \text { for all } f \in \operatorname{dom} A .
$$

A closed linear operator $A$ in $(\mathfrak{H},[\cdot, \cdot])$ is called selfadjoint (symmetric) if $A=A^{+}(A \subset$ $A^{+}$). The set of regular points, the spectrum, and the point spectrum of the operator $A$ are denoted by $\rho(A), \sigma(A)$, and $\sigma_{p}(A)$ respectively.

One can easily see that if $\widetilde{A}$ is a selfadjoint operator in a Pontryagin space with negative index $\kappa$ and $u \in \mathfrak{H}$ is a generating vector for $A$, i.e.,

$$
\overline{\operatorname{span}}\left\{(\widetilde{A}-\lambda)^{-1} u: \lambda \in \rho(\widetilde{A})\right\}=\mathfrak{H},
$$

then the function

$$
\varphi(\lambda)=\left[(\widetilde{A}-\lambda)^{-1} u, u\right]
$$

belongs to the class $\mathbf{N}_{\kappa}$. If, in addition, $u \in \bigcap_{n>0} \operatorname{dom} \widetilde{A}^{n}$, then the function $\varphi$ belongs to the class $\mathbf{N}_{\kappa,-\infty}$. The converse statement also holds.

Theorem $2.2(32,24)$. Each function of the class $\mathbf{N}_{\kappa,-\infty}$ admits an operator representation (2.6) with a selfadjoint operator $\widetilde{A}$ in a Pontryagin space and a generating vector $u \in \bigcap_{n \geq 0} \operatorname{dom} \widetilde{A}^{n}$. Among various representations of the form (2.6) of the function $\varphi$ there is exactly one (up to unitary equivalence) representation for which $u$ is a cyclic vector and ind $-\mathfrak{H}=\kappa$.

Representation (2.6) implies the following relations between the "moments" $s_{n}$ in the asymptotic expansion (1.5) of the function $f$ and the powers of the operator $\widetilde{A}$ :

$$
s_{n}=\left[\widetilde{A}^{n} u, u\right], \quad n \in \mathbb{Z}_{+} .
$$

The operator $\widetilde{A}$ in the representation (2.6) can be viewed as a selfadjoint extension of the symmetric operator $A$ defined as the restriction of the operator $\widetilde{A}$ to the set

$$
\operatorname{dom} A=\{x \in \mathfrak{H}:[x, u]=0\} .
$$


Let us recall that a point $\lambda \in \mathbb{C}$ is called a point of regular type of a symmetric operator $A$ in a Pontryagin space $\mathfrak{H}$, denoted $\lambda \in \widehat{\rho}(A)$, if $\lambda \notin \sigma_{p}(A)$ and $\operatorname{ran}(A-\lambda)$ is closed in $\mathfrak{H}$. It is known (see [30]) that the set $\widehat{\rho}(A)$ of points of regular type contains all but a finite number of points in $\mathbb{C}_{ \pm}$and the dimension of the space $\mathfrak{N}_{\lambda}:=\mathfrak{H}[-] \operatorname{ran}(A-\bar{\lambda})$ takes a constant value $n_{ \pm}(A)$ for all $\lambda \in \mathbb{C}_{ \pm} \cap \widehat{\rho}(A)$. The subspaces $\mathfrak{N}_{\lambda}:=\mathfrak{H}[-] \operatorname{ran}(A-\bar{\lambda})$ are called the defect subspaces and the numbers $n_{ \pm}(A):=\operatorname{dim} \mathfrak{N}_{\lambda}, \lambda \in \mathbb{C}_{ \pm} \cap \widehat{\rho}(A)$, the defect numbers of a symmetric operator $A$. A symmetric operator $A$ in a Pontryagin space $\mathfrak{H}$ is called simple if

$$
\overline{\operatorname{span}}\left\{\mathfrak{N}_{\lambda}: \lambda \in \mathbb{C}_{ \pm} \cap \widehat{\rho}(A)\right\}=\mathfrak{H} .
$$

It is clear that the operator $A$ defined by (2.8) has the defect numbers $(1,1)$ and its defect subspaces are given by the formula

$$
\mathfrak{N}_{\lambda}=\operatorname{span}\left\{(\widetilde{A}-\lambda)^{-1} u\right\}, \quad \lambda \in \widehat{\rho}(A) .
$$

Formula (2.9) implies that the operator $A$ is simple in the case where $u$ is a generating vector for $\widetilde{A}$. Let us emphasize that in this case the function $\varphi$ defined by (2.6) completely characterizes the spectrum of the operator $\widetilde{A}$ and is the Weyl function of the operator $A$ in the sense of definition (3.29). Operator representations of the form (2.6) for a certain class of operators associated with the indefinite moment problem are studied in Section 3.

2.3. Indefinite moment problem. In 1979, M. G. Krein and H. Langer 34 considered the following indefinite analog of the Hamburger moment problem.

Problem $M_{\kappa}(\mathbf{s})$. Suppose we are given a sequence $\mathbf{s}=\left\{s_{j}\right\}_{j=0}^{\infty}, \kappa \in \mathbb{Z}_{+}$. Find $\varphi \in$ $\mathbf{N}_{\kappa,-\infty}$ with the asymptotic expansion (1.5) for each $n \in \mathbb{N}$.

At was shown in [34, for the solvability of Problem $M_{\kappa}(\mathbf{s})$ it is necessary and sufficient that for each $n \in \mathbb{N}$, the Hankel matrix of the form (1.2) has at most $\kappa$ negative eigenvalues. The necessity of this condition follows, for example, from the representation (2.7) for moments $s_{j}$. Indeed, if a function $\varphi \in \mathbf{N}_{\kappa,-\infty}$ is a solution of the problem $M_{\kappa}(\mathbf{s})$, then it admits the operator representation (2.6) and the moments $s_{k}$ in the asymptotic expansion (1.7) are of the form (2.7). Since ind $-\mathfrak{H}=\kappa$, the form

$$
\sum_{j, k=0}^{n} s_{j+k} \xi_{j} \bar{\xi}_{k}=\sum_{j, k=0}^{n}\left[\widetilde{A}^{j+k} u, u\right] \xi_{j} \bar{\xi}_{k}=\left[\sum_{j=0}^{n} \xi_{j} \widetilde{A}^{j} u, \sum_{j=0}^{n} \xi_{j} \widetilde{A}^{j} u\right]
$$

has at most $\kappa$ negative eigenvalues.

Together with Problem $M_{\kappa}(\mathbf{s})$ we will consider the truncated problem $M_{\kappa}(\mathbf{s}, n)$, where one has to find $\varphi \in \mathbf{N}_{\kappa,-2 n}$ satisfying (1.5) for a given $n \in \mathbb{N}$. Everywhere in this paper we will consider the nondegenerate moment problem characterized by the condition $\operatorname{det} S_{n} \neq 0$.

One of the methods used to solve this problem is based on the stepwise Chebyshev method explained earlier. In [19 this method, called there the Schur algorithm, is adapted to the indefinite moment problem. Let us present the stepwise algorithm for the problem $M_{\kappa}(\mathbf{s}, n)$. It relies on the following properties of functions belonging to generalized Nevanlinna classes (see [32]):

(i) if $\varphi \in \mathbf{N}_{\kappa}$, then $-\frac{1}{\varphi} \in \mathbf{N}_{\kappa}$;

(ii) if $\varphi \in \mathbf{N}_{\kappa_{1}}$ satisfies the condition (1.4) and $p \in \mathbf{N}_{\kappa_{2}}$ is a real polynomial, then $\varphi+p \in \mathbf{N}_{\kappa_{1}+\kappa_{2}}$.

Further on in this paper we assume that the sequence $\mathbf{s}$ is normalized, i.e., the first nonzero moment has absolute value 1 . Let $n_{1}<n_{2}<\cdots<n_{N+1}=n+1$ be the sequence 
of all normal indices of the matrix $S_{n}$, i.e., the numbers $j \in \mathbb{N}$ such that $\operatorname{det} S_{j-1} \neq 0$, and let the numbers $\kappa_{0} \leq \kappa_{1} \leq \cdots \leq \kappa_{N}$ be defined by the formulas

$$
\kappa_{j}=\mathrm{sq}_{-} S_{n_{j+1}-1}, \quad j=0,1, \ldots, N .
$$

By the choice of $n_{1}$ we have $s_{n_{1}-1} \neq 0$. Let $\varepsilon_{0}=s_{n_{1}-1},\left|\varepsilon_{0}\right|=1$, and let the function $\varphi_{0}:=\varphi \in \mathbf{N}_{\kappa,-2 n}$ satisfy (1.5). By property (i) the function $-\frac{1}{\varphi_{0}}$ also belongs to $\mathbf{N}_{\kappa}$ and the principal part of the asymptotic expansion of the function $-\frac{1}{\varphi_{0}}$ at the point $\infty$ is a polynomial of degree $k_{0}:=n_{1}$ with the leading coefficient $\varepsilon_{0}$. Therefore there exists a polynomial $p_{0}$ of degree $k_{0}$ such that the function $\varphi_{1}$ defined by the formula

$$
-\frac{1}{\varphi_{0}(\lambda)}=\varepsilon_{0} p_{0}(\lambda)+b_{0}^{2} \varphi_{1}(\lambda), \quad b_{0}>0
$$

satisfies condition (1.4). Choose $b_{0}>0$ such that the sequence

$$
\mathbf{s}^{(1)}=\left\{s_{j}^{(1)}\right\}_{j=0}^{\infty},
$$

defined from the expansion

$$
\varphi_{1}(\lambda)=-\frac{s_{0}^{(1)}}{\lambda}-\frac{s_{1}^{(1)}}{\lambda^{2}}-\cdots-\frac{s_{2\left(n-k_{0}\right)}^{(1)}}{\lambda^{2\left(n-k_{0}\right)+1}}+o\left(\frac{1}{\lambda^{2\left(n-k_{0}\right)+1}}\right) \quad(\lambda \widehat{\rightarrow} \infty),
$$

is normalized. Property (ii) implies that $\varphi_{1} \in \mathbf{N}_{\kappa^{\prime}}$ with $\kappa^{\prime}=\kappa-\kappa_{-}\left(\varepsilon_{0} p_{0}\right)$. Let us note that the index $\kappa_{-}\left(\varepsilon_{0} p_{0}\right)$ defined by (2.3) coincides with the number $\kappa_{0}=\mathrm{sq}_{-} S_{k_{0}-1}$ of negative squares of the triangular matrix

$$
S_{n_{1}-1}=S_{k_{0}-1}=\varepsilon_{0}\left(\begin{array}{lll}
\mathbf{0} & & 1 \\
& . & \\
1 & & *
\end{array}\right) .
$$

Therefore, $\varphi_{1} \in \mathbf{N}_{\kappa-\kappa_{0}}$. This completes the construction of the first step of the induction. form

According to the results in [19], the set of normal indices of the matrix $S_{n-k_{0}}^{(1)}$ is of the

$$
n_{2}-k_{0}<\cdots<n_{N+1}-k_{0},
$$

and sq_ $S_{n_{j}-k_{0}-1}^{(1)}=\kappa_{j}-\kappa_{0}$. Inductively, we construct a sequence of functions $\varphi_{j} \in$ $\mathbf{N}_{\kappa-\kappa_{j-1}}$ (for convenience, here and later we assume that $\kappa_{-1}=0$ ) satisfying the conditions

$$
\varphi_{j}(\lambda)=T_{j}\left[\varphi_{j+1}(\lambda)\right]:=-\frac{\varepsilon_{j}}{p_{j}(\lambda)+\varepsilon_{j} b_{j}^{2} \varphi_{j+1}(\lambda)}, \quad j=0,1, \ldots, N,
$$

where the $p_{j}$ are normalized real polynomials of degree $k_{j}, \varepsilon_{j}= \pm 1, b_{j}>0$. Conditions (2.13) and $\varphi_{j} \in \mathbf{N}_{\kappa-\kappa_{j-1}}$ imply that

$$
\varphi_{j+1} \in \mathbf{N}_{\kappa^{\prime}}, \quad \kappa^{\prime}=\kappa-\kappa_{j-1}-\kappa_{-}\left(\varepsilon_{j} p_{j}\right)=\kappa-\kappa_{j-1}-\mathrm{sq}_{-} S_{k_{j}-1}^{(j)},
$$

where the elements of the Hankel matrix $S_{m}^{(j)}=\left(s_{i+l}^{(j)}\right)_{i, l=0}^{m}$ are the coefficients of the expansion (1.5) for the function $\varphi_{j}$. Taking into account the relation sq_ $S_{k_{j}-1}^{(j)}=\kappa_{j}-$ $\kappa_{j-1}$ (see [19]) we see that $\varphi_{j+1} \in \mathbf{N}_{\kappa-\kappa_{j}}$.

After $N+1$ steps we obtain the following representation for the function $\varphi_{0}(\lambda)$ :

$$
\begin{aligned}
\varphi_{0}(\lambda) & =T_{0} \circ T_{1} \circ \cdots \circ T_{N}\left[\varphi_{N+1}(\lambda)\right] \\
& =-\frac{\varepsilon_{0}}{p_{0}(\lambda)}-\frac{\varepsilon_{0} \varepsilon_{1} b_{0}^{2}}{p_{1}(\lambda)}-\ldots-\frac{\varepsilon_{N-1} \varepsilon_{N} b_{N-1}^{2}}{p_{N}(\lambda)+\varepsilon_{N} b_{N}^{2} \varphi_{N+1}(\lambda)},
\end{aligned}
$$

where the polynomial $p_{N}$ is determined uniquely up to an additive constant, and the function $\varphi_{N+1} \in \mathbf{N}_{\kappa-\kappa_{N}}$ satisfies the Nevanlinna condition (1.4). 
The matrices of the fractional linear transformations $T_{j}$ in (2.15) have the form

$$
\mathcal{W}_{j}(\lambda)=\left(\begin{array}{cc}
0 & -\frac{\varepsilon_{j}}{b_{j}} \\
\varepsilon_{j} b_{j} & \frac{p_{j}(\lambda)}{b_{j}}
\end{array}\right), \quad j \in \mathbb{Z}_{+} .
$$

Therefore their composition $T_{0} \circ T_{1} \circ \cdots \circ T_{N}$ corresponds to the product of the matrices $\mathcal{W}_{j}(\lambda)$ :

$$
\mathcal{W}_{[0, N]}(\lambda)=\left(w_{i j}(\lambda)\right)_{i, j=1}^{2}:=\mathcal{W}_{0}(\lambda) \mathcal{W}_{1}(\lambda) \ldots \mathcal{W}_{N}(\lambda)
$$

Summarizing, we obtain the following description of the solution of the truncated moment problem $M_{\kappa}(\mathbf{s}, n)$, which appeared before in several different forms in [27, 21, 19].

Theorem 2.3. Let $\kappa \in \mathbb{Z}_{+}$and let $\mathbf{s}=\left\{s_{j}\right\}_{j=0}^{2 n}$ be a sequence of integers such that

$$
\operatorname{det} S_{n} \neq 0, \quad \text { sq_ } S_{n} \leq \kappa .
$$

Let $n_{1}<n_{2}<\cdots<n_{N+1}=n+1$ be a sequence of all normal indices of the matrix $S_{n}$ and let the numbers $\kappa_{j}(j=0,1, \ldots, N)$ and the matrix $\mathcal{W}_{[0, N]}$ be defined by formulas (2.10) and (2.17). Then the set of all solutions $\varphi \in \mathbf{N}_{\kappa}$ of the truncated indefinite problem $M_{\kappa}(\mathbf{s}, n)$ is described by the formula

$$
\varphi(\lambda)=\frac{w_{11}(\lambda) \tau(\lambda)+w_{12}(\lambda)}{w_{21}(\lambda) \tau(\lambda)+w_{22}(\lambda)}
$$

where $\tau$ runs over the set of functions in $\mathbf{N}_{\kappa-\kappa_{N}}$ satisfying the Nevanlinna condition (1.11).

Let us present an explicit formula for the matrix of solutions $\mathcal{W}_{[0, N]}$ of the problem $M_{\kappa}(\mathbf{s}, n)$ in terms of the parameters $p_{j}, b_{j}, \varepsilon_{j}$ that occur in the Chebyshev algorithm. First define the polynomials $P_{j+1}(\lambda), Q_{j+1}(\lambda)$ by the formulas

$$
\left(\begin{array}{r}
-Q_{0} \\
P_{0}
\end{array}\right)=\left(\begin{array}{l}
0 \\
1
\end{array}\right), \quad\left(\begin{array}{r}
-Q_{j+1}(\lambda) \\
P_{j+1}(\lambda)
\end{array}\right):=\mathcal{W}_{[0, j]}(\lambda)\left(\begin{array}{l}
0 \\
1
\end{array}\right), \quad j \in \mathbb{Z}_{+} .
$$

Then the formula $\mathcal{W}_{[0, j]}(\lambda)=\mathcal{W}_{[0, j-1]}(\lambda) \mathcal{W}_{j}(\lambda)$ (see (2.17) $)$ shows that

$$
\mathcal{W}_{[0, j]}(\lambda)\left(\begin{array}{l}
1 \\
0
\end{array}\right)=\mathcal{W}_{[0, j-1]}(\lambda)\left(\begin{array}{c}
0 \\
\varepsilon_{j} b_{j}
\end{array}\right)=\left(\begin{array}{c}
-\varepsilon_{j} b_{j} Q_{j}(\lambda) \\
\varepsilon_{j} b_{j} P_{j}(\lambda)
\end{array}\right), \quad j \in \mathbb{N} .
$$

Therefore, the matrix $\mathcal{W}_{[0, j]}(\lambda)$ has the form

$$
\mathcal{W}_{[0, j]}(\lambda)=\left(\begin{array}{rr}
-\varepsilon_{j} b_{j} Q_{j}(\lambda) & -Q_{j+1}(\lambda) \\
\varepsilon_{j} b_{j} P_{j}(\lambda) & P_{j+1}(\lambda)
\end{array}\right), \quad j \in \mathbb{Z}_{+} .
$$

Next, writing the identity

$$
\left(\begin{array}{r}
-Q_{j+1}(\lambda) \\
P_{j+1}(\lambda)
\end{array}\right)=\mathcal{W}_{[0, j-1]}(\lambda) \mathcal{W}_{j}(\lambda)\left(\begin{array}{l}
0 \\
1
\end{array}\right)=\frac{1}{b_{j}} \mathcal{W}_{[0, j-1]}(\lambda)\left(\begin{array}{c}
-\varepsilon_{j} \\
p_{j}(\lambda)
\end{array}\right), \quad j \in \mathbb{N},
$$

componentwise, we see that the polynomials $P_{j}(\lambda)$ and $Q_{j}(\lambda)$ are the solutions of the difference equation

$$
\varepsilon_{j-1} \varepsilon_{j} b_{j-1} u_{j-1}-p_{j}(\lambda) u_{j}+b_{j} u_{j+1}=0, \quad j \in \mathbb{N},
$$

with the initial conditions

$$
\begin{aligned}
& P_{0}(\lambda)=1, \quad P_{1}(\lambda)=\frac{p_{0}(\lambda)}{b_{0}}, \\
& Q_{0}(\lambda)=0, \quad Q_{1}(\lambda)=\frac{\varepsilon_{0}}{b_{0}} .
\end{aligned}
$$

Therefore, we have the following result. 
Proposition 2.4. Let $P_{j}(\lambda)$ and $Q_{j}(\lambda)$ be the solutions of the difference equation (2.23) satisfying the initial conditions (2.24). Then the matrix of solutions of the problem $M_{\kappa}\left(\mathbf{s}, n_{N+1}-1\right)$ has the form

$$
\mathcal{W}_{[0, N]}(\lambda)=\left(\begin{array}{rr}
-\varepsilon_{N} b_{N} Q_{N}(\lambda) & -Q_{N+1}(\lambda) \\
\varepsilon_{N} b_{N} P_{N}(\lambda) & P_{N+1}(\lambda)
\end{array}\right)
$$

Applying consecutively fractional linear transformations $T_{j}\left(j \in \mathbb{Z}_{+}\right)$we get a continuous $P$-fraction (1.18). Formulas (2.20), (2.17) and (2.15) imply that the fraction $-\frac{P_{j+1}(\lambda)}{Q_{j+1}(\lambda)}$ is a partial fraction of the continuous fraction (1.18). In 20], to a $P$-fraction (1.18) the author associated a generalized Jacobi matrix uniquely determined by the parameters $\varepsilon_{j}, b_{j}, p_{j}$ of this fraction.

Remark 2.5. The algorithm described in 2.3 was first suggested in [19. In [3, 4] a similar algorithm was used to solve the factorization problem for $J$-unitary matrix polynomials.

\section{Generalized Jacobi matrices and the corresponding Weyl functions}

3.1. Definitions. Let $p(\lambda)=p_{k} \lambda^{k}+\cdots+p_{1} \lambda+p_{0}$ be a normalized (i.e., $p_{k}=1$ ) real polynomial of degree $k$. To the polynomial $p$ we associate its symmetrizer $E_{p}$ and the associated matrix $C_{p}$ given by

$$
E_{p}=\left(\begin{array}{ccc}
p_{1} & \ldots & p_{k} \\
\vdots & . \cdot & \\
p_{k} & & \mathbf{0}
\end{array}\right), \quad C_{p}=\left(\begin{array}{cccc}
0 & \ldots & 0 & -p_{0} \\
1 & & \mathbf{0} & -p_{1} \\
& \ddots & & \vdots \\
\mathbf{0} & & 1 & -p_{k-1}
\end{array}\right) .
$$

It is known that $\operatorname{det}\left(\lambda-C_{p}\right)=p(\lambda)$, the spectrum $\sigma\left(C_{p}\right)$ of $C_{p}$ is simple, and $E_{p}$ and $C_{p}$ are related by the equality (see [15])

$$
C_{p} E_{p}=E_{p} C_{p}^{\top} .
$$

Therefore, the matrix $C_{p} E_{p}$ is symmetric. One can easily see that $E_{p}^{-1}$ is also a Hankel matrix and has the form

$$
E_{p}^{-1}=\left(\begin{array}{ccc}
\mathbf{0} & & g_{k} \\
& . \cdot & \vdots \\
g_{k} & \ldots & g_{2 k}
\end{array}\right), \quad g_{k}=1
$$

Definition 3.1 ([34, 20]). Let $p_{j}$ be normalized real polynomials of degree $k_{j}$,

$$
p_{j}(\lambda)=\lambda^{k_{j}}+p_{k_{j}-1}^{(j)} \lambda^{k_{j}-1}+\cdots+p_{1}^{(j)} \lambda+p_{0}^{(j)},
$$

and let $\varepsilon_{j}= \pm 1, b_{j}>0(j \in \mathbb{N})$. The generalized Jacobi matrix associated with the sequence of polynomials $\left\{\varepsilon_{j} p_{j}\right\}_{j=0}^{\infty}$ and numbers $\left\{b_{j}\right\}_{j=0}^{\infty}$ is the tridiagonal block matrix

$$
H=\left(\begin{array}{cccc}
A_{0} & \widetilde{B}_{0} & & \mathbf{0} \\
B_{0} & A_{1} & \widetilde{B}_{1} & \\
& B_{1} & A_{2} & \ddots \\
\mathbf{0} & & \ddots & \ddots
\end{array}\right),
$$

where $A_{j}=C_{p_{j}}$ and the $k_{j+1} \times k_{j}$-matrix $B_{j}$ and the $k_{j} \times k_{j+1}$-matrix $\widetilde{B}_{j}$ are given by the formulas

$$
B_{j}=\left(\begin{array}{ccc}
0 & \ldots & b_{j} \\
\ldots & \ldots & \ldots \\
0 & \ldots & 0
\end{array}\right), \quad \widetilde{B}_{j}=\left(\begin{array}{ccc}
0 & \ldots & \widetilde{b}_{j} \\
\ldots & \ldots & \ldots \\
0 & \ldots & 0
\end{array}\right) \quad\left(\widetilde{b}_{j}=\varepsilon_{j} \varepsilon_{j+1} b_{j}, j=0, \ldots, N-1\right)
$$


Remark 3.2. Let us note that the columns and the rows of the matrix $H$ are numbered starting with 0 . According to (2.14) the indices $\kappa_{-}\left(\varepsilon_{j} p_{j}\right)$ occurring in the process described in 2.3 satisfy the condition

$$
\kappa_{-}\left(\varepsilon_{j} p_{j}\right)=\kappa_{j}-\kappa_{j-1},
$$

where $\kappa_{j}=$ sq_ $S_{n_{j+1}-1}$. Since for sufficiently large $j(\geq N)$ the indices $\kappa_{j}$ become equal to $\kappa$, the degrees of the polynomials $p_{j}$ equal 1 and $\varepsilon_{j}=1$ for $j \geq N$. In the sequel we will consider generalized Jacobi matrices of this form.

By $\ell_{[0, \infty)}^{2}$ we will denote the Hilbert space of square-summable complex sequences $\left(w_{0}, w_{1}, \ldots\right), w_{i} \in \mathbb{C}$. Let

$$
n_{0}=0, \quad n_{j}=\sum_{i=0}^{j-1} k_{i}, \quad j \in \mathbb{N} .
$$

Define the standard basis in $\ell_{[0, \infty)}^{2}$ by the formula

$$
e_{j, k}=\left\{\delta_{l, n_{j}+k}\right\}_{l=0}^{\infty} \quad\left(j \in \mathbb{Z}_{+} ; k=0, \ldots, k_{j}-1\right), \quad e:=e_{0,0} .
$$

Define an infinite symmetric matrix $G$ by the formula

$$
G=\operatorname{diag}\left(G_{0}, G_{1}, \ldots\right), \quad G_{j}=\varepsilon_{j} E_{p_{j}}^{-1} \quad\left(j \in \mathbb{Z}_{+}\right) .
$$

Let us note that in our case elements of the matrix $G$ are uniformly bounded since for a sufficiently large $j$ the matrix $G_{j}$ is one-dimensional and $G_{j}=1$.

Let $H_{[j, l]}\left(G_{[j, l]}\right)$ be the submatrix of the matrix $H(G)$ corresponding to the basis vectors $\left\{e_{i, k}\right\}_{k=0, \ldots, k_{i}-1}^{i=j, \ldots, l}(0 \leq j \leq l<+\infty)$. We will call $H_{[j, l]}$ a generalized Jacobi matrix.

3.2. Symmetric operators associated to a generalized Jacobi matrix. Let $\mathfrak{H}_{[j, l]}$ be the Pontryagin space $\mathbb{C}^{n_{l}-n_{j-1}}$ with the indefinite inner product

$$
[x, y]_{[j, l]}=\left(G_{[j, l]} x, y\right)_{\mathfrak{H}_{[j, l]}} \quad\left(x, y \in \mathbb{C}^{n_{l}-n_{j-1}}\right) .
$$

Let $S_{[j, l]}$ and $A_{[j, l]}$ be symmetric operators in $\mathfrak{H}_{[j, l]}$ defined as the restrictions of the operator $H_{[j, l]}$ to the space of vectors in $\mathfrak{H}_{[j, l]}$ orthogonal to $e_{j, 0}$ and $e_{l, 0}$ respectively,

$$
\begin{aligned}
\operatorname{dom} S_{[j, l]} & =\left\{x \in \mathfrak{H}_{[j, l]}:\left[x, e_{j, 0}\right]=0\right\}, \\
\operatorname{dom} A_{[j, l]} & =\left\{x \in \mathfrak{H}_{[j, l]}:\left[x, e_{l, 0}\right]=0\right\} .
\end{aligned}
$$

The operators $S_{[j, l]}$ and $A_{[j, l]}$ are symmetric operators with nondense domain and the defect indices $(1,1)$.

In the infinite-dimensional case the linear operator associated with the matrix $G$ is constructed as follows: first, using ordinary matrix multiplication, we define the operator on the linear set $\mathfrak{C}_{0}$ of finite sequences, and then take its closure. The constructed operator will also be denoted by $G$. On the entire space $\ell_{[0, \infty)}^{2}$ the operator $G$ is bounded and selfadjoint. Moreover, the operator $G$ is uniformly invertible, i.e., $G^{-1}$ is a bounded linear operator in $\ell_{[0, \infty)}^{2}$. Let $\mathfrak{H}_{[0, \infty)}$ be the space $\ell_{[0, \infty)}^{2}$ with the following indefinite inner product:

$$
[x, y]=(G x, y)_{\ell_{[0, \infty)}^{2}} \quad\left(x, y \in \ell_{[0, \infty)}^{2}\right) .
$$

The space $\mathfrak{H}_{[0, \infty)}$ is a Pontryagin space with the negative index $\kappa:=$ sq_ $_{-} G$.

To the matrix $H$ one can associate the operator $A$ in the space $\mathfrak{H}_{[0, \infty)}$ defined on $\mathfrak{C}_{0}$ by the formula $A x=H x$. The operator has dense domain and is symmetric in $\mathfrak{H}_{[0, \infty)}$ (see [20]), i.e.,

$$
[A x, y]=[x, A y] \quad\left(x, y \in \mathfrak{C}_{0}\right) .
$$


Relations (3.10) mean that $A \subset A^{+}$, i.e., $\operatorname{dom} A \subset \operatorname{dom} A^{+}$and $A x=A^{+} x$ for $x \in \operatorname{dom} A$. It is clear that $A^{+}$is a closed operator, and the operator $A$ admits a closure in $\ell_{[0, \infty)}^{2}(G)$ denoted by $A_{\min }$.

Together with the minimal operator $A_{\min }$ we consider the maximal operator $A_{\max }$ with the domain

$$
\operatorname{dom} A_{\max }=\left\{x \in \ell_{[0, \infty)}^{2}(G): H x \in \ell_{[0,+\infty)}^{2}(G)\right\}
$$

acting by the formula $A_{\max } x:=H x, x \in \operatorname{dom} A_{\max }$. One can easily see that $A_{\max }=$ $A^{+}=A_{\min }^{+}$. In the case where the symmetric operator $A_{\min }$ is not selfadjoint, its defect subspaces can be determined explicitly in terms of polynomials of the first kind.

3.3. Polynomials of the first and the second kind. Setting $\widetilde{b}_{-1}=\varepsilon_{0}$ we define polynomials $P_{j}(\lambda)$ of the first kind (see [34]) associated with the matrix $H$ as the solutions $u_{j}=P_{j}(\lambda)$ of the system

$$
\widetilde{b}_{j-1} u_{j-1}-p_{j}(\lambda) u_{j}+b_{j} u_{j+1}=0 \quad\left(j \in \mathbb{Z}_{+}\right)
$$

satisfying the boundary conditions

$$
u_{-1}=0, \quad u_{0}=1
$$

Similarly, polynomials of the second kind $Q_{j}(\lambda)$ associated with the matrix $H$ are defined as the solutions $u_{j}=Q_{j}(\lambda)$ of the system (3.11) satisfying the boundary conditions

$$
u_{-1}=-1, \quad u_{0}=0 .
$$

Formula (3.11) implies that the polynomials $P_{j}$ and $Q_{j}$ are of degrees $n_{j}$ and $n_{j}-k_{0}$ with leading coefficients $\left(b_{0} \ldots b_{j-1}\right)^{-1}$ and $\varepsilon_{0}\left(b_{0} \ldots b_{j-1}\right)^{-1}$ respectively. In particular,

$$
P_{1}(\lambda)=\frac{p_{0}(\lambda)}{b_{0}}, \quad Q_{0}(\lambda)=\frac{\varepsilon_{0}}{b_{0}} .
$$

Let us note that the difference equation (3.11) coincides with the three-term recurrence relation associated with the following $P$-fraction ([41], see also [26, Section 5.2]):

$$
-\frac{\varepsilon_{0}}{p_{0}(\lambda)}-\frac{\varepsilon_{0} \varepsilon_{1} b_{0}^{2}}{p_{1}(\lambda)}-\ldots \quad-\frac{\varepsilon_{j-1} \varepsilon_{j} b_{j-1}^{2}}{p_{j}(\lambda)}-\ldots .
$$

Moreover, polynomials of the first and second kind are just the polynomials associated with the $P$-fraction and satisfying the relation (2.23). Let us extend the systems of polynomials $\left\{P_{j}(\lambda)\right\}_{j=0}^{\infty},\left\{Q_{j}(\lambda)\right\}_{j=0}^{\infty}$ as follows:

$$
P_{j, k}(\lambda)=\lambda^{k} P_{j}(\lambda), \quad Q_{j, k}(\lambda)=\lambda^{k} Q_{j}(\lambda) \quad\left(j \in \mathbb{Z}_{+} ; k=0, \ldots, k_{j}-1\right)
$$

and set

$$
\begin{aligned}
& \mathbf{P}_{[l, j]}(\lambda)=\left(P_{l, 0}(\lambda), \ldots, P_{l, k_{l}-1}(\lambda), \ldots, P_{j, 0}(\lambda), \ldots, P_{j, k_{j}-1}(\lambda)\right), \\
& \mathbf{Q}_{[l, j]}(\lambda)=\left(Q_{l, 0}(\lambda), \ldots, Q_{l, k_{l}-1}(\lambda), \ldots, Q_{j, 0}(\lambda), \ldots, Q_{j, k_{j}-1}(\lambda)\right) .
\end{aligned}
$$

Then the system (3.11), (3.15) can be written in the following vector form:

$$
\begin{aligned}
& \mathbf{P}_{[0, j]}(\lambda)\left(\lambda-H_{[0, j]}\right)=\left(0, \ldots, 0, b_{j} P_{j+1,0}(\lambda)\right), \quad j \in \mathbb{Z}_{+}, \\
& \mathbf{Q}_{[0, j]}(\lambda)\left(\lambda-H_{[0, j]}\right)=(\underbrace{0, \ldots, 0,-\varepsilon_{0}}_{k_{0}}, 0, \ldots, 0, b_{j} Q_{j+1,0}(\lambda)), \quad j \in \mathbb{Z}_{+} .
\end{aligned}
$$

Removing the first $k_{0}$ columns of (3.17) and taking into account that

$$
Q_{0,0}(\lambda)=\cdots=Q_{0, k_{0}-1}(\lambda) \equiv 0,
$$

we obtain

$$
\mathbf{Q}_{[1, j]}(\lambda)\left(\lambda-H_{[1, j]}\right)=\left(0, \ldots, 0, b_{j} Q_{j+1,0}(\lambda)\right), \quad j \in \mathbb{N} .
$$


Formulas (3.16) and (3.18) imply that the eigenvalues of the matrices $H_{[0, j]}$ and $H_{[1, j]}$ coincide with the roots of the polynomials $P_{j+1}(\lambda), Q_{j+1}(\lambda)$. Taking into account the form of the leading coefficients of these polynomials we obtain the following formulas for the polynomials of the first and the second kind, which in the classical case can be found in [10, Section 7.1.2] and [5, Section 6.1].

Proposition 3.3 ([20]). The polynomials $P_{j}$ and $Q_{j}(j \in \mathbb{N})$ are computed by the formulas

$$
\begin{aligned}
P_{j}(\lambda) & =\left(b_{0} \ldots b_{j-1}\right)^{-1} \operatorname{det}\left(\lambda-H_{[0, j-1]}\right), \\
Q_{j}(\lambda) & =\varepsilon_{0}\left(b_{0} \ldots b_{j-1}\right)^{-1} \operatorname{det}\left(\lambda-H_{[1, j-1]}\right) .
\end{aligned}
$$

Proposition 3.4. The polynomials $P_{j}$ and $P_{j+1}\left(Q_{j}\right.$ and $\left.Q_{j+1}\right)$ do not have common roots.

Proof. Let us assume that the polynomials $P_{j}$ and $P_{j+1}$ have a common root $\lambda_{0}$, i.e., $P_{j}\left(\lambda_{0}\right)=P_{j+1}\left(\lambda_{0}\right)=0$. By (3.11), we have $P_{i}\left(\lambda_{0}\right)=0(i=0, \ldots, j+1)$. However, this is impossible because of the initial conditions (3.15). Similarly one proves that $Q_{j}$ and $Q_{j+1}$ do not have common roots.

Remark 3.5. The absence of common roots of the polynomials $P_{j}$ and $Q_{j}$ was established in 20 .

Taking into account the inequality

$$
G_{[0, j]} H_{[0, j]}=H_{[0, j]}^{\top} G_{[0, j]}
$$

and setting

$$
\pi_{[0, j]}(\lambda)=G_{[0, j]}^{-1} \mathbf{P}_{[0, j]}(\lambda)^{\top}, \quad \xi_{[0, j]}(\lambda)=G_{[0, j]}^{-1} \mathbf{Q}_{[0, j]}(\lambda)^{\top},
$$

we can rewrite (3.16), (3.17) in the form

$$
\begin{aligned}
\left(\lambda-H_{[0, j]}\right) \pi_{[0, j]}(\lambda) & =\varepsilon_{j} b_{j} P_{j+1,0}(\lambda) e_{j, 0}, \quad j \in \mathbb{Z}_{+}, \\
\left(\lambda-H_{[0, j]}\right) \xi_{[0, j]}(\lambda)+e_{0,0} & =\varepsilon_{j} b_{j} Q_{j+1,0}(\lambda) e_{j, 0}, \quad j \in \mathbb{Z}_{+} .
\end{aligned}
$$

Equation (3.21) implies that the defect subspace $\mathfrak{N}_{\lambda}\left(A_{[0, j]}\right)$ of the operator $A_{[0, j]}$ is given by the formula

$$
\mathfrak{N}_{\lambda}\left(A_{[0, j]}\right)=\operatorname{span}\left\{\pi_{[0, j]}(\lambda)\right\}, \quad \lambda \in \mathbb{C},
$$

since for all $h \in \operatorname{dom} A_{[0, j]}$ formula (3.21) implies that

$$
\left[\pi_{[0, j]}(\lambda),\left(A_{[0, j]}-\bar{\lambda}\right) h\right]=\left[\left(H_{[0, j]}-\lambda\right) \pi_{[0, j]}(\lambda), h\right]=0 .
$$

Now define

$$
\begin{aligned}
& \pi(\lambda)=G^{-1}\left(P_{0,0}(\lambda), \ldots, P_{0, k_{0}-1}(\lambda), \ldots\right)^{\top}, \\
& \xi(\lambda)=G^{-1}\left(Q_{0,0}(\lambda), \ldots, Q_{0, k_{0}-1}(\lambda), \ldots\right)^{\top} .
\end{aligned}
$$

One can easily establish the following formal equalities:

$$
\left(\lambda-A^{+}\right) \pi(\lambda)=0, \quad\left(\lambda-A^{+}\right) \xi(\lambda)=-e_{0,0} .
$$

Relation (3.24) shows that the equation

$$
\left(\lambda-A^{+}\right) g=0
$$

has a nontrivial solution if and only if $\pi(\lambda) \in \ell_{[0, \infty)}^{2}(G)$, and if this condition is satisfied, then the defect subspace $\mathfrak{N}_{\lambda}\left(A_{\text {min }}\right)$ of the operator $A_{\text {min }}$ is given by

$$
\mathfrak{N}_{\lambda}\left(A_{\min }\right)=\operatorname{span}\{\pi(\lambda)\}, \quad \lambda \in \mathbb{C} .
$$


Since the polynomials $P_{j k}(\lambda)$ are real, we have $n_{+}\left(A_{\min }\right)=n_{-}\left(A_{\min }\right)$. Therefore, the defect indices of the operator $A_{\min }$ are either $(0,0)$ or $(1,1)$. Therefore, we have the following result.

Proposition 3.6. The operator $A_{\min }$ is selfadjoint if and only if

$$
\sum_{j=0}^{\infty}\left|P_{j 0}(\lambda)\right|^{2}=\infty \quad \text { for some } \lambda \in \mathbb{C} .
$$

On the other hand, if the series in (3.27) converges for all $\lambda \in \mathbb{C}$, then the defect indices of the operator $A_{\min }$ are $(1,1)$ and the defect subspace $\mathfrak{N}_{\lambda}\left(A_{\min }\right)$ is generated by the vector $\pi(\lambda)$.

3.4. The boundary triple and the Weyl function of the operator $A_{[0, j]}$. Let us recall (see [6, 21]) that a linear relation in a Pontryagin space $\mathfrak{H}$ is a linear subspace in the space $\mathfrak{H} \oplus \mathfrak{H}$. Identifying a linear operator with its graph, we will assume that the set of linear operators is embedded in the set of linear relations. We will use the following notation:

$$
\begin{aligned}
& \operatorname{dom} T=\left\{f \in \mathfrak{H}:\left\{f, f^{\prime}\right\} \in T \text { for some } f^{\prime} \in \mathfrak{H}\right\}, \quad \operatorname{ker} T=\{f \in \mathfrak{H}:\{f, 0\} \in T\}, \\
& \operatorname{ran} T=\left\{f \in \mathfrak{H}:\left\{f^{\prime}, f\right\} \in T \text { for some } f^{\prime} \in \mathfrak{H}\right\}, \operatorname{mul} T=\{f \in \mathfrak{H}:\{0, f\} \in T\}
\end{aligned}
$$

for the domain, the kernel, the range, and the multivalue part of a linear relation $T$. The resolution set $\rho(T)$ of a linear relation $T$ is defined as the set of all $\lambda \in \mathbb{C}$ such that the linear relation

$$
(T-\lambda)^{-1}=\left\{\left\{f^{\prime}-\lambda f, f\right\}:\left\{f, f^{\prime}\right\} \in T\right\}
$$

is the graph of a linear operator in $\mathfrak{H}$, called the resolution.

To compute the Weyl function and the resolution matrix of a symmetric operator in a Pontryagin space ([21], see also 25]) we will systematically use the notion of a boundary triple. Let us give the definition in the case of a symmetric operator $A$ with defect indices $(1,1)$. In the case where the domain of $A$ is not dense, the conjugate linear relation $A^{+}$ is defined by the formula

$$
A^{+}=\left\{\left\{g, g^{\prime}\right\} \in \mathfrak{H}^{2}:[A f, g]=\left[f, g^{\prime}\right] \text { for all } f \in \operatorname{dom} A\right\} .
$$

A triple $\Pi=\left\{\mathbb{C}, \Gamma_{0}, \Gamma_{1}\right\}$, where $\Gamma_{0}, \Gamma_{1}$ are maps from $A^{+}$to $\mathbb{C}$, is called (see [17, 25, 21] ) a boundary triple of the linear relation $A^{+}$if $\operatorname{ran}\left\{\Gamma_{0}, \Gamma_{1}\right\}=\mathbb{C}^{2}$ and for all $\widehat{f}=\left\{f, f^{\prime}\right\}$, $\widehat{g}=\left\{g, g^{\prime}\right\} \in A^{+}$we have

$$
\left[f^{\prime}, g\right]-\left[f, g^{\prime}\right]=\Gamma_{1} \widehat{f} \overline{\Gamma_{0} \widehat{g}}-\Gamma_{0} \widehat{f} \overline{\Gamma_{1} \widehat{g}} .
$$

Formulas

$$
\widetilde{A}_{\theta}=\operatorname{ker}\left(\Gamma_{1}-\theta \Gamma_{0}\right), \quad \theta \in \mathbb{R}, \quad \widetilde{A}_{\infty}=\operatorname{ker} \Gamma_{0}
$$

give a bijection between the set of selfadjoint extensions $\widetilde{A}_{\theta}$ of the relation $A$ and the set of parameters $\theta \in \mathbb{R} \cup\{\infty\}$.

To each boundary triple $\Pi$ one associates a function $M(\lambda)$ called the Weyl function and defined by the formula (see [25, 21])

$$
M(\lambda) \Gamma_{0} \widehat{f}_{\lambda}=\Gamma_{1} \widehat{f}_{\lambda}, \quad \widehat{f}_{\lambda}=\{f, \lambda f\}, \quad f \in \mathfrak{N}_{\lambda} .
$$

In the case where $A$ is a simple symmetric operator in a Pontryagin space $\mathfrak{H}$, the Weyl function $M(\cdot)$ belongs to the space $\mathbf{N}_{\kappa}$, where $\kappa=$ ind $\mathbf{H}_{-} \mathfrak{H}$, and is the $Q$-function of the operator $A$ in the sense of the definitions in [30, 39].

Theorem 3.7 (31). Let $A$ be a simple symmetric operator in a Pontryagin space and let $M(\cdot)$ be its Weyl function corresponding to a boundary triple $\Pi$. Then the domain of holomorphy of $M$ coincides with the set of regular points of the extension $\widetilde{A}_{\infty}=\operatorname{ker} \Gamma_{0}$. 
Corollary 3.8. Under the hypotheses of Theorem 3.7 the spectrum of an extension $\widetilde{A}_{\theta}$ $(\theta \in \mathbb{R})$ lying in $\rho\left(\widetilde{A}_{\infty}\right)$ coincides with the set of zeros of the function $M(\lambda)-\theta$ in $\rho\left(\widetilde{A}_{\infty}\right)$.

Proof. Let $\Pi=\left\{\mathbb{C}, \Gamma_{0}, \Gamma_{1}\right\}$ be a boundary triple for $A^{+}$. One can easily see that $\widetilde{\Pi}=$ $\left\{\mathbb{C}, \widetilde{\Gamma}_{0}, \widetilde{\Gamma}_{1}\right\}=\left\{\mathbb{C}, \Gamma_{1}-\theta \Gamma_{0},-\Gamma_{0}\right\}$ is also a boundary triple for $A^{+}$and the corresponding Weyl function is $\widetilde{M}(\lambda)=-(M(\lambda)-\theta)^{-1}$. Since $\operatorname{ker} \widetilde{\Gamma}_{0}=\operatorname{ker}\left(\Gamma_{1}-\theta \Gamma_{0}\right)$, the corollary follows from Theorem 3.7 .

Let us compute the Weyl function of the symmetric operator $A_{[0, j]}$ introduced earlier. The conjugate linear relation $A_{[0, j]}^{+}$can be represented in the form

$$
A_{[0, j]}^{+}=\left\{\widehat{f}=\left\{f, H_{[0, j]} f+\widetilde{b}_{j} f_{j+1} e_{j, 0}\right\}: f \in \mathfrak{H}_{[0, j]}, f_{j+1} \in \mathbb{C}\right\} .
$$

Denote

$$
W_{j}[\widehat{f}, \widehat{g}]:=\widetilde{b}_{j} \varepsilon_{j}\left|\begin{array}{cc}
f_{j+1} & g_{j+1} \\
f_{j} & g_{j}
\end{array}\right|, \quad f_{j}:=f_{j, k_{j}-1} .
$$

Proposition 3.9. For all $\widehat{f}=\left\{f, f^{\prime}\right\}, \widehat{g}=\left\{g, g^{\prime}\right\} \in A_{[0, j]}^{+}$we have

$$
\left[f^{\prime}, g\right]-\left[f, g^{\prime}\right]=W_{j}[\widehat{f}, \bar{g}] \text {. }
$$

Proof. Indeed, for

$$
\widehat{f}=\left\{f, H_{[0, j]} f+\widetilde{b}_{j} f_{j+1} e_{j, 0}\right\}, \quad \widehat{g}=\left\{g, H_{[0, j]} g+\widetilde{b}_{j} g_{j+1} e_{j, 0}\right\}
$$

we have the equality

$$
\begin{aligned}
{\left[f^{\prime}, g\right]-\left[f, g^{\prime}\right] } & =\left[H_{[0, j]} f+\widetilde{b}_{j} f_{j+1} e_{j, 0}, g\right]-\left[f, H_{[0, j]} g+\widetilde{b}_{j} g_{j+1} e_{j, 0}\right] \\
& =\widetilde{b}_{j} \varepsilon_{j}\left(f_{j+1} \overline{g_{j}}-f_{j} \bar{g}_{j+1}\right)=W_{j}[\widehat{f}, \bar{g}],
\end{aligned}
$$

which proves (3.32).

Remark 3.10. Let $f \in \mathfrak{H}_{[0, l]}$. For $j<l$ consider the vector

$$
\widehat{f}^{(j)}=\left\{P_{j} f, P_{j} H_{[0, l]} f\right\},
$$

where $P_{j}$ is the orthogonal projection to $\mathfrak{H}_{[0, j]}$. Then $\widehat{f}^{(j)}$ admits the representation

$$
\widehat{f}^{(j)}=\left\{f_{[0, j]}, H_{[0, j]} f_{[0, j]}+\widetilde{b}_{j} f_{j+1} e_{j, 0}\right\} \in A_{[0, j]}^{+},
$$

where the component $f_{j+1}$ coincides with $f_{j+1, k_{j+1}-1}$. In particular, for the vectors

$$
\begin{aligned}
\widehat{\pi}_{[0, j]}(\lambda) & =\left\{\pi_{[0, j]}(\lambda), \lambda \pi_{[0, j]}(\lambda)\right\} \\
& =\left\{\pi_{[0, j]}(\lambda), H_{[0, j]} \pi_{[0, j]}(\lambda)+\varepsilon_{j} b_{j} P_{j+1,0}(\lambda) e_{j, 0}\right\}, \\
\widehat{\xi}_{[0, j]}(\lambda) & =\left\{\xi_{[0, j]}(\lambda), \lambda \xi_{[0, j]}(\lambda)+e\right\} \\
& =\left\{\xi_{[0, j]}(\lambda), H_{[0, j]} \xi_{[0, j]}(\lambda)+\varepsilon_{j} b_{j} Q_{j+1,0}(\lambda) e_{j, 0}\right\}
\end{aligned}
$$

we obtain

$$
\begin{aligned}
\left(\pi_{[0, j]}(\lambda)\right)_{j} & =\varepsilon_{j} P_{j, 0}(\lambda), \quad\left(\pi_{[0, j]}(\lambda)\right)_{j+1}=\varepsilon_{j+1} P_{j+1,0}(\lambda), \\
\left(\xi_{[0, j]}(\lambda)\right)_{j} & =\varepsilon_{j} Q_{j, 0}(\lambda), \quad\left(\xi_{[0, j]}(\lambda)\right)_{j+1}=\varepsilon_{j+1} Q_{j+1,0}(\lambda) .
\end{aligned}
$$

Proposition 3.11. A boundary triple of the linear relation $A_{[0, j]}^{+}$can be represented in the form

$$
\Gamma_{1} \widehat{f}=\varepsilon_{j+1} f_{j+1}, \quad \Gamma_{0} \widehat{f}=b_{j} f_{j}=\varepsilon_{j} b_{j}\left[f, e_{j, 0}\right]
$$


The corresponding Weyl function is

$$
M_{[0, j]}(\lambda)=\frac{P_{j+1}(\lambda)}{\varepsilon_{j} b_{j} P_{j}(\lambda)} .
$$

Proof. The fact that the maps (3.37) form a boundary triple for $A_{[0, j]}^{+}$follows from (3.32). Next we consider the defect vector $\pi_{[0, j]}(\lambda)$ of the operator $A_{[0, j]}$. Formula (3.21) implies that

$$
\begin{aligned}
\widehat{\pi}_{[0, j]}(\lambda) & :=\left\{\pi_{[0, j]}(\lambda), \lambda \pi_{[0, j]}(\lambda)\right\} \\
& =\left\{\pi_{[0, j]}(\lambda), H_{[0, j]} \pi_{[0, j]}(\lambda)+\varepsilon_{j} b_{j} P_{j+1,0}(\lambda) e_{j, 0}\right\} \in A_{[0, j]}^{+} .
\end{aligned}
$$

Applying the operator (3.37) and taking into account (3.35), we get the formulas

$$
\Gamma_{1} \widehat{\pi}_{[0, j]}(\lambda)=P_{j+1,0}(\lambda), \quad \Gamma_{0} \widehat{\pi}_{[0, j]}(\lambda)=\varepsilon_{j} b_{j} P_{j, 0}(\lambda),
$$

which imply (3.38).

3.5. $m$-functions of generalized Jacobi matrices. Now we compute the Weyl function of the symmetric operator $S_{[0, j]}$ introduced earlier as the restriction of $H_{[0, j]}$ to

$$
\operatorname{dom} S_{[0, j]}=\left\{x \in \mathfrak{H}_{[0, j]}:[x, e]=0\right\} .
$$

The conjugate linear relation $S_{[0, j]}^{+}$is given by the formula

$$
S_{[0, j]}^{+}=\left\{\widehat{f}=\left\{f, H_{[0, j]} f+c e\right\}:[f, e]=0, c \in \mathbb{C}\right\} .
$$

Proposition 3.12. A boundary triple for $S_{[0, j]}^{+}$can be given in the form

$$
\Gamma_{0} \widehat{f}=-c, \quad \Gamma_{1} \widehat{f}=[f, e] .
$$

The corresponding Weyl function has the form

$$
m_{[0, j]}(\lambda)=\left[\left(H_{[0, j]}-\lambda\right)^{-1} e, e\right] .
$$

Proof. Indeed, for $\widehat{f}=\left\{f, H_{[0, j]} f+c e\right\}$ and $\widehat{g}=\left\{g, H_{[0, j]} g+d e\right\}$ we have

$$
\left[f^{\prime}, g\right]-\left[f, g^{\prime}\right]=\left[H_{[0, j]} f+c e, g\right]-\left[f, H_{[0, j]} g+d e\right]=c \overline{[g, e]}-[f, e] \bar{d} .
$$

The defect subspace $\mathfrak{N}_{\lambda}\left(S_{[0, j]}\right)$ of the operator $S_{[0, j]}$ is given the formula

$$
\mathfrak{N}_{\lambda}\left(S_{[0, j]}\right)=\operatorname{span}\left\{\left(H_{[0, j]}-\lambda\right)^{-1} e\right\} .
$$

The equality

$$
\widehat{f}_{\lambda}=\left\{\left(H_{[0, j]}-\lambda\right)^{-1} e, \lambda\left(H_{[0, j]}-\lambda\right)^{-1} e\right\}=\left\{\left(H_{[0, j]}-\lambda\right)^{-1} e, H_{[0, j]}\left(H_{[0, j]}-\lambda\right)^{-1} e-e\right\}
$$

and formulas (3.41) imply that

$$
\Gamma_{0} \widehat{f}_{\lambda}=1, \quad \Gamma_{1} \widehat{f}_{\lambda}=\left[\left(H_{[0, j]}-\lambda\right)^{-1} e, e\right] .
$$

This proves formula (3.42).

The function $m_{[0, j]}(\lambda)$ is also called the $m$-function of the generalized Jacobi matrix (see [13, 20]). The form (3.4) of the matrix $H$ implies that

$$
m_{[0, j]}(\lambda)=-\varepsilon_{0} \frac{\operatorname{det}\left(\lambda-H_{[1, j]}\right)}{\operatorname{det}\left(\lambda-H_{[0, j]}\right)} .
$$

Taking into account (3.19) and (3.20) we obtain the following representation for the function $m_{[0, j]}(\lambda)$ :

$$
m_{[0, j]}(\lambda)=-\frac{Q_{j+1}(\lambda)}{P_{j+1}(\lambda)} .
$$


3.6. The resolution matrix of the operator $A_{[0, j]}$. Representation (3.42) of the $m$ function $m_{[0, j]}(\lambda)$ yields the asymptotic expansion

$$
m_{[0, j]}(\lambda)=-\frac{s_{0}}{\lambda}-\frac{s_{1}}{\lambda^{2}}-\cdots-\frac{s_{2 n_{j+1}-2}}{\lambda^{2 n_{j+1}-1}}+o\left(\frac{1}{\lambda^{2 n_{j+1}-1}}\right) \quad(\lambda \widehat{\rightarrow} \infty),
$$

where the moments $s_{i}$ are given by

$$
s_{i}=\left[H_{[0, j]}^{i} e, e\right], \quad i \leq 2 n_{j+1}-2 .
$$

Therefore, to each generalized Jacobi matrix $H_{[0, j]}$ we associate the truncated indefinite moment problem $M_{\kappa}\left(\mathbf{s}, n_{j+1}-1\right)$ defined by (3.46) $)$, and the function $m_{[0, j]}(\lambda)$ is one of the solutions of this problem. Theorem 2.3 implies that all solutions of this problem for $\kappa=\kappa_{j}=$ sq_$_{-} S_{n_{j+1}-1}$ are given by formula (2.19) where $\tau$ is an arbitrary function in the class $N_{0}$ satisfying the Nevanlinna condition; the function $\tau \equiv 0$ gives the solution $\varphi(\lambda)=m_{[0, j]}(\lambda)$.

The operator approach to the moment problem $M\left(\mathbf{s}, n_{j+1}-1, \kappa\right)$ leads to another formula related to the notion of the $u$-resolution of the operator $A_{[0, j]}$. Recall (see [33]) that the function

$$
\left[(\widetilde{A}-\lambda)^{-1} u, u\right], \quad u \in \mathfrak{H}_{[0, j]},
$$

is called the $u$-resolution of the operator $A_{[0, j]}$ with index $\widetilde{\kappa}-\kappa_{j}$ if $\widetilde{A}$ is a selfadjoint extension of the symmetric operator $A_{[0, j]}$ acting in a Pontryagin space $\widetilde{\mathfrak{H}}\left(\supset \mathfrak{H}_{[0, j]}\right)$ with negative index $\widetilde{\kappa}$ satisfying the minimality condition

$$
\widetilde{\mathfrak{H}}=\overline{\operatorname{span}}\left\{u,(\widetilde{A}-\lambda)^{-1} u: \lambda \in \mathbb{C}\right\} .
$$

Here $\kappa_{j}=\operatorname{ind}_{-} \mathfrak{H}_{[0, j]}$.

Theorem 3.13. Let $n=n_{j+1}-1$ and let the matrix $S_{n}=\left(s_{i+l}\right)_{i, l=0}^{n}$ be nondegenerate and have exactly $\kappa_{j}$ negative eigenvalues. Then the formula

$$
F(\lambda)=\left[(\widetilde{A}-\lambda)^{-1} u, u\right], \quad u=e_{0,0},
$$

establishes a one-to-one correspondence between the set of all solutions of the truncated indefinite moment problem $M_{\kappa}(\mathbf{s}, n)$ and the set of all u-resolutions of the symmetric operator $A_{[0, j]}$ of index $\kappa-\kappa_{j}$.

The description of $u$-resolutions of a symmetric operator was given in [29, 33] in the framework of the theory of the resolution matrix, which we will briefly present below.

Let $A$ be a symmetric operator in a Pontryagin space $\mathfrak{H}$ with defect indices $(1,1)$, and let us be given a scale $u \in \mathfrak{H}$. A point $\lambda \in \mathbb{C}$ is called (see [29, 33]) a u-regular point of the operator $A$ if $\lambda \in \widehat{\rho}(A)$ and

$$
\mathfrak{H}=\operatorname{ran}(A-\lambda) \dot{+} \operatorname{span}\{u\} .
$$

Denote by $\rho(A, u)$ the set of $u$-regular points of the operator $A$. Define two functionals $\mathcal{P}(\lambda), \mathcal{Q}(\lambda): \mathfrak{H} \rightarrow \mathbb{C}$ holomorphic in $\rho(A, u)$ by the formulas

$$
f-(\mathcal{P}(\lambda) f) u \in \operatorname{ran}(A-\lambda), \quad \mathcal{Q}(\lambda) f=\left[(A-\lambda)^{-1}(f-(\mathcal{P}(\lambda) f) u), u\right] .
$$

Define also two vector-valued functions $\mathcal{P}(\lambda)^{+}$and $\mathcal{Q}(\lambda)^{+}$with values in $\mathfrak{H}$ by setting

$$
\left[\mathcal{P}(\lambda)^{+}, f\right]:=\overline{\mathcal{P}(\lambda) f}, \quad\left[\mathcal{Q}(\lambda)^{+}, f\right]:=\overline{\mathcal{Q}(\lambda) f}
$$

Direct verification shows that for all $\lambda \in \rho(A, u)$ we have

$$
\widehat{\mathcal{P}}(\lambda)^{+}:=\left\{\mathcal{P}(\lambda)^{+}, \bar{\lambda} \mathcal{P}(\lambda)^{+}\right\} \in A^{+}, \quad \widehat{\mathcal{Q}}(\lambda)^{+}:=\left\{\mathcal{Q}(\lambda)^{+}, \bar{\lambda} \mathcal{Q}(\lambda)^{+}+u\right\} \in A^{+} .
$$

The description of $u$-resolutions of a densely defined symmetric operator in a Hilbert space with equal finite defect indices was obtained by M. G. Krein in [29], and for densely defined operators in a Pontryagin space, by M. G. Krein and H. Langer in 33. For a 
nondensely defined symmetric operator in a Pontryagin space with the defect indices $(1,1)$ this description takes the following form (see [21, Theorem 5.2]).

Theorem 3.14. Let $A$ be a symmetric operator with defect indices $(1,1)$ in a Pontryagin space $\mathfrak{H}$ with the negative index $\kappa$, and let $u \in \mathfrak{H}$. Then there exists a matrix-valued function $\mathcal{W}(\lambda)=\left(w_{i, j}(\lambda)\right)_{i, j=1}^{2}$ holomorphic in $\rho(A, u)$ such that the formula

$$
\left[(\widetilde{A}-\lambda)^{-1} u, u\right]=T_{\mathcal{W}}[\tau(\lambda)]:=\frac{w_{11}(\lambda) \tau(\lambda)+w_{12}(\lambda)}{w_{21}(\lambda) \tau(\lambda)+w_{22}(\lambda)}, \quad \lambda \in \rho(A, u) \cap \rho(\widetilde{A}),
$$

establishes a one-to-one correspondence between the set of u-resolutions of index $\widetilde{\kappa}-\kappa$ of the operator $A$ and the set of functions $\tau \in N_{\widetilde{\kappa}-\kappa}$ such that

$$
w_{21}(\lambda) \tau(\lambda)+w_{22}(\lambda) \not \equiv 0 .
$$

If $\Pi=\left\{\mathbb{C}, \Gamma_{0}, \Gamma_{1}\right\}$ is a boundary triple for $A^{+}$, then the matrix $\mathcal{W}(\lambda)$ can be found by the formula

$$
\mathcal{W}(\lambda)=\left(\begin{array}{ll}
-\Gamma_{0} \widehat{\mathcal{Q}}(\lambda)^{+} & \Gamma_{0} \widehat{\mathcal{P}}(\lambda)^{+} \\
-\Gamma_{1} \widehat{\mathcal{Q}}(\lambda)^{+} & \Gamma_{1} \widehat{\mathcal{P}}(\lambda)^{+}
\end{array}\right)^{*}, \quad \lambda \in \rho(A, u) .
$$

The matrix $\mathcal{W}(\lambda)$ in formula (3.54) is called the u-resolution matrix of the operator $A$ corresponding to the boundary triple $\Pi$ and the scale $u$. Formula (3.28) implies (see 21] for details) that it satisfies the following identity:

$$
J-\mathcal{W}(\lambda) \mathcal{H}(\omega)^{*}=-i(\lambda-\bar{\omega}) \mathcal{G}(\lambda) \mathcal{G}(\omega)^{*},
$$

where

$$
J=\left(\begin{array}{rr}
0 & -i \\
i & 0
\end{array}\right), \quad \mathcal{G}(\lambda)=\left(\begin{array}{r}
-\mathcal{Q}(\lambda) \\
\mathcal{P}(\lambda)
\end{array}\right) .
$$

Formula (3.55) implies the following properties of the matrix $\mathcal{W}(\lambda)$ :

1) the kernel $K_{\omega}(\lambda)=\frac{J-\mathcal{W}(\lambda) J \mathcal{W}(\omega)^{*}}{-i(\lambda-\bar{\omega})}$ has $\kappa$ negative squares in $\rho(A, u)$;

2) for each $\lambda \in \rho(A, u)$ we have

$$
\mathcal{W}(\lambda) J \mathcal{W}(\bar{\lambda})^{*}=J
$$

In particular, the matrix $\mathcal{W}(\lambda)$ is $J$-unitary for all $\lambda \in \rho(A, u) \cap \mathbb{R}$, i.e.,

$$
\mathcal{W}(\lambda) J \mathcal{W}(\lambda)^{*}=J, \quad \lambda \in \rho(A, u) \cap \mathbb{R} .
$$

Now we compute all components $\rho(A, u), \mathcal{P}(\lambda), \mathcal{Q}(\lambda), \mathcal{W}(\lambda)$ of M. G. Krein's representation theory for the operator and the vector $u=e_{0,0}$.

Proposition 3.15. For the operator $A_{[0, j]}$ and the scale vector $u=e_{0,0}$, the set $\rho(A, u)$ coincides with $\mathbb{C}$ and the maps $\mathcal{P}(\lambda), \mathcal{Q}(\lambda): \mathfrak{H}_{[0, j]} \rightarrow \mathbb{C}$ are given by

$$
\mathcal{P}(\lambda) f=\left[f, \pi_{[0, j]}(\bar{\lambda})\right], \quad \mathcal{Q}(\lambda) f=\left[f, \xi_{[0, j]}(\bar{\lambda})\right] .
$$

Furthermore,

$$
\mathcal{P}(\lambda)^{+}=\pi_{[0, j]}(\bar{\lambda}), \quad \mathcal{Q}(\lambda)^{+}=\xi_{[0, j]}(\bar{\lambda}),
$$

and the vector functions $\widehat{\mathcal{P}}(\lambda)^{+}$and $\widehat{\mathcal{Q}}(\lambda)^{+}$coincide with the functions $\widehat{\pi}_{[0, j]}(\bar{\lambda})$ and $\widehat{\xi}_{[0, j]}(\bar{\lambda})$ from (3.33) and (3.34) respectively.

Proof. Let us assume that for some $\lambda \in \mathbb{C}$ the equation $\left(A_{[0, j]}-\lambda\right) h=0$ has a nontrivial solution $h \in \mathfrak{H}_{[0, j]}$. Then $\left(H_{[0, j]}-\lambda\right) h=0$ and formula (3.21) shows that $h=\pi_{[0, j]}(\lambda)$ and $P_{j+1}(\lambda)=0$. Moreover, formula $\left[h, e_{j, 0}\right]=0$ implies that

$$
\left[\pi_{[0, j]}(\lambda), e_{j, 0}\right]=\left(\mathbf{P}_{[0, j]}(\lambda)^{\top}, e_{j, 0}\right)=P_{j}(\lambda)=0 .
$$


But by Proposition 3.4 the polynomials $P_{j}(\lambda)$ and $P_{j+1}(\lambda)$ have no common roots. Therefore $\widehat{\rho}\left(A_{[0, j]}\right)=\mathbb{C}$.

Now let for some $h \in \operatorname{dom} A_{[0, j]}, c \in \mathbb{C}$,

$$
\left(A_{[0, j]}-\lambda\right) h=c u .
$$

Multiplying both sides by $\pi_{[0, j]}(\bar{\lambda})$ we obtain

$$
0=c\left[u, \pi_{[0, j]}(\bar{\lambda})\right]=c .
$$

Therefore, $\rho\left(A_{[0, j]}, u\right)=\mathbb{C}$. Formula

$$
f=\left(A_{[0, j]}-\lambda\right) h+(\mathcal{P}(\lambda) f) u
$$

implies that

$$
\left[f, \pi_{[0, j]}(\bar{\lambda})\right]=(\mathcal{P}(\lambda) f)\left[u, \pi_{[0, j]}(\bar{\lambda})\right]=\mathcal{P}(\lambda) f .
$$

Next, viewing $H_{[0, j]}$ as an extension of the operator $A_{[0, j]}$, we obtain from (3.50) that

$$
\mathcal{Q}(\lambda) f=\left[\left(A_{[0, j]}-\lambda\right)^{-1}(f-(\mathcal{P}(\lambda) f) u), u\right]=\left[f-(\mathcal{P}(\lambda) f) u,\left(H_{[0, j]}-\bar{\lambda}\right)^{-1} u\right] .
$$

Formulas (3.21) and (3.22) imply that

$$
\left(H_{[0, j]}-\bar{\lambda}\right)^{-1} u=\xi_{[0, j]}(\bar{\lambda})+\frac{Q_{j+1}(\bar{\lambda})}{P_{j+1}(\bar{\lambda})} \pi_{[0, j]}(\bar{\lambda}) .
$$

Substituting (3.59) in (3.58) and taking into account (3.23) we obtain

$$
\mathcal{Q}(\lambda) f=\left[f-(\mathcal{P}(\lambda) f) u, \xi_{[0, j]}(\bar{\lambda})\right]=\left[f, \xi_{[0, j]}(\bar{\lambda})\right] .
$$

Formula (3.57) immediately follows from (3.56).

Theorem 3.16. Let a boundary triple for $A_{[0, j]}^{+}$be represented in the form (3.37). Then the corresponding u-resolution matrix of the operator $A_{[0, j]}$ has the form

$$
\mathcal{W}_{[0, j]}(\lambda)=\left(\begin{array}{rr}
-\varepsilon_{j} b_{j} Q_{j}(\lambda) & -Q_{j+1}(\lambda) \\
\varepsilon_{j} b_{j} P_{j}(\lambda) & P_{j+1}(\lambda)
\end{array}\right) .
$$

Proof. Indeed, Proposition 3.15 implies that

$$
\widehat{\mathcal{P}}(\bar{\lambda})^{+}=\widehat{\pi}_{[0, j]}(\lambda)=\left\{\pi_{[0, j]}(\lambda), \lambda \pi_{[0, j]}(\lambda)\right\} .
$$

Taking into account (3.37) we obtain the bottom row of formula (3.60).

Now consider the vector

$$
\widehat{\mathcal{Q}}(\bar{\lambda})^{+}=\widehat{\xi}_{[0, j]}(\lambda)=\left\{\xi_{[0, j]}(\lambda), H_{[0, j]} \xi_{[0, j]}(\lambda)+b_{j} Q_{j+1,0}(\lambda) e_{j, 0}\right\} \in A_{[0, j]}^{+} .
$$

Applying operators $\Gamma_{0}$ and $\Gamma_{1}$ from (3.37) we obtain

$$
\Gamma_{1}\left(\widehat{\xi}_{[0, j]}(\lambda)\right)=Q_{j+1}(\lambda), \quad \Gamma_{0}\left(\widehat{\xi}_{[0, j]}(\lambda)\right)=\varepsilon_{j} b_{j} Q_{j}(\lambda),
$$

and (3.60) follows.

Equality (3.55) implies the following generalized Christoffel-Darboux formulas.

Corollary 3.17. For all $j \in \mathbb{Z}_{+}, \lambda \in \mathbb{C}$, and $\lambda_{0} \in \mathbb{R}$ we have

$$
\begin{aligned}
W_{j}\left[\xi(\lambda), \xi\left(\lambda_{0}\right)\right] & =\left(\lambda-\lambda_{0}\right)\left[\xi(\lambda), \xi\left(\lambda_{0}\right)\right]_{\mathfrak{H}_{[0, j]}}, \\
W_{j}\left[\xi(\lambda), \pi\left(\lambda_{0}\right)\right] & =1+\left(\lambda-\lambda_{0}\right)\left[\xi(\lambda), \pi\left(\lambda_{0}\right)\right]_{\mathfrak{H}_{[0, j]}}, \\
W_{j}\left[\pi(\lambda), \xi\left(\lambda_{0}\right)\right] & =-1+\left(\lambda-\lambda_{0}\right)\left[\pi(\lambda), \xi\left(\lambda_{0}\right)\right]_{\mathfrak{H}_{[0, j]}}, \\
W_{j}\left[\pi(\lambda), \pi\left(\lambda_{0}\right)\right] & =\left(\lambda-\lambda_{0}\right)\left[\pi(\lambda), \pi\left(\lambda_{0}\right)\right]_{\mathfrak{H}_{[0, j]}} .
\end{aligned}
$$


Proof. Rewriting (3.55) in the form (3.65)

$$
\begin{aligned}
& \mathcal{W}_{[0, j]}\left(\lambda_{0}\right)\left(\begin{array}{rr}
0 & 1 \\
-1 & 0
\end{array}\right) \mathcal{W}_{[0, j]}(\bar{\lambda})^{*} \\
& \quad=\left(\begin{array}{rr}
0 & 1 \\
-1 & 0
\end{array}\right)+\left(\lambda-\lambda_{0}\right)\left(\begin{array}{lc}
{\left[\mathcal{Q}(\bar{\lambda})^{+}, \mathcal{Q}\left(\lambda_{0}\right)^{+}\right]_{\mathfrak{H}_{[0, j]}}} & -\left[\mathcal{P}(\bar{\lambda})^{+}, \mathcal{Q}\left(\lambda_{0}\right)^{+}\right]_{\mathfrak{H}_{[0, j]}} \\
-\left[\mathcal{Q}(\bar{\lambda})^{+}, \mathcal{P}\left(\lambda_{0}\right)^{+}\right]_{\mathfrak{H}_{[0, j]}} & {\left[\mathcal{P}(\bar{\lambda})^{+}, \mathcal{P}\left(\lambda_{0}\right)^{+}\right]_{\mathfrak{H}_{[0, j]}}}
\end{array}\right),
\end{aligned}
$$

we obtain the following expression for the left-hand side of (3.65):

$$
\varepsilon_{j} b_{j}\left(\begin{array}{cc}
-\left(Q_{j+1}\left(\lambda_{0}\right) Q_{j}(\lambda)-Q_{j}\left(\lambda_{0}\right) Q_{j+1}(\lambda)\right) & \left(Q_{j+1}\left(\lambda_{0}\right) P_{j}(\lambda)-Q_{j}\left(\lambda_{0}\right) P_{j+1}(\lambda)\right) \\
\left(P_{j+1}\left(\lambda_{0}\right) Q_{j}(\lambda)-P_{j}\left(\lambda_{0}\right) Q_{j+1}(\lambda)\right) & -\left(P_{j+1}\left(\lambda_{0}\right) P_{j}(\lambda)-P_{j}\left(\lambda_{0}\right) P_{j+1}(\lambda)\right)
\end{array}\right) .
$$

Comparing matrix entries on the left- and right-hand sides of (3.65) and taking into account that

$$
\mathcal{P}(\bar{\lambda})^{+}=\pi_{[0, j]}(\lambda), \quad \mathcal{Q}(\bar{\lambda})^{+}=\xi_{[0, j]}(\lambda),
$$

we obtain formulas (3.61)-(3.64).

Setting $\lambda=\lambda_{0}$ in (3.62) we immediately obtain the generalized Liouville-Ostrogradskii formula.

Corollary 3.18. For each $j \in \mathbb{Z}_{+}$we have

$$
\varepsilon_{j} b_{j}\left(Q_{j+1}(\lambda) P_{j}(\lambda)-Q_{j}(\lambda) P_{j+1}(\lambda)\right)=1 .
$$

Theorem 3.16 and the factorization formula (2.17) imply the following result.

Corollary 3.19. The resolution matrix $\mathcal{W}_{[0, j]}(\lambda)$ admits the factorization

$$
\mathcal{W}_{[0, j]}(\lambda)=\mathcal{W}_{0}(\lambda) \ldots \mathcal{W}_{j}(\lambda)
$$

where $\mathcal{W}_{l}(\lambda)$ is of the form (2.16).

Let polynomials $P_{j}^{(l)}(\lambda)$ and $Q_{j}^{(l)}(\lambda)(j \geq l-1)$ be solutions of equation (3.11) with the initial conditions

$$
P_{l-1}^{(l)}=0, \quad P_{l}^{(l)}=1, \quad Q_{l-1}^{(l)}=-1, \quad Q_{l}^{(l)}=0,
$$

and let the vectors $\pi_{[l, j]}(\lambda)$ and $\xi_{[l, j]}(\lambda)$ be defined by the formulas

$$
\begin{aligned}
& \pi_{[l, j]}(\lambda)=G_{[l, j]}^{-1}\left(P_{l, 0}^{(l)}(\lambda), \ldots, P_{l, k_{l}-1}^{(l)}(\lambda), \ldots, P_{j, 0}^{(l)}(\lambda), \ldots, P_{j, k_{j}-1}^{(l)}(\lambda)\right)^{\top}, \\
& \xi_{[l, j]}(\lambda)=G_{[l, j]}^{-1}\left(Q_{l, 0}^{(l)}(\lambda), \ldots, Q_{l, k_{l}-1}^{(l)}(\lambda), \ldots, Q_{j, 0}^{(l)}(\lambda), \ldots, Q_{j, k_{j}-1}^{(l)}(\lambda)\right)^{\top} .
\end{aligned}
$$

Since the space $A_{[l, j]}^{+}$is of the form

$$
A_{[l, j]}^{+}=\left\{\widehat{f}=\left\{f, H_{[l, j]} f+\widetilde{b}_{j} f_{j+1} e_{j, 0}\right\}: f \in \mathfrak{H}_{[l, j]}, f_{j+1} \in \mathbb{C}\right\},
$$

the vectors

$$
\begin{aligned}
& \widehat{\pi}_{[l, j]}(\lambda)=\left\{\pi_{[l, j]}(\lambda), H_{[l, j]} \pi_{[l, j]}(\lambda)+\varepsilon_{j} b_{j} P_{j+1,0}^{(l)}(\lambda) e_{j, 0}\right\}, \\
& \widehat{\xi}_{[l, j]}(\lambda)=\left\{\xi_{[l, j]}(\lambda), H_{[l, j]} \xi_{[l, j]}(\lambda)+\varepsilon_{j} b_{j} Q_{j+1,0}^{(l)} e_{j, 0}\right\}
\end{aligned}
$$

belong to $A_{[l, j]}^{+}$. Defining $f_{j}$ and $f_{j+1}$ by formulas (3.31) and (3.68), we obtain

$$
\begin{aligned}
\left(\pi_{[l, j]}(\lambda)\right)_{j} & =\varepsilon_{j} P_{j, 0}^{(l)}(\lambda), \quad\left(\pi_{[l, j]}(\lambda)\right)_{j+1}=\varepsilon_{j+1} P_{j+1,0}^{(l)}(\lambda), \\
\left(\xi_{[l, j]}(\lambda)\right)_{j} & =\varepsilon_{j} Q_{j, 0}^{(l)}(\lambda), \quad\left(\xi_{[l, j]}(\lambda)\right)_{j+1}=\varepsilon_{j+1} Q_{j+1,0}^{(l)}(\lambda) .
\end{aligned}
$$


Together with (3.54), this formula implies that the $u$-resolution matrix of the operator $A_{[l, j]}$ corresponding to the scale vector $u=e_{l, 0}$ and the boundary triple (3.37) is of the form

$$
\mathcal{W}_{[l, j]}(\lambda)=\left(\begin{array}{rr}
-\varepsilon_{j} b_{j} Q_{j}^{(l)}(\lambda) & -Q_{j+1}^{(l)}(\lambda) \\
\varepsilon_{j} b_{j} P_{j}^{(l)}(\lambda) & P_{j+1}^{(l)}(\lambda)
\end{array}\right) .
$$

Applying the factorization (2.17) to the matrix $\mathcal{W}_{[l, j]}(\lambda)$ we obtain

$$
\mathcal{W}_{[l, j]}(\lambda)=\mathcal{W}_{l}(\lambda) \ldots \mathcal{W}_{j}(\lambda)
$$

From (2.17) and (3.74) we obtain the following result.

Theorem 3.20. Let $0 \leq l<j$. Then the resolution matrices $\mathcal{W}_{[l, j]}(\lambda)$ and $\mathcal{W}_{[0, j]}(\lambda)$ are related as follows:

$$
\mathcal{W}_{[0, j]}(\lambda)=\mathcal{W}_{[0, l-1]}(\lambda) \mathcal{W}_{[l, j]}(\lambda) .
$$

Let $H_{[0, j]}$ be a generalized Jacobi matrix, $n=n_{j+1}-1$, and suppose the corresponding sequence of moments $\mathbf{s}=\left(s_{i}\right)_{i=0}^{2 n}$ satisfies the condition sq_ $\left(s_{i+l}\right)_{i, l=0}^{n}=\kappa$. Let $N$ be such that the matrix $\left(s_{i+j}\right)_{i, j=0}^{n_{N}-1}$ has exactly $\kappa$ negative eigenvalues. Then for $j \geq N-1$, the $m$ function $m_{[0, j]}(\lambda)$ is a solution of the truncated indefinite moment problem $M\left(\mathbf{s}, n_{N}-1, \kappa\right)$ and, according to Theorem 2.3, admits a representation

$$
m_{[0, j]}(\lambda)=\frac{w_{11}(\lambda) \tau(\lambda)+w_{12}(\lambda)}{w_{21}(\lambda) \tau(\lambda)+w_{22}(\lambda)}
$$

where $\mathcal{W}(\lambda)=\left(w_{i, l}(\lambda)\right)_{i, l=1}^{2}=\mathcal{W}_{[0, N-1]}(\lambda)$ and $\tau$ is a function of class $\mathbf{N}_{0}$ satisfying the Nevanlinna condition (1.11). The next proposition specifies the value of the parameter $\tau$ in this representation.

Corollary 3.21. For each $j \geq N$ we have

$$
m_{[0, j]}(\lambda)=\frac{w_{11}(\lambda) m_{[N, j]}(\lambda)+w_{12}(\lambda)}{w_{21}(\lambda) m_{[N, j]}(\lambda)+w_{22}(\lambda)}
$$

where $\left(w_{i j}(\lambda)\right)_{i, j=1}^{2}:=\mathcal{W}_{[0, N-1]}(\lambda)$.

Proof. Indeed, by Theorem 3.20

$$
\left(\begin{array}{rr}
-\varepsilon_{j} b_{j} Q_{j}(\lambda) & -Q_{j+1}(\lambda) \\
\varepsilon_{j} b_{j} P_{j}(\lambda) & P_{j+1}(\lambda)
\end{array}\right)=\mathcal{W}_{[0, N-1]}(\lambda)\left(\begin{array}{rr}
-\varepsilon_{j} b_{j} Q_{j}^{(N)}(\lambda) & -Q_{j+1}^{(N)}(\lambda) \\
\varepsilon_{j} b_{j} P_{j}^{(N)}(\lambda) & P_{j+1}^{(N)}(\lambda)
\end{array}\right),
$$

so that

$$
\begin{aligned}
-Q_{j+1}(\lambda) & =w_{11}(\lambda)\left(-Q_{j+1}^{(N)}(\lambda)\right)+w_{12}(\lambda) P_{j+1}^{(N)}(\lambda), \\
P_{j+1}(\lambda) & =w_{21}(\lambda)\left(-Q_{j+1}^{(N)}(\lambda)\right)+w_{22}(\lambda) P_{j+1}^{(N)}(\lambda) .
\end{aligned}
$$

Taking into account the formulas (see (3.44))

$$
m_{[0, j]}(\lambda)=-\frac{Q_{j+1}(\lambda)}{P_{j+1}(\lambda)}, \quad m_{[N, j]}(\lambda)=-\frac{Q_{j+1}^{(N)}(\lambda)}{P_{j+1}^{(N)}(\lambda)},
$$

we obtain (3.76). Here $P_{j+1}^{(N)}(\lambda)$ and $Q_{j+1}^{(N)}(\lambda)$ are the polynomials of the first and second kind corresponding to the generalized Jacobi matrix $H_{[N, j]}$.

In the special case $N=1$ formula (3.76) coincides with the generalized Riccati equation. 
Corollary 3.22. The $m$-functions $m_{[0, j]}(\lambda)$ and $m_{[1, j]}(\lambda)$ are related by the formula

$$
m_{[0, j]}(\lambda)=\frac{-\varepsilon_{0}}{p_{0}(\lambda)+\varepsilon_{0} b_{0}^{2} m_{[1, j]}(\lambda)}, \quad j \in \mathbb{N} .
$$

A consecutive application of formula (3.77) yields the $P$-fraction (1.18) that occurs in the stepwise solution process of the moment problem $M_{\kappa}(\mathbf{s})$.

3.7. Characteristic properties of the $u$-resolution matrix of the operator $A_{[0, j]}$. Let $\mathcal{W}(\lambda)=\left(w_{i, j}(\lambda)\right)_{i, j=1}^{2}$ be the $u$-resolution matrix of the operator $A_{[0, j]}$ corresponding to the boundary triple (3.37) and the scale vector $u=e$. Then formula (3.60) implies the following properties of $\mathcal{W}(\lambda)$ :

(W1) $\mathcal{W}(\lambda)$ is a polynomial matrix-valued function such that

$$
\operatorname{deg} w_{11}<\operatorname{deg} w_{21}, \quad \operatorname{deg} w_{12}<\operatorname{deg} w_{22}
$$

(W2) $\mathcal{W}(\lambda)$ is $J$-unitary on the real line;

(W3) the leading coefficients of $w_{12}$ and $w_{22}$ have equal absolute value.

As follows from (3.54), the $u$-resolution matrix $\widetilde{\mathcal{W}}(\lambda)$ of the operator $A_{[0, j]}$ corresponding to an arbitrary boundary triple differs from $\mathcal{W}(\lambda)$ by a right $J$-unitary constant factor. Each $J$-unitary constant matrix can be written as

$$
V=\left(\begin{array}{ll}
\alpha & \beta \\
\gamma & \delta
\end{array}\right) e^{i \theta}, \quad \alpha, \beta, \gamma, \delta, \theta \in \mathbb{R}
$$

One can easily see that properties (W1)-(W3) are invariant under the multiplication from the right by a matrix of the form (3.79). In addition to (W1)-(W3), each matrix $\mathcal{W}(\lambda)$ of the form (3.60) also has the following properties:

(W4) $\mathcal{W}(\lambda)$ is real on the real line;

(W5) $\operatorname{deg} w_{11}<\operatorname{deg} w_{12}$, $\operatorname{deg} w_{21}<\operatorname{deg} w_{22}$;

(W6) the leading coefficient of the polynomial $w_{22}$ is positive.

Properties (W4)-(W6) are not invariant under right multiplication by a constant $J$ unitary matrix.

Let us note that by $J$-unitarity of the matrix $\mathcal{W}(\lambda)$ for $\lambda \in \mathbb{R}$, the first condition in (3.78) and in (W5) is a corollary of the second.

Let us show that properties (W1)-(W3) completely characterize $u$-resolution matrices of the operator $A_{[0, N]}$ corresponding to the scale vector $u=e$.

Theorem 3.23. A polynomial vector-valued function $\mathcal{W}(\lambda)=\left(w_{i j}(\lambda)\right)_{i, j=1}^{2}$ is a $u$ resolution matrix of an operator $A_{[0, N]}$ generated by a generalized Jacobi matrix and a scale vector $u=e$ if and only if it satisfies conditions (W1)-(W3).

Proof. It remains to prove the sufficiency of conditions (W1)-(W3).

Let a matrix $\mathcal{W}(\lambda)$ satisfies conditions (W1)-(W3). The $J$-unitarity of the matrix $\mathcal{W}(\lambda)$ for $\lambda \in \mathbb{R}$ is equivalent to the following formulas:

$$
\begin{aligned}
& w_{12}(\lambda) \overline{w_{22}(\lambda)}=\overline{w_{12}(\lambda)} w_{22}(\lambda), \\
& w_{11}(\lambda) \overline{w_{21}(\lambda)}=\overline{w_{11}(\lambda)} w_{21}(\lambda), \\
& w_{21}(\lambda) \overline{w_{22}(\lambda)}-\overline{w_{21}(\lambda)} w_{22}(\lambda)=0, \\
& w_{11}(\lambda) \overline{w_{22}(\lambda)}-w_{12}(\lambda) \overline{w_{21}(\lambda)}=1 .
\end{aligned}
$$

Formulas (3.80) and (3.82) imply that the rational functions

$$
\frac{w_{12}(\lambda)}{w_{22}(\lambda)}, \quad \frac{w_{21}(\lambda)}{w_{22}(\lambda)}
$$


are real on $\mathbb{R}$; in particular, the ratio of the leading coefficients $a_{21}$ and $a_{22}$ of the polynomials $w_{21}$ and $w_{22}$ is real.

Let us choose a $J$-unitary matrix $V_{1}$ of the form (3.79) in such way that the matrixvalued function

$$
\widetilde{\mathcal{W}}(\lambda)=\left(\widetilde{w}_{i j}(\lambda)\right)_{i, j=1}^{2}:=\mathcal{W}(\lambda) V_{1}=\left(\begin{array}{ll}
w_{11}(\lambda) \alpha+w_{12}(\lambda) \gamma & w_{11}(\lambda) \beta+w_{12}(\lambda) \delta \\
w_{21}(\lambda) \alpha+w_{22}(\lambda) \gamma & w_{21}(\lambda) \beta+w_{22}(\lambda) \delta
\end{array}\right) e^{i \theta}
$$

satisfies conditions (W5), (W6). This can always be done. For example, if $\operatorname{deg} w_{21}>$ $\operatorname{deg} w_{22}$, we can set

$$
V_{1}=\left(\begin{array}{cc}
0 & \bar{a}_{21} \\
-1 / a_{21} & 0
\end{array}\right)
$$

If $\operatorname{deg} w_{21}=\operatorname{deg} w_{22}$, then the matrix $V_{1}$ should be chosen in the form

$$
V_{1}=\left(\begin{array}{cc}
1 / a_{22} & 0 \\
-a_{21} / a_{22}^{2} & \bar{a}_{22}
\end{array}\right)
$$

since the ratio $a_{21} / a_{22}$ is real, the matrix $V_{1}$ is of the form (3.79). Finally, if $\operatorname{deg} w_{21}<$ $\operatorname{deg} w_{22}$, then condition (W5) is already satisfied and in order for $\widetilde{\mathcal{W}}$ to satisfy condition (W6) it is sufficient to set

$$
V_{1}=\left(\begin{array}{cc}
1 / a_{22} & 0 \\
0 & \bar{a}_{22}
\end{array}\right) .
$$

Therefore, in addition to conditions (W1)-(W3) the entries $\widetilde{w}_{i j}(\lambda)$ satisfy conditions (W5), (W6). Formulas (3.83) and (3.80) imply that the polynomials $\widetilde{w}_{12}(\lambda)$ and $\widetilde{w}_{22}(\lambda)$ are relatively prime and the rational function $\varphi(\lambda)=\widetilde{w}_{12}(\lambda) / \widetilde{w}_{22}(\lambda)$ is real. Using condition (W5) we see that $\varphi \in \mathbf{N}_{\kappa,-\infty}$ for some $\kappa \in \mathbb{Z}_{+}$and $\varphi$ admits an expansion in a $P$-fraction

$$
\varphi(\lambda)=\frac{\widetilde{w}_{12}(\lambda)}{\widetilde{w}_{22}(\lambda)}=-\frac{\varepsilon_{0}}{p_{0}(\lambda)}-\frac{\varepsilon_{0} \varepsilon_{1} b_{0}^{2}}{p_{1}(\lambda)}-\ldots \quad-\frac{\varepsilon_{N-1} \varepsilon_{N} b_{N-1}^{2}}{p_{N}(\lambda)}
$$

using the algorithm presented in 2.3. According to condition (W3), we have $\varepsilon_{0}= \pm 1$.

Let $H_{[0, N]}$ be a generalized Jacobi matrix corresponding to the $P$-fraction (3.85) and let $P_{j}, Q_{j}(0 \leq j \leq N+1)$ be the polynomials of the first and second kind defined by formulas (3.19) and (3.20) with $b_{N}=1$. Let us establish a relation between the matrix $\widetilde{\mathcal{W}}(\lambda)$ and the $u$-resolution matrix

$$
\mathcal{W}_{[0, N]}(\lambda)=\left(\begin{array}{cc}
-\varepsilon_{N} Q_{N}(\lambda) & -Q_{N+1}(\lambda) \\
\varepsilon_{N} P_{N}(\lambda) & P_{N+1}(\lambda)
\end{array}\right)
$$

corresponding to a boundary triple $\left\{\mathbb{C}, \Gamma_{0}, \Gamma_{1}\right\}$ of the form (3.37). Since the rational function $-Q_{N+1}(\lambda) / P_{N+1}(\lambda)$ has the same $P$-fraction expansion (3.85) as the function $\widetilde{w}_{12} / \widetilde{w}_{22}$, and since $\widetilde{w}_{22}$ satisfies condition (W6), there is $c>0$ such that

$$
-Q_{N+1}(\lambda)=c \widetilde{w}_{12}(\lambda), \quad P_{N+1}(\lambda)=c \widetilde{w}_{22}(\lambda) .
$$

Next, using formula (3.87), identity (3.83) for $\widetilde{w}_{i j}(\lambda)$, and the Liouville-Ostrogradskii identity

$$
\varepsilon_{N}\left(Q_{N+1}(\lambda) P_{N}(\lambda)-Q_{N}(\lambda) P_{N+1}(\lambda)\right) \equiv 1,
$$

we obtain

$$
\left(\widetilde{w}_{11}(\lambda)+c \varepsilon_{N} Q_{N}(\lambda)\right) \widetilde{w}_{22}(\lambda)-\left(\widetilde{w}_{21}(\lambda)-c \varepsilon_{N} P_{N}(\lambda)\right) \widetilde{w}_{12}(\lambda) \equiv 0 .
$$

Since $\widetilde{w}_{12}(\lambda)$ and $\widetilde{w}_{22}(\lambda)$ are relatively prime, the polynomial $\widetilde{w}_{21}(\lambda)-c \varepsilon_{N} P_{N}(\lambda)$ is divisible by $\widetilde{w}_{22}(\lambda)$, and since $\operatorname{deg}\left(\widetilde{w}_{21}-c \varepsilon_{N} P_{N}\right)<\operatorname{deg} \widetilde{w}_{22}$, the polynomial $\widetilde{w}_{21}(\lambda)-c \varepsilon_{N} P_{N}(\lambda)$ 
vanishes identically. Therefore, the same is true for the polynomial $\widetilde{w}_{11}(\lambda)+c \varepsilon_{N} Q_{N}(\lambda)$. Hence,

$$
-\varepsilon_{N} Q_{N}(\lambda)=\frac{1}{c} \widetilde{w}_{11}(\lambda), \quad \varepsilon_{N} P_{N}(\lambda)=\frac{1}{c} \widetilde{w}_{21}(\lambda)
$$

Setting

$$
V_{2}=\left(\begin{array}{cc}
1 / c & 0 \\
0 & c
\end{array}\right)
$$

and using (3.87), (3.88), and (3.84) we obtain

$$
\mathcal{W}_{[0, N]}(\lambda)=\widetilde{\mathcal{W}}(\lambda) V_{2}=\mathcal{W}(\lambda) V_{2} V_{1} .
$$

Formulas (3.54) and (3.89) imply that $\mathcal{W}(\lambda)$ is a $u$-resolution matrix of the operator $A_{[0, j]}$ corresponding to the scale vector $u=e$ and a boundary triple $\left\{\mathbb{C}, \widetilde{\Gamma}_{0}, \widetilde{\Gamma}_{1}\right\}$ of the form

$$
\left(\begin{array}{c}
\widetilde{\Gamma}_{0} \\
\widetilde{\Gamma}_{1}
\end{array}\right)=V_{2} V_{1}\left(\begin{array}{c}
\Gamma_{0} \\
\Gamma_{1}
\end{array}\right)
$$

Corollary 3.24. Every polynomial matrix satisfying conditions (W1)-(W3) admits a factorization of the form

$$
\mathcal{W}(\lambda)=\mathcal{W}_{0}(\lambda) \ldots \mathcal{W}_{N}(\lambda) V
$$

where $\mathcal{W}_{j}(\lambda)$ is a factor of the form (2.16) and $V$ is a constant $J$-unitary matrix.

Remark 3.25. In 3] it was shown that each $J$-unitary polynomial matrix on $\mathbb{R}$ admits a factorization of the form (3.90) with somewhat different elementary factors $\mathcal{W}_{j}(\lambda)$. One can easily see that the $J$-unitary polynomial matrix $\mathcal{W}(\lambda)$ on $\mathbb{R}$ satisfies conditions (W1) and (W3) after the left multiplication by an appropriate $J$-unitary constant factor. Therefore, Corollary 3.24 shows that $\mathcal{W}(\lambda)$ admits a factorization

$$
\mathcal{W}(\lambda)=V_{1} \mathcal{W}_{0}(\lambda) \ldots \mathcal{W}_{N}(\lambda) V_{2},
$$

where $\mathcal{W}_{j}(\lambda)$ is a factor of the form (2.16) and $V_{1}, V_{2}$ are $J$-unitary constant matrices.

3.8. Generalized Nevanlinna matrices. The generalized Liouville-Nevanlinna formula (3.66) allows us to rewrite the Green formula (3.32) as follows.

Proposition 3.26. For $\widehat{f}=\left\{f, f^{\prime}\right\}, \widehat{g}=\left\{g, g^{\prime}\right\} \in A_{[0, j]}^{+}$and $\lambda_{0} \in \mathbb{R}$ we have

$$
\left[f^{\prime}, g\right]-\left[f, g^{\prime}\right]=W_{j}\left[\widehat{f}, \widehat{\xi}_{[0, j]}\left(\lambda_{0}\right)\right] \overline{W_{j}\left[\widehat{g}, \widehat{\pi}_{[0, j]}\left(\lambda_{0}\right)\right]}-W_{j}\left[\widehat{f}, \widehat{\pi}_{[0, j]}\left(\lambda_{0}\right)\right] \overline{W_{j}\left[\widehat{g}, \widehat{\xi}_{[0, j]}\left(\lambda_{0}\right)\right]} .
$$

Proof. In the proof, we will omit the indices $[0, j]$ at $\pi$ and $\xi$. At the same time, we will need the following notation used in (3.31):

$$
\xi_{j}(\lambda)=\xi_{j, k_{j}-1}(\lambda), \quad \pi_{j}(\lambda)=\pi_{j, k_{j}-1}(\lambda) .
$$

Formulas (3.32) and (3.66) imply

$$
\begin{aligned}
{\left[f^{\prime}, g\right]-\left[f, g^{\prime}\right]=} & \varepsilon_{j} \widetilde{b}_{j}\left(f_{j+1} \overline{g_{j}}-f_{j} \overline{g_{j+1}}\right) \varepsilon_{j} b_{j}\left(Q_{j+1}\left(\lambda_{0}\right) P_{j}\left(\lambda_{0}\right)-Q_{j}\left(\lambda_{0}\right) P_{j+1}\left(\lambda_{0}\right)\right) \\
= & b_{j}^{2}\left(f_{j+1} \overline{g_{j}}-f_{j} \overline{g_{j+1}}\right)\left(\xi_{j+1}\left(\lambda_{0}\right) \overline{\pi_{j}\left(\lambda_{0}\right)}-\xi_{j}\left(\lambda_{0}\right) \overline{\pi_{j+1}\left(\lambda_{0}\right)}\right) \\
=b_{j}^{2}\left[\left(f_{j+1} \xi_{j}\left(\lambda_{0}\right)-f_{j} \xi_{j+1}\left(\lambda_{0}\right)\right)\left(\overline{g_{j+1} \pi_{j}\left(\lambda_{0}\right)-g_{j} \pi_{j+1}\left(\lambda_{0}\right)}\right)\right. & \\
& \left.\quad-\left(f_{j+1} \pi_{j}\left(\lambda_{0}\right)-f_{j} \pi_{j+1}\left(\lambda_{0}\right)\right)\left(\overline{g_{j+1} \xi_{j}\left(\lambda_{0}\right)-g_{j} \xi_{j+1}\left(\lambda_{0}\right)}\right)\right],
\end{aligned}
$$

thus proving (3.91). 
Proposition 3.27. Each boundary triple for $A_{[0, j]}^{+}$can be represented in the form

$$
\Gamma_{0} \widehat{f}=W_{j}\left[f, \pi\left(\lambda_{0}\right)\right], \quad \Gamma_{1} \widehat{f}=W_{j}\left[f, \xi\left(\lambda_{0}\right)\right], \quad \lambda_{0} \in \mathbb{R} .
$$

The corresponding resolution matrix has the form

$$
\widetilde{\mathcal{W}}_{[0, j]}(\lambda)=\left(\begin{array}{rr}
-W_{j}\left[\xi(\lambda), \pi\left(\lambda_{0}\right)\right] & -W_{j}\left[\xi(\lambda), \xi\left(\lambda_{0}\right)\right] \\
W_{j}\left[\pi(\lambda), \pi\left(\lambda_{0}\right)\right] & W_{j}\left[\pi(\lambda), \xi\left(\lambda_{0}\right)\right]
\end{array}\right)
$$

and is related to the matrix $\mathcal{W}_{[0, j]}(\lambda)$ by the formula

$$
\widetilde{\mathcal{W}}_{[0, j]}(\lambda)=-\mathcal{W}_{[0, j]}(\lambda) \mathcal{W}_{[0, j]}\left(\lambda_{0}\right)^{-1}
$$

Now let us consider the symmetric operator $A:=A_{\min }$ and assume that we have an indefinite case, i.e., $\pi(\lambda) \in \ell^{2}$ for all $\lambda \in \mathbb{C}$. Standard arguments show (see, e.g., 2, 45]) that in this case $\xi(\lambda) \in \ell^{2}$ for all $\lambda \in \mathbb{C}$. The generalized Christoffel-Darboux formulas (3.61)-(3.64) imply the following result.

Proposition 3.28. Let a symmetric operator $A:=A_{\min }$ have defect indices $(1,1)$ and let $\lambda_{0} \in \mathbb{R}$. Then

(1) For each $f \in \mathfrak{H}_{[0, \infty)}$ the limits

$$
W_{\infty}\left[f, \pi\left(\lambda_{0}\right)\right]=\lim _{j \rightarrow \infty} W_{j}\left[f, \pi\left(\lambda_{0}\right)\right], \quad W_{\infty}\left[f, \xi\left(\lambda_{0}\right)\right]=\lim _{j \rightarrow \infty} W_{j}\left[f, \xi\left(\lambda_{0}\right)\right]
$$

exist.

(2) A boundary triple for $A^{+}$can be represented in the form

$$
\Gamma_{0} \widehat{f}=W_{\infty}\left[f, \pi\left(\lambda_{0}\right)\right], \quad \Gamma_{1} \widehat{f}=W_{\infty}\left[f, \xi\left(\lambda_{0}\right)\right] .
$$

(3) The corresponding resolution matrix has the form

$$
\widetilde{\mathcal{W}}_{[0, \infty)}(\lambda)=\left(\begin{array}{rr}
-W_{\infty}\left[\xi(\lambda), \pi\left(\lambda_{0}\right)\right] & -W_{\infty}\left[\xi(\lambda), \xi\left(\lambda_{0}\right)\right] \\
W_{\infty}\left[\pi(\lambda), \pi\left(\lambda_{0}\right)\right] & W_{\infty}\left[\pi(\lambda), \xi\left(\lambda_{0}\right)\right]
\end{array}\right)
$$

(4) The Weyl function of the operator A corresponding to the boundary triple (3.95) has the form

$$
M_{\infty}(\lambda)=-\frac{W_{\infty}\left[\pi(\lambda), \pi\left(\lambda_{0}\right)\right]}{W_{\infty}\left[\pi(\lambda), \xi\left(\lambda_{0}\right)\right]}
$$

Proof. 1. Indeed, (3.32) implies the existence of the limit

$$
\begin{aligned}
W_{\infty}\left[f, \pi\left(\lambda_{0}\right)\right] & =\left[A^{+} f, \pi\left(\lambda_{0}\right)\right]-\lambda_{0}\left[f, \pi\left(\lambda_{0}\right)\right]=\left[\left(A^{+}-\lambda_{0}\right) f, \pi\left(\lambda_{0}\right)\right], \\
W_{\infty}\left[f, \xi\left(\lambda_{0}\right)\right] & =\left[\left(A^{+}-\lambda_{0}\right) f, \xi\left(\lambda_{0}\right)\right]-[f, e] .
\end{aligned}
$$

2. Passing to the limit $j \rightarrow \infty$ in (3.91) we obtain the following formulas for $\widehat{f}=$ $\left\{f, f^{\prime}\right\}, \widehat{g}=\left\{g, g^{\prime}\right\} \in A^{+}:$

$$
\left[f^{\prime}, g\right]-\left[f, g^{\prime}\right]=W_{\infty}[\widehat{f}, \widehat{\xi}] \overline{W_{\infty}[\widehat{g}, \widehat{\pi}]}-W_{\infty}[\widehat{f}, \widehat{\pi}] \overline{W_{\infty}[\widehat{g}, \widehat{\xi}]}
$$

thus proving the second statement of the proposition.

3. Formulas (3.96) and (3.97) follow from (3.54), (3.29), (3.51), and (3.95).

Let $N$ be sufficiently large, so that $H_{[N, \infty)}$ is a classical Jacobi matrix, and let $\widetilde{\mathcal{W}}_{[N, \infty)}$ be the resolution matrix of the operator $A_{[N, \infty)}$ constructed similarly to Proposition 3.28 . The theorem on the factorization of the resolution matrix $\mathcal{W}_{[0, j]}(\lambda)$ and formula (3.94) imply that

$$
\widetilde{\mathcal{W}}_{[0, j]}(\lambda)=\mathcal{W}_{[0, N-1]}(\lambda) \widetilde{\mathcal{W}}_{[N, j]}(\lambda) \mathcal{W}_{[0, N-1]}\left(\lambda_{0}\right)^{-1}
$$


for $N \leq j$. Passing to the limit as $j \rightarrow \infty$ we obtain

$$
\widetilde{\mathcal{W}}_{[0, \infty)}(\lambda)=\mathcal{W}_{[0, N-1]}(\lambda) \widetilde{\mathcal{W}}_{[N, \infty)}(\lambda) \mathcal{W}_{[0, N-1]}\left(\lambda_{0}\right)^{-1} .
$$

Since $\widetilde{\mathcal{W}}_{[N, \infty)}(\lambda)$ is a Nevanlinna matrix of the classical Jacobi matrix $H_{[N, \infty)}$ (see [2]), the known properties of Nevanlinna matrices imply the following result.

Corollary 3.29. The generalized Nevanlinna matrix $\widetilde{\mathcal{W}}_{[0, \infty)}(\lambda)$ is an entire function of minimal exponential type.

Corollary 3.30. Let a symmetric operator $A=A_{\min }$ associated with a Jacobi matrix $H$ have defect indices $(1,1)$. The set of points of regular type of the operator $A$ coincides with $\mathbb{C}$. The operator $A^{(K)}$ with the domain

$$
\operatorname{dom} A^{(K)}=\operatorname{dom} A \dot{+} \operatorname{span}\{\pi(0)\}
$$

given by the formula

$$
A^{(K)}(f+c \pi(0))=A f \quad \text { with } \quad f \in \operatorname{dom} A, \quad c \in \mathbb{C},
$$

is the unique canonical selfadjoint extension of the operator $A$ for which $0 \in \sigma_{p}\left(A^{(K)}\right)$.

Proof. Let the matrix $\widetilde{\mathcal{W}}_{[0, \infty)}(\lambda)$ have the following representation:

$$
\widetilde{\mathcal{W}}_{[0, \infty)}(\lambda)=\left(\widetilde{w}_{i j}(\lambda)\right)_{i, j=1}^{2} .
$$

The functions

$$
M_{\infty}(\lambda)=-\widetilde{w}_{21}(\lambda) / \widetilde{w}_{22}(\lambda) \quad-1 / M_{\infty}(\lambda)=\widetilde{w}_{22}(\lambda) / \widetilde{w}_{21}(\lambda)
$$

are the Weyl functions of the operator $A$. Since

$$
\operatorname{det} \widetilde{\mathcal{W}}_{[0, \infty)}(\lambda)=\widetilde{w}_{11}(\lambda) \widetilde{w}_{22}(\lambda)-\widetilde{w}_{12}(\lambda) \widetilde{w}_{21}(\lambda)=1,
$$

and $\widetilde{w}_{12}(\lambda), \widetilde{w}_{22}(\lambda)$ are entire functions, the functions $\widetilde{w}_{12}(\lambda)$ and $\widetilde{w}_{22}(\lambda)$ have no common zeros and each point in $\mathbb{C}$ is a regular point for at least one of the functions $M_{\infty}(\lambda)$, $-1 / M_{\infty}(\lambda)$. Since $A$ is a simple operator, Theorem 3.7 shows that each point in $\mathbb{C}$ is a regular point either for the selfadjoint extension $A_{0}=\operatorname{ker} \Gamma_{0}$ or for the extension $A_{1}=\operatorname{ker} \Gamma_{1}$. Therefore, $\widehat{\rho}(A)=\mathbb{C}$.

Next, using (3.24) we obtain

$$
\left[A^{+} \xi(0), \pi(0)\right]-\left[\xi(0), A^{+} \pi(0)\right]=\left[e_{0,0}, \pi(0)\right]=1 .
$$

Formula (3.101) shows that the vector $\pi(0)$ does not lie in the domain of the operator $A$ since if we would have had $\pi(0) \in \operatorname{dom} A$, then

$$
[A \xi(0), y]=\left[\xi(0), A^{+} y\right], \quad y \in \operatorname{dom} A^{+} .
$$

In particular, $\pi(0) \in \operatorname{dom} A^{+}$and (3.102) contradicts (3.101).

Therefore the extension $A^{(K)}$ defined by (3.100) is a one-dimensional symmetric extension, and since the defect indices of $A$ are $(1,1)$, a selfadjoint extension of the operator $A$. Moreover, $0 \in \sigma_{p}\left(A^{(K)}\right)$ and the vector $\pi(0)$ is the corresponding eigenvector. Admitting the nonuniqueness of a canonical selfadjoint extension $\widetilde{A}$ with the property $0 \in \sigma_{p}(\widetilde{A})$, we obtain $\pi(0) \in \operatorname{dom} A$, which is impossible since the operator $A$ is simple.

It is known (see [34) that formula (3.53) gives a one-to-one correspondence between the set of all solutions of the complete indefinite moment problem $M_{\kappa}(\mathbf{s})$ and the set of all $u$-resolutions of a symmetric operator $A$. Using the description (3.48) of $u$-resolutions of the operator $A$, we see that in the indefinite case the set of solutions $F$ of the indefinite 
moment problem $M_{\kappa}(\mathbf{s})$ is parameterized by the set of functions $\tau$ of the class $\mathbf{N} \cup\{\infty\}$ according to the formula

$$
F(\lambda)=T_{\widetilde{\mathcal{W}}_{[0, \infty)}}[\tau(\lambda)]
$$

where the fractional linear transformation $T_{\mathcal{W}}[\tau]$ is defined in (3.53).

Using the factorization formula (3.99) we obtain the following relation between the set of all solutions of the moment problem $M_{\kappa}(\mathbf{s})$ and the set of solutions of a certain classical moment problem.

Proposition 3.31. Let the moment problem $M_{\kappa}(\mathbf{s})$ be indefinite and $N>0$ be such that the matrix $S_{n_{N}-1}=\left(s_{i+j}\right)_{i, j=0}^{n_{N}-1}$ has $\kappa$ negative eigenvalues and $H_{[N, \infty)}$ is a classical Jacobi matrix. Then the formula

$$
F(\lambda)=T_{\mathcal{W}_{[0, N-1]}}[\varphi(\lambda)]=\frac{w_{11}(\lambda) \varphi(\lambda)+w_{12}(\lambda)}{w_{21}(\lambda) \varphi(\lambda)+w_{22}(\lambda)}
$$

where $\mathcal{W}_{[0, N-1]}=\left(w_{i j}\right)_{i, j=1}^{2}$ is the resolution matrix of the operator $A_{[0, N-1]}$ defined by (3.60), establishes a one-to-one correspondence between the set $F$ of solutions of the complete indefinite moment problem $M_{\kappa}(\mathbf{s})$ and the set of solutions $\varphi$ of the classical moment problem corresponding to the Jacobi matrix $H_{[N, \infty)}$.

Proof. Indeed, let $F(\lambda)$ be a solution of the indefinite moment problem $M_{\kappa}(\mathbf{s})$, and let it be represented in the form (3.103). Then (3.99) implies that

$$
F(\lambda)=T_{\mathcal{W}_{[0, N-1]}(\lambda)} \circ T_{\widetilde{\mathcal{W}}_{[N, \infty)}(\lambda)} \circ T_{\mathcal{W}_{[0, N-1]}^{-1}\left(\lambda_{0}\right)}[\tau(\lambda)] .
$$

This yields the representation (3.104) if we set

$$
\varphi(\lambda)=T_{\widetilde{\mathcal{W}}_{[N, \infty)}}[\widetilde{\tau}(\lambda)], \quad \widetilde{\tau}(\lambda)=T_{\widetilde{\mathcal{W}}_{[0, N-1]}^{-1}\left(\lambda_{0}\right)}[\tau(\lambda)] .
$$

Since $T_{\widetilde{\mathcal{W}}_{[0, N-1]}^{-1}\left(\lambda_{0}\right)}$ establishes a bijection of the class $\mathbf{N} \cup\{\infty\}$ with itself, we see that formula (3.105) yields a description of the solutions of the classical moment problem corresponding to the Jacobi matrix $H_{[N, \infty)}$.

\section{The Padé approximation}

4.1. Resolution convergence of truncated Jacobi matrices. Let us consider the following finite generalized Jacobi matrix:

$$
H_{[0, j]}(\tau)=\left(\begin{array}{cccc}
A_{0} & \widetilde{B}_{0} & & \mathbf{0} \\
B_{0} & \ddots & \ddots & \\
& \ddots & A_{j-1} & \widetilde{B}_{j-1} \\
\mathbf{0} & & B_{j-1} & A_{j}(\tau)
\end{array}\right), A_{j}(\tau)=\left(\begin{array}{cccc}
0 & \ldots & 0 & -p_{0}^{(j)}+\tau \\
1 & & \mathbf{0} & -p_{1}^{(j)} \\
& \ddots & & \vdots \\
\mathbf{0} & & 1 & -p_{k_{j}-1}^{(j)}
\end{array}\right)
$$

and try to choose the parameter $\tau \in \mathbb{R}$ in such a way that 0 is an eigenvalue of the matrix $H_{[0, j]}(\tau)$. This is possible if

$$
\operatorname{det}\left(H_{[0, j-1]}\right) \neq 0,
$$

which can be equivalently expressed in terms of the polynomials of the first kind as follows: $P_{j}(0) \neq 0$.

Proposition 4.1. If $\operatorname{det}\left(H_{[0, j-1]}\right) \neq 0$, then there exists a unique $\tau_{j} \in \mathbb{R}$ such that the matrix $H_{[0, j]}^{(K)}:=H_{[0, j]}\left(\tau_{j}\right)$ has a zero eigenvalue. 
Proof. The left eigenvector $u \in \ell_{\left[0, n_{j}\right]}^{2}$ of the matrix $H_{[0, j]}(\tau)$ corresponding to the zero eigenvalue should satisfy the system (3.11) for $i=0, \ldots, j-1$. Therefore we can assume that $u_{i k}=P_{i k}(0)\left(i=0, \ldots, j ; k=0, \ldots, k_{j}-1\right)$. In the case $i=j$ we have the following relation:

$$
\widetilde{b}_{j-1} u_{j-1}-\left(p_{j}(0)-\tau\right) u_{j}=0 .
$$

Since $u_{j}=P_{j}(0) \neq 0$, (4.2) implies that

$$
\tau_{j}=-\frac{\widetilde{b}_{j-1} P_{j-1}(0)-p_{j}(0) P_{j}(0)}{P_{j}(0)}=b_{j} \frac{P_{j+1}(0)}{P_{j}(0)} .
$$

Remark 4.2. In the case of a positive Jacobi matrix, the selfadjoint extension $A_{[0, j]}^{(K)}$ of the operator $A_{[0, j]}$ for which $0 \in \sigma_{p}\left(A_{[0, j]}^{(K)}\right)$ is called the Krein extension. We will use this name for arbitrary generalized Jacobi matrices. If the boundary triple for $A_{[0, j]}^{+}$is defined by formula (3.37) and $M(\cdot)$ is the corresponding Weyl function, Corollary 3.8 shows that the extension $A_{[0, j]}^{(K)}$ can be characterized by the following boundary condition (see [21]):

$$
\Gamma_{1} \widehat{f}=M(0) \Gamma_{0} \widehat{f}
$$

where

$$
\widehat{f}=\left\{f, H_{[0, j]} f+\widetilde{b}_{j} f_{j+1} e_{j, 0}\right\} \in A_{[0, j]}^{+}, \quad f \in \mathfrak{H}_{[0, j]}, \quad f_{j+1} \in \mathbb{C} .
$$

Taking into account (3.37) and (3.38), we rewrite condition (4.3) in the form

$$
\varepsilon_{j+1} f_{j+1}=\varepsilon_{j} \frac{P_{j+1}(0)}{P_{j}(0)} f_{j, k_{j}-1} .
$$

Therefore,

$$
\widehat{f}=\left\{f, H_{[0, j]} f+b_{j} \frac{P_{j+1}(0)}{P_{j}(0)} f_{j, k_{j}-1}\right\}=\left\{f, H_{[0, j]}\left(\tau_{j}\right) f\right\} .
$$

This gives another proof of the fact that the matrix $H_{[0, j]}\left(\tau_{j}\right)$ yields a selfadjoint operator $A_{[0, j]}^{(K)}$ in $\mathfrak{H}_{[0, j]}$ such that $0 \in \sigma_{p}\left(A_{[0, j]}^{(K)}\right)$.

Proposition 4.3. Let $H$ be a generalized Jacobi matrix. There exists a number $\eta=$ $\eta(H)>0$ such that for each $j \in \mathbb{Z}_{+}$we have

$$
\left\|\left(H_{[0, j]}-\lambda\right)^{-1}\right\| \leq \frac{1}{|\operatorname{Im} \lambda|-\eta}, \quad|\operatorname{Im} \lambda|>\eta,
$$

and for each $j \in J_{H}=\left\{j \in \mathbb{Z}: \operatorname{det}\left(H_{[0, j-1]}\right) \neq 0\right\}$ we have

$$
\left\|\left(A_{[0, j]}^{(K)}-\lambda\right)^{-1}\right\| \leq \frac{1}{|\operatorname{Im} \lambda|-\eta}, \quad|\operatorname{Im} \lambda|>\eta .
$$

Proof. For each $j \geq N$ such that the operators $H_{[0, j]}$ and $A_{[0, j]}^{(K)}$ exist, they differ from the selfadjoint operators $0 \oplus H_{[N, j]}$ and $0 \oplus H_{[N, j]}\left(\tau_{j}\right)$ in a Hilbert space by the same finite-dimensional operator with the norm not exceeding $\left\|H_{[0, N-1]}\right\|+b_{N-1}$. Therefore, using a well-known result of perturbation theory (see, e.g., [16, p. 76]) we get estimates (4.4) and (4.5) for all $j>N$ with $\eta=\left\|H_{[0, N-1]}\right\|+b_{N-1}$. Using estimates for the norms of resolutions of the operators $H_{[0, j]}$ and $A_{[0, j]}^{(K)}$ with $j \leq N$, we find $\eta=\eta(H)>0$ such that (4.4) and (4.5) hold for all $j \in \mathbb{Z}_{+}$and $j \in J_{H}$ respectively. 
Proposition 4.4. Let $\phi \in \mathfrak{H}_{[0, \infty)}=\ell_{[0, \infty)}^{2}(G)$ and $\lambda \in \Lambda_{\eta}=\{\lambda \in \mathbb{C}:|\operatorname{Im} \lambda|>\eta\}$. Then

(i) if $A$ is a selfadjoint operator in the Pontryagin space $\mathfrak{H}_{[0, \infty)}$, then

$$
\begin{aligned}
& \left(A_{[0, j]}-\lambda\right)^{-1} \phi \rightarrow(A-\lambda)^{-1} \phi \quad \text { as } \quad j \rightarrow \infty ; \\
& \left(A_{[0, j]}^{(K)}-\lambda\right)^{-1} \phi \rightarrow(A-\lambda)^{-1} \phi \quad \text { as } \quad j \rightarrow \infty, j \in J_{H}
\end{aligned}
$$

(ii) if $A$ is a symmetric operator with defect indices $(1,1)$ in the Pontryagin space $\mathfrak{H}_{[0, \infty)}$, then

$$
\left(A_{[0, j]}^{(K)}-\lambda\right)^{-1} \phi \rightarrow\left(A^{(K)}-\lambda\right)^{-1} \phi \quad \text { as } \quad j \rightarrow \infty, j \in J_{H},
$$

where $A^{(K)}$ is a unique selfadjoint extension of the operator $A$ acting in the space $\mathfrak{H}_{[0, \infty)}$ such that $0 \in \sigma\left(A^{(K)}\right)$.

Proof. First let $A$ be a selfadjoint operator in $\mathfrak{H}_{[0, \infty)}$. Then $\operatorname{ran}(A-\lambda)=\mathfrak{H}_{[0, \infty)}$ and it suffices to prove (4.6) and (4.7) on finite vectors. Let $\phi=(A-\lambda) \psi$, where $\psi$ is a finite vector. It is clear that $\phi$ is a finite vector as well. Therefore,

$$
(A-\lambda) \psi=\left(H_{[0, j]}-\lambda\right) \psi=\left(A_{[0, j]}^{(K)}-\lambda\right) \psi=\phi
$$

for sufficiently large $j$. Hence,

$$
\lim _{j \rightarrow \infty}\left(H_{[0, j]}-\lambda\right)^{-1} \phi=(A-\lambda)^{-1} \phi, \quad \lim _{j \rightarrow \infty}\left(A_{[0, j]}^{(K)}-\lambda\right)^{-1} \phi=(A-\lambda)^{-1} \phi
$$

for all finite $\phi \in \mathfrak{H}_{[0, \infty)}$.

Let $A$ be a symmetric operator with defect indices $(1,1)$ and $A^{(K)}$ its selfadjoint extension given by (3.100). Then

$$
\mathfrak{H}_{[0, \infty)}=\operatorname{ran}\left(A^{(K)}-\lambda\right)=\operatorname{ran}(A-\lambda) \dot{+}\{\pi(0)\},
$$

because $\lambda \in \rho\left(A^{(K)}\right)$. Since for $\phi \in \operatorname{ran}(A-\lambda)$ the proof of convergence (4.8) is similar to the case of a selfadjoint operator, it remains to prove (4.8) for $\phi=\pi(0)$. Since $A_{[0, j]}^{(K)} \pi_{[0, j]}(0)=0$ for $j \in J_{H}$, we have

$$
\left(A_{[0, j]}^{(K)}-\lambda\right)^{-1} \pi_{[0, j]}(0)=-\lambda^{-1} \pi_{[0, j]}(0) .
$$

From (4.5) and the fact that $\left\|\pi_{[0, j]}(0)-\pi(0)\right\| \rightarrow 0$ we conclude that

$$
\left(A_{[0, j]}^{(K)}-\lambda\right)^{-1} \pi(0) \rightarrow-\lambda^{-1} \pi(0)=\left(A^{(K)}-\lambda\right)^{-1} \pi(0),
$$

for $\lambda \in \Lambda_{\eta}$ as $j \rightarrow \infty, j \in J_{H}$.

4.2. The Markov theorem. Since each solution $\varphi$ of the moment problem $M_{\kappa}(\mathbf{s})$ has asymptotic expansion (1.5), the formal series

$$
F(\lambda)=-\sum_{j=0}^{\infty} \frac{s_{j}}{\lambda^{j+1}}
$$

will be called the Hamburger series associated to the moment problem $M_{\kappa}(\mathbf{s})$ (see [9]).

Definition 4.5 ([43]). The Padé approximation of type $[L / M]$ for a formal series $-\sum_{j=0}^{+\infty} \frac{s_{j}}{\lambda^{j+1}}$ is the ratio

$$
f^{[L / M]}(\lambda)=\frac{A^{[L / M]}\left(\frac{1}{\lambda}\right)}{B^{[L / M]}\left(\frac{1}{\lambda}\right)}
$$


of two polynomials $A^{[L / M]}$ and $B^{[L / M]}$ of formal degrees $L$ and $M$ respectively such that $B^{[L / M]}(0) \neq 0$ and

$$
\sum_{j=0}^{+\infty} \frac{s_{j}}{\lambda^{j+1}}+f^{[L / M]}(\lambda)=O\left(\lambda^{-(L+M+1)}\right) .
$$

In the case $L=M=n$ the Padé approximation of type $[n / n]$ is called the $n$-th diagonal Padé approximation. The statement concerning the convergence of diagonal Padé approximations for Markov functions is contained in the following classical Markov theorem.

Theorem $4.6([43,9])$. Let $d \mu$ be a nonnegative measure on the interval $[a, b]$ and let

$$
\varphi(\lambda)=\int_{a}^{b} \frac{d \mu(t)}{t-\lambda}
$$

Then the diagonal Padé approximation for the function $\varphi$ takes the form

$$
f^{[n / n]}(\lambda)=-\frac{Q_{n}(\lambda)}{P_{n}(\lambda)}
$$

where $P_{n}$ and $Q_{n}$ are polynomials of the first and second kind constructed using the measure $d \mu$. Padé approximations $f^{[L / M]}$ converge to $\varphi$ locally uniformly on $\mathbb{C} \backslash[a, b]$.

In the case where the support of the measure $d \mu$ is contained in the union of two intervals $[a, b]$ and $[c, d]$, the convergence of diagonal Padé approximations in the interval $(b, c)$ can fail (see, e.g., [44, $\S 3])$. Nevertheless, in the case where the support of the measure $d \mu$ outside the interval $[a, b]$ is discrete, the following corollary of the Markov theorem holds.

Corollary 4.7. Let

$$
\varphi(\lambda)=\int_{a}^{b} \frac{d \mu(t)}{t-\lambda}+\sum_{k=1}^{\beta} \frac{c_{k}}{t_{k}-\lambda},
$$

where $c_{k}>0$ and $t_{k} \in \mathbb{R} \backslash[a, b]$. Then the function $\varphi$ admits diagonal Padé approximations that converge to $\varphi$ locally uniformly on $\mathbb{C} \backslash([a, b] \cup \mathcal{P}(\varphi))$.

Proof. It is clear that $\varphi \in \mathbf{N}$. Let $[\widetilde{a}, \widetilde{b}]$ be the minimal interval of the real line containing the set $\widetilde{E}=[a, b] \cup\left\{t_{1}, \ldots, t_{\beta}\right\}$. Then the function $\varphi(\lambda)$ admits a representation

$$
\varphi(\lambda)=\int_{\widetilde{a}}^{\widetilde{b}} \frac{d \widetilde{\mu}(t)}{t-\lambda}
$$

where the measure $d \widetilde{\mu}$ differs from the measure $d \mu$ by a discrete measure corresponding to the second summand in (4.10). Diagonal Padé approximations for $\varphi$ admit representations

$$
-\frac{\widetilde{Q}_{n}(\lambda)}{\widetilde{P}_{n}(\lambda)}=\int_{\widetilde{a}}^{\widetilde{b}} \frac{d \mu_{n}(t)}{t-\lambda} \in \mathbf{N}
$$

where $\widetilde{P}_{n}$ and $\widetilde{Q}_{n}$ are the first and second kind polynomials corresponding to the measure $d \widetilde{\mu}$. As was mentioned in [44, 2.3], the polynomials $\widetilde{P}_{n}$ have at most $\beta$ zeros outside $[a, b]$. Therefore, there exists a subsequence of fractions $-\widetilde{Q}_{n_{i}} / \widetilde{P}_{n_{i}}$ such that their poles outside $(a, b)$ converge to some points $\lambda_{1}, \ldots, \lambda_{\alpha}$. Let $\delta>0$. Then for a sufficiently large $i\left(>i_{0}\right)$ 
all poles of the function $-\widetilde{Q}_{n_{i}} / \widetilde{P}_{n_{i}}$ lie in the set $E_{\delta}$, the $\delta$-neighborhood of the set $E=[a, b] \cup\left\{\lambda_{1}, \ldots, \lambda_{\alpha}\right\}$. For $\lambda \in \mathbb{C} \backslash E_{2 \delta}$ we have the following estimate:

$$
\left|\frac{\widetilde{Q}_{n_{i}}(\lambda)}{\widetilde{P}_{n_{i}}(\lambda)}\right| \leq \int_{a}^{b} \frac{d \mu_{n_{i}}(t)}{|t-\lambda|}+\sum_{k=1}^{\beta} \frac{c_{k}^{\left(n_{i}\right)}}{\left|t_{k}^{\left(n_{i}\right)}-\lambda\right|} \leq \frac{s_{0}}{\delta},
$$

where

$$
s_{0}=\int_{\widetilde{a}}^{\widetilde{b}} d \widetilde{\mu}(t)=\int_{\widetilde{a}}^{\widetilde{b}} d \mu_{n}(t) .
$$

By the Markov theorem the sequence $-\widetilde{Q}_{n_{i}} / \widetilde{P}_{n_{i}}$ converges to $\varphi$ locally uniformly on $\mathbb{C} \backslash[\widetilde{a}, \widetilde{b}]$. Next, according to (4.11) and the Vitoli theorem, the sequence $-\widetilde{Q}_{n_{i}} / \widetilde{P}_{n_{i}}$ converges to $\varphi$ locally uniformly on $\mathbb{C} \backslash E$. Finally, by the uniqueness theorem for analytic functions, we have $\alpha=\beta$ and $\left\{\lambda_{1} \ldots \lambda_{\beta}\right\}=\left\{t_{1} \ldots t_{\beta}\right\}$.

4.3. Convergence of diagonal Padé approximations for functions in the class $\mathbf{N}_{\kappa,-\infty}$. Suppose we are given a Hamburger series $F$, i.e., a certain moment problem $M_{\kappa}(\mathbf{s})$. To this problem one naturally associates a generalized Jacobi matrix $H$. Representation (3.42) for the $m$-function $m_{[0, j-1]}$ implies that it has an asymptotic expansion

$$
m_{[0, j-1]}(\lambda)=-\frac{Q_{j}(\lambda)}{P_{j}(\lambda)}=-\frac{s_{0}}{\lambda}-\frac{s_{1}}{\lambda^{2}}-\cdots-\frac{s_{2 n_{j}-2}}{\lambda^{2 n_{j}-1}}+O\left(\frac{1}{\lambda^{2 n_{j}}}\right) \quad(\lambda \widehat{\rightarrow} \infty) .
$$

Moreover, as was shown in [20], to the asymptotic expansion (4.12) of the function $m_{[0, j-1]}(\lambda)$ one can add $k_{j}$ additional terms so that it acquires the form

$$
m_{[0, j-1]}(\lambda)=-\sum_{i=0}^{2 n_{j}-2+k_{j}} \frac{s_{i}}{\lambda^{i+1}}+O\left(\frac{1}{\lambda^{2 n_{j}+k_{j}}}\right) \quad(\lambda \widehat{\rightarrow} \infty) .
$$

This formula means that the rational function

$$
f^{\left[n_{j} / n_{j}\right]}(\lambda)=m_{[0, j-1]}(\lambda)=\frac{A^{\left[n_{j} / n_{j}\right]}(1 / \lambda)}{B^{\left[n_{j} / n_{j}\right]}(1 / \lambda)}=\frac{-\frac{1}{\lambda^{n_{j}}} Q_{j}(\lambda)}{\frac{1}{\lambda^{n_{j}}} P_{j}(\lambda)}, \quad j=1,2, \ldots,
$$

is the Padé approximation of type $\left[n_{j} / n_{j}\right]$ for the Hamburger series $F$. Moreover, according to the theorem on the structure of the Padé table (see [9, Theorem 1.4.3]), for $L$ and $M$ satisfying the conditions

$$
L \geq n_{j}, \quad M \geq n_{j}, \quad L+M \leq 2 n_{j}+k_{j}-1,
$$

the Padé approximation of type $[L / M]$ exists and coincides with $f^{\left[n_{j} / n_{j}\right]}$, and for $L$ and $M$ satisfying the conditions

$$
L \leq n_{j}+k_{j}-1, \quad M \leq n_{j}+k_{j}-1, \quad L+M \geq 2 n_{j}+k_{j},
$$

the Padé approximation of type $[L / M]$ does not exist (for a more detailed discussion, see [20]).

Theorem $4.8(20)$. Suppose we are given a Hamburger series $F$ associated with the moment problem $M_{\kappa}(\mathbf{s})$. Let $H$ be the corresponding Jacobi matrix, $n_{j}(j \in \mathbb{N})$ the sequence of normal indices defined by (3.6), and $\eta=\eta(H)$ the number defined by Proposition 4.3 . Then

(i) Padé approximations $f^{\left[n_{j} / n_{j}\right]}$ exist and have the form

$$
f^{\left[n_{j} / n_{j}\right]}(\lambda)=-\frac{Q_{j}(\lambda)}{P_{j}(\lambda)}, \quad j \in \mathbb{N} ;
$$

(ii) the sequence of Padé approximations $f^{[n / n]}\left(n>n_{N}\right)$ is precompact in the topology defined by locally uniform convergence on $\Lambda_{\eta}=\{\lambda \in \mathbb{C}:|\operatorname{Im} \lambda|>\eta\}$; 
(iii) if the moment problem $M_{\kappa}(\mathbf{s})$ is definite, then the sequence of Padé approximations $f^{[n / n]}\left(n>n_{N}\right)$ converges locally uniformly on $\Lambda_{\eta}$ to the unique solution $\varphi$ of the moment problem $M_{\kappa}(\mathbf{s})$.

Proof. (i) The first statement was verified earlier; see (4.13).

(ii) By (4.4), we have

$$
\left|f^{\left[n_{j} / n_{j}\right]}(\lambda)\right|=\left|m_{[0, j-1]}(\lambda)\right|=\left|\left\langle\left(H_{[0, j-1]}-\lambda\right)^{-1} e, e\right\rangle\right| \leq \frac{\|e\|^{2}}{|\operatorname{Im} \lambda|-\eta}, \quad j=1,2, \ldots .
$$

According to (4.15) and the Montel theorem the family of functions $f^{\left[n_{j} / n_{j}\right]}$ is precompact in the topology of uniform convergence on $\Lambda_{\eta}$.

(iii) If the moment problem $M_{\kappa}(\mathbf{s})$ is definite, the corresponding Jacobi matrix generates a selfadjoint operator in $\mathfrak{H}_{[0, \infty)}$. In this case we have the estimate (4.15). Proposition 4.4 and the Vitali theorem imply that the sequence $f^{\left[n_{j} / n_{j}\right]}$ converges locally uniformly on $\Lambda_{\eta}$ to the function $\varphi(\lambda)=\left[(A-\lambda)^{-1} e, e\right]$, which is a solution of the definite moment problem $M_{\kappa}(\mathbf{s})$.

The next theorem generalizes Theorem 4.8 in the situation where the moment problem $M_{\kappa}(\mathbf{s})$ is definite.

Theorem 4.9. Suppose we are given a Hamburger series $F$ associated with the moment problem $M_{\kappa}(\mathbf{s}), \varphi$ a solution of this problem, and $\mathcal{P}(\varphi)$ the set of poles of $\varphi$ in $\mathbb{C}$. Then the Padé approximations $f^{\left[n_{j} / n_{j}\right]}$ for $F$ converge to $\varphi$ locally uniformly on $\mathbb{C} \backslash(\mathbb{R} \cup \mathcal{P}(\varphi))$.

Proof. According to Theorem 4.8(ii) and formula (4.6), the sequence $f^{\left[n_{j} / n_{j}\right]}=m_{[0, j-1]}$ converges locally uniformly on $\Lambda_{\eta}$ to the function $\varphi(\lambda)=\left\langle(H-\lambda)^{-1} e, e\right\rangle$. Next, for $j \geq N+1$ formula (3.21) shows that the function $m_{[0, j-1]}$ admits a representation

$$
m_{[0, j-1]}(\lambda)=\frac{w_{11}(\lambda) m_{[N, j-1]}(\lambda)+w_{12}(\lambda)}{w_{21}(\lambda) m_{[N, j-1]}(\lambda)+w_{22}(\lambda)},
$$

where $\mathcal{W}(\lambda)=\left(w_{i, l}(\lambda)\right)_{i, l=1}^{2}=\mathcal{W}_{[0, N-1]}(\lambda)$ and $m_{[N, j-1]}$ is the Weyl function of the classical Jacobi matrix $H_{[N, j-1]}$. According to Proposition 3.31 the function $\varphi$ admits the following representation:

$$
\varphi(\lambda)=\frac{w_{11}(\lambda) m_{[N,+\infty)}(\lambda)+w_{12}(\lambda)}{w_{21}(\lambda) m_{[N,+\infty)}(\lambda)+w_{22}(\lambda)} .
$$

Formulas (4.16) and (4.17) imply that the sequence $m_{[N, j-1]}$ converges to the function $m_{[N, \infty)} \in \mathbf{N}$ locally uniformly on $\Lambda_{\eta}$. Since $H_{[N, j-1]}$ is a classical Jacobi matrix, $m_{[N, j-1]}$ is a function of the Nevanlinna class and $\left|m_{[N, j-1]}(\lambda)\right| \leq \frac{1}{|\operatorname{Im} \lambda|}$. Next, by the Vitoli theorem we have that $m_{[N, j-1]}$ converges to $m_{[N, \infty)}$ locally uniformly on $\mathbb{C}_{+} \cup \mathbb{C}_{-}$. Now the convergence of the sequence $m_{[0, j-1]}(\lambda)$ to $\varphi(\lambda)$ follows from (4.16) and (4.17).

Remark 4.10. If $M_{\kappa}(\mathbf{s})$ is an indefinite moment problem, then each solution $\varphi$ admits a representation

$$
\varphi(\lambda)=\frac{w_{11}(\lambda) \widetilde{\varphi}(\lambda)+w_{12}(\lambda)}{w_{21}(\lambda) \widetilde{\varphi}(\lambda)+w_{22}(\lambda)}
$$

where $\widetilde{\varphi}$ is a solution of the classical indefinite moment problem corresponding to the classical Jacobi matrix $H_{[N, \infty)}$. According to [45, Theorem 5.29], each partial limit of the sequence $m_{[N, j-1]}$ coincides with some canonical solution of the moment problem $M\left(\mathbf{s}^{(N)}\right)$. Formulas (4.16) and (4.18) imply that each partial limit of the sequence of Padé approximations of type $\left[n_{j} / n_{j}\right]$ for $F$ coincides with a canonical solution of the moment problem $M_{\kappa}(\mathbf{s})$. 
In the next theorem we establish the main result of the paper concerning the convergence of diagonal Padé approximations for a certain subclass of the generalized Nevanlinna class.

Theorem 4.11. Let a function $\varphi$ admit a representation

$$
\varphi(\lambda)=r_{1}(\lambda) \int_{\mathbb{R}} \frac{d \mu(t)}{t-\lambda}+r_{2}(\lambda)
$$

where $\mu$ is a nonnegative measure, $r_{1}=q_{1} / \omega_{1}$ a real rational nonnegative function on $\mathbb{R} \backslash \mathcal{P}\left(r_{1}\right)$ such that $\operatorname{deg} q_{1} \leq \operatorname{deg} \omega_{1}$, and $r_{2}$ is a regular real rational function. If the measure $d \mu$ is a solution of the definite classical Hamburger moment problem, then diagonal Padé approximations converge to $\varphi$ locally uniformly on $\mathbb{C} \backslash(\mathbb{R} \cup \mathcal{P}(\varphi))$.

Proof. Since $\mu$ is a solution of the classical Hamburger moment problem, the Nevanlinna function

$$
\tau(\lambda)=\int_{\mathbb{R}} \frac{d \mu(t)}{t-\lambda}
$$

admits an asymptotic expansion (see [2])

$$
\int_{\mathbb{R}} \frac{d \mu(t)}{t-\lambda} \sim-\frac{\widehat{s}_{0}}{\lambda}-\frac{\widehat{s}_{1}}{\lambda^{2}}-\cdots-\frac{\widehat{s}_{2 n_{j}-2}}{\lambda^{2 n_{j}-1}}+\cdots \quad(\lambda \widehat{\rightarrow} \infty),
$$

with $\widehat{s}_{i} \in \mathbb{R}$. By Proposition 2.1, there is $\kappa \in \mathbb{Z}_{+}$such that the function $\varphi$ belongs to the class $\mathbf{N}_{\kappa,-\infty}$ and admits the asymptotic expansion

$$
\varphi \sim-\frac{s_{0}}{\lambda}-\frac{s_{1}}{\lambda^{2}}-\cdots-\frac{s_{2 n_{j}-2}}{\lambda^{2 n_{j}-1}}+\cdots \quad(\lambda \widehat{\rightarrow} \infty)
$$

with some $s_{i} \in \mathbb{R}$. The resulting moment problem $M_{\kappa}(\mathbf{s})$ is definite. Indeed, if $\varphi_{1}$ and $\varphi_{2}$ are two different solutions of the resulting moment problem, then the functions $\widetilde{\varphi}_{1}=r_{1}^{-1}\left(\varphi_{1}-r_{2}\right)$ and $\widetilde{\varphi}_{2}=r_{1}^{-1}\left(\varphi_{2}-r_{2}\right)$ have an asymptotic expansion (4.20), with $\widetilde{\varphi}_{1} \in \mathbf{N}_{\kappa^{\prime}}$ and $\widetilde{\varphi}_{2} \in \mathbf{N}_{\kappa^{\prime \prime}}$ (it may happen that $\kappa^{\prime} \neq \kappa^{\prime \prime}$ ). Since the moment problem (4.20) has a unique solution in the class $\mathbf{N}$, it has a unique solution in the class $\bigcup_{k=0}^{\infty} \mathbf{N}_{k}$ (see [34, Proposition 1.9]). Therefore, $\kappa^{\prime}=\kappa^{\prime \prime}=0$ and $\widetilde{\varphi}_{1}=\widetilde{\varphi}_{2}$; hence $\varphi_{1}=\varphi_{2}$. Now the result follows from Theorem 4.9.

Corollary 4.12. Under the hypotheses of Theorem 4.11, let the support of the measure $\mu$ lie inside the segment $[a, b]$, i.e., the function $\varphi \in \mathbf{N}_{\kappa,-\infty}$ admits the integral representation

$$
\varphi(\lambda)=r_{1}(\lambda) \int_{a}^{b} \frac{d \mu(t)}{t-\lambda}+r_{2}(\lambda) .
$$

Then the diagonal Padé approximations converge to $\varphi$ locally uniformly on $\mathbb{C} \backslash([a, b] \cup$ $\mathcal{P}(\varphi))$.

Proof. By (4.17) and (4.21), the function $m_{[N, \infty)}$ admits a representation

$$
m_{[N, \infty)}(\lambda)=\int_{a}^{b} \frac{d \mu_{N}(t)}{t-\lambda}+\sum_{k=1}^{\beta} \frac{c_{k}}{t_{k}-\lambda},
$$

where $c_{k}>0, t_{k} \in \mathbb{R} \backslash[a, b]$, and $d \mu_{N}$ is a nonnegative measure on $[a, b]$. By Corollary 4.7. the sequence $m_{[N, j-1]}$ converges to $\varphi$ locally uniformly on $\mathbb{C} \backslash\left([a, b] \cup\left\{t_{1}, \ldots, t_{\beta}\right\}\right)$. Comparing (4.16) with (4.17) we obtain the desired result.

Remark 4.13. In the case where $r_{1} \equiv 1$ and $r_{2}$ has no poles on $[a, b]$, this result was obtained by E. A. Rahmanov [4] (see also [14]). 
The positivity condition for the rational function $r_{1}$ in Theorem 4.11 is essential. The next example shows that Corollary 4.12 is, in general, not true if the function $r_{1}$ in (4.21) changes sign.

Example 4.14 (46]). Let $\alpha_{1}, \alpha_{2}, 1\left(0<\alpha_{1}<\alpha_{2}<1\right)$ be rationally independent numbers. Then the function

$$
\int_{-1}^{1} \frac{\left(t-\cos \pi \alpha_{1}\right)\left(t-\cos \pi \alpha_{2}\right)}{(t-\lambda) \sqrt{1-t^{2}}} d t=\frac{1}{\left(\lambda-\cos \pi \alpha_{1}\right)\left(\lambda-\cos \pi \alpha_{2}\right)}\left(\int_{-1}^{1} \frac{d \mu(t)}{t-\lambda}+a \lambda+b\right)
$$

has all $n$-th Padé approximations. Moreover,

$$
\bigcap_{n=1}^{\infty} \overline{\bigcup_{k \geq n} \mathcal{P}\left(f^{[k / k]}\right)}=\mathbb{C} .
$$

Therefore, the sequence $f^{[k / k]}$ does not converge locally uniformly anywhere.

To formulate a result for the rate of convergence of diagonal Padé approximations we introduce the following notation:

$$
\begin{gathered}
\widetilde{a}:=\min \left\{a, t_{1}, \ldots, t_{\beta}\right\}, \quad \widetilde{b}:=\max \left\{b, t_{1}, \ldots, t_{\beta}\right\}, \\
K_{R}:=\{\lambda \in \mathbb{C}:|\lambda-(\widetilde{a}+\widetilde{b}) / 2|=(\widetilde{b}-\widetilde{a}) R / 2\},
\end{gathered}
$$

where the real numbers $a, b, t_{1}, \ldots, t_{\beta}$ are defined by relation (4.22).

Theorem 4.15. Let all the hypotheses of Corollary 4.12 be satisfied. Then there exists $R_{0}>1$ such that for all $R>R_{0}$ we have

$$
\limsup _{j \rightarrow+\infty}\left|\varphi(\lambda)-m_{[0, j-1]}(\lambda)\right|^{\frac{1}{j}} \leq \frac{1}{R^{2}}, \quad \lambda \in K_{R}
$$

Proof. By (4.16), (4.17), and the fact that $\operatorname{det} W_{[0, N-1]}(\lambda) \equiv 1$ we have

$$
\varphi(\lambda)-m_{[0, j-1]}(\lambda)=\frac{m_{[N, \infty)}(\lambda)-m_{[N, j-1]}(\lambda)}{\left(w_{21}(\lambda) m_{[N, \infty)}(\lambda)+w_{22}(\lambda)\right)\left(w_{21}(\lambda) m_{[N, j-1]}(\lambda)+w_{22}(\lambda)\right)},
$$

where

$$
m_{[N, \infty)}(\lambda)=\int_{\widetilde{a}}^{\widetilde{b}} \frac{d \widetilde{\mu}_{N}(t)}{t-\lambda}
$$

and $m_{[N, j-1]}$ is the $[j-N / j-N]$ Padé approximation for $m_{[N, \infty)}$. Since the function $w_{21} m_{[N, \infty)}+w_{22}$ has a finite number of zeros outside of the interval $[a, b](\subset[\widetilde{a}, \widetilde{b}])$, there exists $R_{0}>1$ such that for all $R>R_{0}$ we have

$$
\left|w_{21}(\lambda) m_{[N, \infty)}(\lambda)+w_{22}(\lambda)\right| \geq c_{R}>0, \quad \lambda \in K_{R} .
$$

Since $w_{21}(\lambda) m_{[N, j-1]}(\lambda)+w_{22}(\lambda) \rightarrow w_{21}(\lambda) m_{[N, \infty)}(\lambda)+w_{22}(\lambda)$ as $j \rightarrow \infty, \lambda \in K_{R}$, there exist $\varepsilon_{R}>0$ and $j_{R} \in \mathbb{N}$ such that

$$
\left|w_{21}(\lambda) m_{[N, j-1]}(\lambda)+w_{22}(\lambda)\right| \geq c_{R}-\varepsilon_{R}>0, \quad j>j_{R} .
$$

By (4.24), (4.25), and (4.26) we have that for $R>R_{0}$ and $j>j_{R}$ the estimate

$$
\left|\varphi(\lambda)-m_{[0, j-1]}(\lambda)\right| \leq \frac{\left|m_{[N, \infty)}(\lambda)-m_{[N, j-1]}(\lambda)\right|}{c_{R}\left(c_{R}-\varepsilon_{R}\right)}, \quad \lambda \in K_{R}
$$

holds. Now inequality (4.23) follows from (4.27) and [43, Thereom 6.2]. 
4.4. Convergence of Padé approximations on the diagonal $[M / M-1]$. The next result describes the behavior of the Padé approximations $f^{[M / M-1]}$ for functions $\varphi$ of class $\mathbf{N}_{\kappa,-\infty}$.

Theorem 4.16. For a given Hamburger series $F$ associated with the moment problem $M_{\kappa}(\mathbf{s})$, the Padé approximation of type $\left[n_{j} / n_{j}-1\right]$ exists if and only if $j \in J_{H}=$ $\left\{j \in \mathbb{Z}: P_{j-1}(0) \neq 0\right\}$. Moreover,

(i) If $M_{\kappa}(\mathbf{s})$ is an indefinite moment problem, then the sequence of Padé approximations $f^{\left[n_{j} / n_{j}-1\right]}\left(j \in J_{H}\right)$ converges locally uniformly on $\mathbb{C} \backslash\left(\mathbb{R} \cup \mathcal{P}\left(\varphi^{(K)}\right)\right)$ to the function $\varphi^{(K)}$, where $\varphi^{(K)}$ is the unique canonical solution of the moment problem $M_{\kappa}(\mathbf{s})$ with a pole at zero, i.e., the solution that admits a representation $\varphi^{(K)}(\lambda)=\left[\left(A^{(K)}-\lambda\right)^{-1} e, e\right]$.

(ii) If $M_{\kappa}(\mathbf{s})$ is a definite moment problem, then the sequence of Padé approximations $f^{\left[n_{j} / n_{j}-1\right]}\left(j \in J_{H}\right)$ converges locally uniformly on $\mathbb{C} \backslash(\mathbb{R} \cup \mathcal{P}(\varphi))$ to the unique solution $\varphi$ of the moment problem $M_{\kappa}(\mathbf{s})$.

Proof. Existence. Let $P_{j-1}(0) \neq 0$ and let $P_{j}^{(K)}, Q_{j}^{(K)}$ be polynomials of the first and second kind constructed using the matrix $H_{[0, j-1]}^{(K)}$. Then formula (3.42) implies that

$$
m_{[0, j-1]}^{(K)}(\lambda)=-\frac{Q_{j}^{(K)}(\lambda)}{P_{j}^{(K)}(\lambda)}=\left[\left(H_{[0, j-1]}^{(K)}-\lambda\right)^{-1} e, e\right] \sim-\sum_{i=0}^{\infty} \frac{s_{i}{ }^{(K)}}{\lambda^{i+1}},
$$

where $s_{i}{ }^{(K)}=\left[\left(H_{[0, j-1]}^{(K)}\right)^{i} e, e\right]$. The form of the matrix $H_{[0, j-1]}^{(K)}$ implies that

$$
s_{i}^{(K)}=s_{i} \quad \text { for } \quad i \leq 2 n_{j}-2, \quad s_{2 n_{j}-1}^{(K)}=s_{2 n_{j}-1}+\left(b_{0} \ldots b_{j-1}\right)^{2} \varepsilon_{j-1} \tau_{j-1} .
$$

Therefore, the function $m_{[0, j-1]}^{(K)}(\lambda)$ has the following asymptotic expansion:

$$
m_{[0, j-1]}^{(K)}(\lambda)=-\sum_{i=0}^{2 n_{j}-2} \frac{s_{i}}{\lambda^{i+1}}+O\left(\frac{1}{\lambda^{2 n_{j}}}\right) \quad(\lambda \widehat{\rightarrow} \infty) .
$$

Setting

$$
A^{\left[n_{j} / n_{j}-1\right]}\left(\frac{1}{\lambda}\right)=\left(\frac{1}{\lambda}\right)^{n_{j}} Q_{j}^{(K)}(\lambda), \quad B^{\left[n_{j} / n_{j}-1\right]}\left(\frac{1}{\lambda}\right)=\left(\frac{1}{\lambda}\right)^{n_{j}} P_{j}^{(K)}(\lambda),
$$

and taking into account that $P_{j}^{(K)}(\lambda)=0$, we obtain the equality

$$
m_{[0, j-1]}^{(K)}(\lambda)=\frac{A^{\left[n_{j} / n_{j}-1\right]}(1 / \lambda)}{B^{\left[n_{j} / n_{j}-1\right]}(1 / \lambda)},
$$

where

$$
\operatorname{deg} A^{\left[n_{j} / n_{j}-1\right]}=n_{j}, \quad \operatorname{deg} B^{\left[n_{j} / n_{j}-1\right]}=n_{j}-1, \quad B^{\left[n_{j} / n_{j}-1\right]}(0)=\frac{1}{b_{0} \ldots b_{j-1}} \neq 0 .
$$

Therefore, the function $m_{[0, j-1]}^{(K)}(\lambda)$ is the Padé approximation of type $\left[n_{j} / n_{j}-1\right]$.

Now let us assume that the Padé approximation $f^{\left[n_{j} / n_{j}-1\right]}$ exists. Then

$$
f^{\left[n_{j} / n_{j}-1\right]}(\lambda)=\frac{A^{\left[n_{j} / n_{j}-1\right]}\left(\frac{1}{\lambda}\right)}{B^{\left[n_{j} / n_{j}-1\right]}\left(\frac{1}{\lambda}\right)}=\frac{C(\lambda)}{D(\lambda)}=-\sum_{i=0}^{2 n_{j}-2} \frac{s_{i}}{\lambda^{i+1}}+O\left(\frac{1}{\lambda^{2 n_{j}}}\right) \quad(\lambda \widehat{\rightarrow} \infty),
$$

where $C$ and $D$ are polynomials of degree $n_{j}-1$ and $n_{j}$ respectively, and $D(0)=0$. By Theorem 2.3, the rational function $f^{\left[n_{j} / n_{j}-1\right]}$ admits the following representation as a 
finite $P$-fraction:

$$
f^{\left[n_{j} / n_{j}-1\right]}(\lambda)=-\frac{\varepsilon_{0}}{p_{0}(\lambda)}-\frac{\varepsilon_{0} \varepsilon_{1} b_{0}^{2}}{p_{1}(\lambda)}-\ldots \quad-\frac{\varepsilon_{j-3} \varepsilon_{j-2} b_{j-3}^{2}}{p_{j-2}(\lambda)}-\frac{\varepsilon_{j-2} \varepsilon_{j-1} b_{j-2}^{2}}{\widetilde{p}_{j-1}(\lambda)} .
$$

Using this expansion, we can reconstruct the finite generalized Jacobi matrix $\widetilde{H}_{[0, j-1]}$; the matrix $\widetilde{H}_{[0, j-1]}$ is such that $\widetilde{H}_{[0, j-2]}=H_{[0, j-2]}$, and 0 is an eigenvalue of $\widetilde{H}_{[0, j-1]}$ because $D(0)=0$. Therefore, for the polynomials of the first kind $P_{j}^{\widetilde{H}}$ constructed from $\widetilde{H}_{[0, j-1]}$ we have $P_{j}^{\widetilde{H}}(0)=0$. By Proposition 3.4 we have $P_{j-1}^{\widetilde{H}}(0) \neq 0$. Since $\widetilde{H}_{[0, j-2]}=H_{[0, j-2]}$, we also have $P_{j-1}(0)=P_{j-1}^{\widetilde{H}}(0) \neq 0$.

According to Proposition 3.4. there exist infinitely many $j$ such that $P_{j-1}(0) \neq 0$; indeed if $P_{j-1}(0)=0$, then $P_{j}(0) \neq 0$. Therefore, it makes sense to talk about convergence.

Convergence. By (4.5), the family of functions $f^{\left[n_{j} / n_{j}-1\right]}=m_{[0, j-1]}^{(K)}$ is uniformly bounded on each compact set in $\Lambda_{\eta}$. Therefore, by the Montel theorem the sequence $f^{\left[n_{j} / n_{j}-1\right]}$ is a precompact set in the topology of locally uniform convergence in $\Lambda_{\eta}$. Moreover, by Proposition 4.4 the sequence $f^{\left[n_{j} / n_{j}-1\right]}$ converges locally uniformly in $\Lambda_{\eta}$. Corollary 3.21 shows that for $j \geq N+1$, the function $m_{[0, j-1]}^{(K)}$ admits a representation

$$
m_{[0, j-1]}^{(K)}(\lambda)=\frac{w_{11}(\lambda) m_{[N, j-1]}^{\left(\tau_{j-1}\right)}(\lambda)+w_{12}(\lambda)}{w_{21}(\lambda) m_{[N, j-1]}^{\left(\tau_{j-1}\right)}(\lambda)+w_{22}(\lambda)},
$$

where $m_{[N, j-1]}^{\left(\tau_{j-1}\right)}$ is the Weyl function of the classical Jacobi matrix $H_{[N, j-1]}\left(\tau_{j-1}\right)$. Formula (4.28) shows that $m_{[N, j-1]}^{\left(\tau_{j-1}\right)}$ converges locally uniformly in $\Lambda_{\eta}$. Since $H_{[N, j-1]}\left(\tau_{j-1}\right)$ is a classical Jacobi matrix, $m_{[N, j-1]}^{\left(\tau_{j-1}\right)}$ is a function of Nevanlinna class and

$$
\left|m_{[N, j-1]}^{\left(\tau_{j-1}\right)}(\lambda)\right| \leq \frac{1}{|\operatorname{Im} \lambda|} .
$$

Next, by the Vitali theorem, $m_{[N, j-1]}^{\left(\tau_{j-1}\right)}$ converges locally uniformly in $\mathbb{C}_{+} \cup \mathbb{C}_{-}$. Finally, from (4.28) it follows that the sequence $f^{\left[n_{j} / n_{j}-1\right]}$ also converges locally uniformly on $\mathbb{C} \backslash(\mathbb{R} \cup \mathcal{P}(\varphi))$.

Corollary 4.17. Let a function $\varphi$ admit the integral representation (4.19) with a measure $d \mu$ that is a solution of a definite classical Hamburger moment problem. Then the Padé approximation $f^{[n / n-1]}$ converges to $\varphi$ locally uniformly on $\mathbb{C} \backslash(\mathbb{R} \cup \mathcal{P}(\varphi))$.

The next example shows that for the Padé approximations $f^{\left[n_{j} / n_{j}-1\right]}$ we do not have an analog of Corollary 4.12,

Example 4.18. Consider the following classical 2-periodic Jacobi matrix:

$$
H=\left(\begin{array}{ccccc}
a_{0} & b_{0} & & & \mathbf{0} \\
b_{0} & a_{1} & b_{1} & & \\
& b_{1} & a_{2} & \ddots & \\
\mathbf{0} & & \ddots & \ddots &
\end{array}\right), \quad a_{n}=(-1)^{n}, \quad b_{n}=1, \quad n \in \mathbb{Z}_{+} .
$$

The $m$-function corresponding to this Jacobi matrix can be found using standard methods (see [43):

$$
\varphi(\lambda)=\left((H-\lambda)^{-1} e, e\right)_{\ell^{2}}=\frac{\lambda-\lambda^{2}+\sqrt{\left(\lambda^{2}-\lambda-2\right)^{2}-4}}{2(\lambda-1)}
$$


(here the branch of the square root is determined by the condition $\varphi(\lambda) \sim-1 / \lambda$ as $|\lambda| \rightarrow+\infty)$. Therefore, the function $\varphi(\lambda)$ admits an integral representation (1.15) with a measure $d \sigma$ that has a compact support lying in $[-2,3]$. Since in this case the Padé approximation of type $[n / n-1]$ is of the form $f^{[n / n-1]}(\lambda)=m_{[0, n-1]}^{(K)}(\lambda)$, its poles coincide with eigenvalues of the matrix $H_{[0, n-1]}^{(K)}$. Let us show that the largest absolute values of eigenvalues of the matrix $H_{[0,2 k]}^{(K)}$ tend to infinity as $k \rightarrow+\infty$. First we compute $\tau_{n}$ (see 4.1). Since $\tau_{n}=b_{n} P_{n+1}(0) / P_{n}(0)=-b_{n-1} \widetilde{b}_{n-1} / \tau_{n-1}+p_{n}(0)$, in our case we have

$$
\tau_{n}=-\frac{1}{\tau_{n-1}}+\frac{(-1)^{n}+1}{2} .
$$

It is clear that $\tau_{0}=-1$. By induction, we have the following formulas:

$$
\tau_{2 k}=-(k+1), \quad \tau_{2 k+1}=\frac{1}{k+1} .
$$

Taking into account that $H_{[0,2 k]}^{(K)}$ is a selfadjoint matrix,

$$
\left|\lambda_{\max }\left(H_{[0,2 k]}^{(K)}\right)\right|=\left\|H_{[0,2 k]}^{(K)}\right\| \geq\left|\left(H_{[0,2 k]}^{(K)} e_{2 k}, e_{2 k}\right)\right|=k,
$$

where $\lambda_{\max }\left(H_{[0,2 k]}^{(K)}\right)$ is the eigenvalue of the matrix $H_{[0,2 k]}^{(K)}$ with the largest absolute value. Therefore, $\left|\lambda_{\max }\left(H_{[0,2 k]}^{(K)}\right)\right| \rightarrow+\infty$ as $k \rightarrow+\infty$ and infinity is an accumulation point of the set of poles of the Padé approximations $f^{[n / n-1]}$ of the function $\varphi$ holomorphic at infinity.

4.5. Convergence of paradiagonal Padé approximation. For an arbitrary Laurent series

$$
\Phi(\lambda)=\sum_{j=-\infty}^{+\infty} \frac{c_{j}}{\lambda^{j+1}},
$$

define the projection $\mathbb{P}_{i ; k}$ sending a series $\Phi$ to the partial sum

$$
\left(\mathbb{P}_{i ; k} \Phi\right)(\lambda)=\sum_{j=i}^{k} \frac{c_{j}}{\lambda^{j+1}} .
$$

Let $k \in \mathbb{Z}$. Then the Padé approximation $f^{[L-k / M]}$ of type $[L-k / M]$ for the formal series $F$ can be expressed in terms of the Padé approximation $f_{k}^{[L / M]}$ of type $[L / M]$ for the series

$$
F_{k}(\lambda):=\mathbb{P}_{0 ;+\infty}\left(\lambda^{-k} F(\lambda)\right) .
$$

Namely, we have the following result (see 41]).

Lemma 4.19. Suppose we are given a formal series $F(\lambda)=-\sum_{j=0}^{+\infty} \frac{s_{j}}{\lambda^{j+1}}$ and let $f_{k}^{[L / M]}$ denote the Padé approximation of type $[L / M]$ for the series (4.29). Then for each $k \in \mathbb{Z}$ we have

$$
f^{[L-k / M]}=\lambda^{k} f_{k}^{[L / M]}+\lambda^{k} \mathbb{P}_{-\infty ;-1}\left(\lambda^{-k} F(\lambda)\right),
$$

provided $L \geq M$ and one of the Padé approximations $f^{[L-k / M]}$ or $f_{k}^{[L / M]}$ exists.

Proof. First let us assume that for the series $F_{k}$ there exists the Padé approximation $f_{k}^{[L / M]}$, i.e., there exist polynomials $A^{[L / M]}$ and $B^{[L / M]}$ of formal degrees $L$ and $M$ respectively such that $B^{[L / M]}(0) \neq 0$ and

$$
F_{k}(\lambda)-\frac{A^{[L / M]}(1 / \lambda)}{B^{L / M]}(1 / \lambda)}=O\left(\frac{1}{\lambda^{L+M+1}}\right) .
$$


Let $k_{0} \in \mathbb{N}$ be chosen in such a way that $s_{0}=\cdots=s_{k_{0}-2}=0$ and $s_{k_{0}-1} \neq 0$. First let us assume that $-k_{0}<k \leq 0$. Then (4.31) can be rewritten in the following form:

$$
\lambda^{-k} F(\lambda)-\frac{A^{[L / M]}(1 / \lambda)}{B^{[L / M]}(1 / \lambda)}=O\left(\frac{1}{\lambda^{L+M+1}}\right) .
$$

Multiplying both sides of this formula by $\lambda^{k}$, we obtain

$$
F(\lambda)-\frac{\widetilde{A}\left(\frac{1}{\lambda}\right)}{B^{[L / M]}\left(\frac{1}{\lambda}\right)}=O\left(\frac{1}{\lambda^{L+M-k+1}}\right),
$$

where $\widetilde{A}(1 / \lambda):=(1 / \lambda)^{-k} A^{[L / M]}(1 / \lambda)$ is a polynomial of formal degree $L-k$ with respect to $1 / \lambda$, and $B^{[L / M]}(0) \neq 0$. Therefore, by (4.33) the series $F$ admits the Padé approximation $f^{[L-k / M]}$ and

$$
f^{[L-k / M]}(\lambda)=\lambda^{k} f_{k}^{[L / M]}(\lambda) .
$$

Now for $k \leq-k_{0}$, let $r_{k}(\lambda)=\mathbb{P}_{-\infty ;-1}\left(\lambda^{-k} F(\lambda)\right)$ be a polynomial of degree $-\left(k+k_{0}\right)$ with respect to $\lambda$. Then $F_{k}(\lambda)=\lambda^{-k} F(\lambda)-r_{k}(\lambda)$. Substituting this equality in (4.31) and multiplying by $\lambda^{k}$ we obtain

$$
F(\lambda)-\frac{\lambda^{k} r_{k}(\lambda) B^{[L / M]}(1 / \lambda)+\lambda^{k} A^{[L / M]}(1 / \lambda)}{B^{[L / M]}(1 / \lambda)}=O\left(\frac{1}{\lambda^{L+M-k+1}}\right) .
$$

The degree of the polynomial $C(1 / \lambda):=\lambda^{k} r_{k}(\lambda) B^{[L / M]}(1 / \lambda)+\lambda^{k} A^{[L / M]}(1 / \lambda)$ does not exceed $L-k$ since $L \geq M$. Therefore, the rational function

$$
f^{[L-k / M]}(\lambda)=\frac{C(1 / \lambda)}{B^{[L / M]}(1 / \lambda)}
$$

is a Padé approximation of type $[L-k / M]$ for $F$ because $\operatorname{deg} C \leq L-k, \operatorname{deg} B^{[L / M]} \leq M$. Moreover,

$$
f^{[L-k / M]}(\lambda)=\lambda^{k} r_{k}(\lambda)+\lambda^{k} f_{k}^{[L / M]}(\lambda) .
$$

Finally, let $k>0$. Then formula (4.31) is equivalent to (4.32). By (4.32),

$$
A^{[L / M]}\left(\frac{1}{\lambda}\right)=O\left(\frac{1}{\lambda^{k}}\right),
$$

so that $\widetilde{A}(\lambda):=\lambda^{-k} A^{[L / M]}(\lambda)$ is a polynomial of formal degree $L-k$. Therefore, (4.32) implies (4.33) with $\operatorname{deg} B^{[L / M]} \leq M, B^{[L / M]}(0) \neq 0$. Therefore the Padé approximation of type $[L-k / M]$ for the series $F$ exists and can be found by formula (4.30).

The case $[L-k / M]$ is treated similarly.

To pass to an arbitrary diagonal of the Padé table for the Hamburger series $F$ consider the following formal series:

$$
F_{2 l}(\lambda)=\mathbb{P}_{0 ;+\infty}\left(\lambda^{-2 l} F(\lambda)\right)=-\sum_{j=0}^{+\infty} \frac{s_{j}(l)}{\lambda^{j+1}},
$$

corresponding to the sequence of moments $\mathbf{s}(l)$ defined by the formulas

(i) if $l \in \mathbb{N}$, then $s_{0}(l)=\cdots=s_{2 l-1}(l)=0, s_{2 l+i}(l)=s_{i}, i \in \mathbb{Z}_{+}$;

(ii) if $l=0$, then $s_{i}(0)=s_{i}, i \in \mathbb{Z}_{+}$, i.e., $\mathbf{s}(0)=\mathbf{s}$;

(iii) if $-l \in \mathbb{N}$, then $s_{0}(l)=s_{-2 l}, s_{1}(l)=s_{1-2 l}, \ldots, s_{i}(l)=s_{i-2 l}, i \in \mathbb{Z}_{+}$. 
Define $\kappa(l)$ as the maximal number of negative eigenvalues of the matrix

$$
\left(s_{i+j}(l)\right)_{i, j=0}^{n}, \quad n=0,1,2, \ldots .
$$

The definition of the sequence $\mathbf{s}(l)$ implies that $\kappa(l) \leq \kappa:=\kappa(0)$ for $l<0$. For $l>0$ the number $\kappa(l)$ may be larger than $\kappa$, but not more than by $l$.

Proposition 4.20. The moment problem $\mathbf{M}_{\kappa(l)}(\mathbf{s}(l))$ corresponding to the sequence $\mathbf{s}(l)$ is definite if and only if the moment problem $\mathbf{M}_{\kappa}(\mathbf{s})$ is definite.

Proof. If $\varphi$ is a solution of the moment problem $\mathbf{M}_{\kappa}(\mathbf{s})$, then the function

$$
\varphi(\lambda, l)=\lambda^{-2 l} \varphi(\lambda)-r_{l}(\lambda), \quad r_{l}(\lambda):=\mathbb{P}_{-\infty ;-1}\left(\lambda^{-2 l} F(\lambda)\right),
$$

lies in some class $\mathbf{N}_{\kappa(l),-\infty}$, i.e., is a solution of the moment problem $\mathbf{M}_{\kappa(l)}(\mathbf{s}(l))$. Let us assume that the moment problem $\mathbf{M}_{\kappa(l)}(\mathbf{s}(l))$ is indefinite, whereas the problem $\mathbf{M}_{\kappa}(\mathbf{s})$ is definite. Let $\varphi_{1}$ and $\varphi_{2}$ be two different solutions of the moment problem $\mathbf{M}_{\kappa(l)}(\mathbf{s}(l))$. Then the functions $\widetilde{\varphi}_{1}=\lambda^{2 l}\left(\varphi_{1}(\lambda)-r_{l}(\lambda)\right)$ and $\widetilde{\varphi}_{2}=\lambda^{2 l}\left(\varphi_{2}(\lambda)-r_{l}(\lambda)\right)$ are two different functions of classes $\mathbf{N}_{\kappa^{\prime}}$ and $\mathbf{N}_{\kappa^{\prime \prime}}$ (possibly, $\kappa^{\prime} \neq \kappa^{\prime \prime}$ ) and have the same asymptotics (4.36) as $\lambda \widehat{\rightarrow} \infty$. This contradicts Proposition 1.9 in [34], and we obtain the desired result.

Therefore, the series $F_{2 l}(\lambda)=\mathbb{P}_{0 ;+\infty}\left(\lambda^{-2 l} F(\lambda)\right)$ is the Hamburger series associated with the moment problem $\mathbf{M}_{\kappa(l)}(\mathbf{s}(l))$.

By $P_{j}(\lambda, l)$ we denote the polynomials of the first kind of degree $n_{j}(l)$ constructed by the sequence $\mathbf{s}(l)$. The degrees $n_{j}(l)$ form the sequence of normal indices for the matrix $\left(s_{i+j}(l)\right)_{i, j=0}^{\infty}$.

Theorem 4.21. Suppose we are given a Hamburger series $F$ associated with the moment problem $\mathbf{M}_{\kappa}(\mathbf{s}), l \in \mathbb{Z}$. Then

(i) There exist Padé approximations of type $\left[n_{j}(l)-2 l / n_{j}(l)\right]$. Moreover, there exists $\eta(l)>0$ such that the sequence $f^{\left[n_{j}(l)-2 l / n_{j}(l)\right]}$ is precompact in the topology of local uniform convergence on $\Lambda_{\eta(l)}=\{\lambda \in \mathbb{C}:|\operatorname{Im} \lambda|>\eta(l)\}$.

(ii) If the moment problem $M_{\kappa}(\mathbf{s})$ is definite, then the sequence $f^{\left[n_{j}(l)-2 l / n_{j}(l)\right]}$ converges locally uniformly on $\mathbb{C} \backslash(\mathbb{R} \cup \mathcal{P}(\varphi))$ to the unique solution $\varphi$ of the moment problem $M_{\kappa}(\mathbf{s})$.

Proof. As we have already noticed, $F_{2 l}(\lambda)=\mathbb{P}_{0 ;+\infty}\left(\lambda^{-2 l} F(\lambda)\right)$ is the Hamburger series associated with the moment problem $\mathbf{M}_{\kappa(l)}(\mathbf{s}(l))$. By Theorem 4.8 the series $F_{2 l}$ admits the Padé approximation $f^{\left[n_{j}(l) / n_{j}(l)\right]}$. Lemma 4.19 implies the existence of the Padé approximation $f^{\left[n_{j}(l)-2 l / n_{j}(l)\right]}(\lambda)$ for the Hamburger series $F$,

$$
f^{\left[n_{j}(l)-2 l / n_{j}(l)\right]}(\lambda)=\lambda^{2 l} f_{2 l}^{\left[n_{j}(l) / n_{j}(l)\right]}(\lambda)+\lambda^{2 l} \mathbb{P}_{-\infty ;-1}\left(\lambda^{-2 l} F(\lambda)\right) .
$$

Precompactness of the sequence $f^{\left[n_{j}(l)-2 l / n_{j}(l)\right]}$ follows from formula (4.37) and part (ii) of Theorem 4.8 .

To prove part (ii) of Theorem 4.21 it suffices to note that by Proposition 4.20 the moment problem $\mathbf{M}_{\kappa(l)}(\mathbf{s}(l))$ is definite provided the problem $M_{\kappa}(\mathbf{s})$ is definite. Let $l \geq 0$ and $\varphi(\cdot, l)$ be the unique solution of the moment problem $\mathbf{M}_{\kappa(l)}(\mathbf{s}(l))$ with the asymptotics

$$
\varphi(\lambda, l)=-\frac{s_{0}}{\lambda^{2 l+1}}-\frac{s_{1}}{\lambda^{2 l+2}}-\cdots-\frac{s_{2 n}}{\lambda^{2 l+2 n+1}}+o\left(\frac{1}{\lambda^{2 l+2 n+1}}\right) \quad(\lambda \widehat{\rightarrow} \infty) .
$$

By Theorem 4.9 the sequence $f_{2 l}^{\left[n_{j}(l) / n_{j}(l)\right]}(\lambda)$ converges to the function $\varphi(\lambda, l)$ locally uniformly on $\mathbb{C} \backslash(\mathbb{R} \cup \mathcal{P}(\varphi(\cdot, l)))$. Formula (4.37), which for $l \geq 0$ takes the form

$$
f^{\left[n_{j}(l)-2 l / n_{j}(l)\right]}(\lambda)=\lambda^{2 l} f_{2 l}^{\left[n_{j}(l) / n_{j}(l)\right]}(\lambda),
$$


implies that the sequence $f^{\left[n_{j}(l)-2 l / n_{j}(l)\right]}(\lambda)$ converges to the function

$$
\varphi(\lambda)=\lambda^{2 l} \varphi(\lambda, l),
$$

which, by (4.38), is a solution of the moment problem $\mathbf{M}_{\kappa}(\mathbf{s})$. Let us note that by (4.39), $\mathcal{P}(\varphi(\cdot, l)) \backslash \mathbb{R}=\mathcal{P}(\varphi) \backslash \mathbb{R}$.

Similarly, for $l<0$, Theorem 4.9 shows that the sequence $f_{2 l}^{\left[n_{j}(l) / n_{j}(l)\right]}(\lambda)$ converges to the function $\varphi(\lambda, l)$ with the asymptotics

$$
\varphi(\lambda, l)=-\frac{s_{-2 l}}{\lambda}-\frac{s_{-2 l+1}}{\lambda^{2}}-\cdots-\frac{s_{-2 l+2 n}}{\lambda^{2 n+1}}+o\left(\frac{1}{\lambda^{2 n+1}}\right) \quad(\lambda \widehat{\rightarrow} \infty)
$$

for each sufficiently large $n \in \mathbb{N}$. Therefore, the sequence $f^{\left[n_{j}(l)-2 l / n_{j}(l)\right]}(\lambda)$, which according to (4.37) takes the form

$$
f^{\left[n_{j}(l)-2 l / n_{j}(l)\right]}(\lambda)=-\frac{s_{0}}{\lambda}-\cdots-\frac{s_{-2 l-1}}{\lambda^{-2 l}}+\lambda^{2 l} f_{2 l}^{\left[n_{j}(l) / n_{j}(l)\right]}(\lambda),
$$

converges locally uniformly on $\mathbb{C} \backslash(\mathbb{R} \cup \mathcal{P}(\varphi))$ to the function $\varphi(\lambda)$ with the asymptotics (1.5).

Theorem 4.22. Suppose we are given a Hamburger series $F$ associated with the moment problem $M_{\kappa}(\mathbf{s})$, and let $l \in \mathbb{Z}$. Then the Padé approximation of type $\left[n_{j}(l)-2 l / n_{j}(l)-1\right]$ exists if and only if $j \in J_{H(l)}=\left\{j \in \mathbb{Z}: P_{j-1}(0, l) \neq 0\right\}$. Moreover,

(i) If the problem $M_{\kappa}(\mathbf{s})$ is indefinite, then the sequence of Padé approximations $f^{\left[n_{j}(l)-2 l / n_{j}(l)-1\right]}\left(j \in J_{H(l)}\right)$ converges locally uniformly on $\mathbb{C} \backslash\left(\mathbb{R} \cup \mathcal{P}\left(\varphi_{l}\right)\right)$ to a function $\varphi_{l}$, where

$$
\begin{gathered}
\varphi_{l}(\lambda)=\lambda^{2 l} \varphi^{(K)}(\lambda, l) \quad \text { for } \quad l \geq 0, \\
\varphi_{l}(\lambda)=-\frac{s_{0}}{\lambda}-\cdots-\frac{s_{-2 l-1}}{\lambda^{-2 l}}+\lambda^{2 l} \varphi^{(K)}(\lambda, l) \quad \text { for } \quad l<0,
\end{gathered}
$$

and $\varphi^{(K)}(\lambda, l)$ is a unique canonical solution of the moment problem $\mathbf{M}_{\kappa(l)}(\mathbf{s}(l))$ with pole at zero.

(ii) If the problem $M_{\kappa}(\mathbf{s})$ is definite, then the sequence of Padé approximations $f^{\left[n_{j}(l) / n_{j}(l)-1\right]}\left(j \in J_{H(l)}\right)$ converges locally uniformly on $\mathbb{C} \backslash(\mathbb{R} \cup \mathcal{P}(\varphi))$ to the unique solution $\varphi$ of the moment problem $\mathbf{M}_{\kappa}(\mathbf{s})$.

Proof. By Theorem 4.16, the Padé approximation $\left[n_{j}(l) / n_{j}(l)-1\right]$ of type $F_{2 l}$ for the series $F_{2 l}$ exists if and only if $P_{j-1}(0, l) \neq 0$. If the Padé approximation $\left[n_{j}(l) / n_{j}(l)-1\right]$ exists, then, by Theorem 4.19 we obtain

$$
f^{\left[n_{j}(l)-2 l / n_{j}(l)-1\right]}(\lambda)=\lambda^{2 l} f_{2 l}^{\left[n_{j}(l) / n_{j}(l)-1\right]}(\lambda)+\lambda^{2 l} \mathbb{P}_{-\infty ;-1}\left(\lambda^{-2 l} F(\lambda)\right),
$$

where $f_{2 l}^{\left[n_{j}(l) / n_{j}(l)-1\right]}(\lambda)$ is the Padé approximation of type $\left[n_{j}(l) / n_{j}(l)-1\right]$ for the Hamburger series $F_{2 l}$. Finally, convergence of the sequence $f^{\left[n_{j}(l)-2 l / n_{j}(l)-1\right]}$ follows from formula (4.41) and Theorem 4.16.

Corollary 4.23. Let a function $\varphi$ admit an integral representation (4.19), where the measure $d \mu$ is a solution of the definite classical Hamburger moment problem $l \in \mathbb{Z}$. Then the Padé approximation $f^{[n-l / n]}$ converges to $\varphi$ locally uniformly on $\mathbb{C} \backslash(\mathbb{R} \cup \mathcal{P}(\varphi))$.

\section{Appendix A. Factorization of the Resolution matrix}

For the first time a relation between the $u$-resolution of a matrix and the characteristic function of a certain $\mathfrak{J}$-colligation was found in [37; see also [25]. In this appendix we will use the definition of the characteristic function of a linear relation in a Pontryagin space presented in 22]; an advantage of this definition is that it can be used in the selfadjoint 
case as well. The multiplication theorem for characteristic functions from [22] yields an alternative method to obtain the factorization (2.17) of the resolution matrix $W_{[0, j]}(\lambda)$.

Definition A.1 (22]). Let $T$ and $T^{+}$be closed linear relations in a Pontryagin space $\mathfrak{H}$, both contained in the linear relation $S_{*}=T+T^{+}$. A linear mapping $\mathcal{B}=\left(\mathcal{B}_{-}, \mathcal{B}_{+}\right)$: $S_{*} \mapsto \mathcal{H}=\mathbb{C}^{2}$ is called a boundary mapping for the pair $T, T^{+}$if

(i) $\operatorname{ker} \mathcal{B}_{+}=T^{+}, \operatorname{ker} \mathcal{B}_{-}=T, \operatorname{ran} \mathcal{B}_{ \pm}=\mathbb{C}^{2}$;

(ii) $\left[f^{\prime}, g\right]-\left[f, g^{\prime}\right]=i\left\langle\mathcal{B}_{+} \hat{f}, \mathcal{B}_{+} \hat{g}\right\rangle_{\mathcal{H}}-i\left\langle\mathcal{B}_{-} \hat{f}, \mathcal{B}_{-} \hat{g}\right\rangle_{\mathcal{H}} \quad \forall \hat{f}, \hat{g} \in S_{*}$.

Here $\langle\cdot, \cdot\rangle=(J \cdot, \cdot)$ is the indefinite inner product in $\mathcal{H}$ given by a signature operator $J=J^{*}=J^{-1}$. The characteristic function of the linear relation $T$ corresponding to the boundary mapping $\mathcal{B}$ is (see 22] the operator-valued function $W(\lambda)$ defined by the formula

$$
W(\lambda) \mathcal{B}_{+} \widehat{f}(\lambda)=\mathcal{B}_{-} \widehat{f}(\lambda), \quad \widehat{f}(\lambda) \in \mathfrak{N}_{\lambda}(S), \quad \lambda \in \rho(T) .
$$

Proposition A.2. The characteristic function of a linear relation

$$
T_{[l, j]}=\left\{\widehat{f}=\left\{f, H_{[l, j]} f+\widetilde{b}_{j} f_{j+1} e_{j, 0}\right\}:\left[f, e_{l, 0}\right]=0\right\},
$$

corresponding $($ for $j>l)$ to the boundary mapping $\mathcal{B}=\left(\mathcal{B}_{+}, \mathcal{B}_{-}\right)$,

$$
\begin{gathered}
\mathcal{B}_{-} \widehat{f}=\left(\begin{array}{c}
\varepsilon_{l} f_{l} \\
-b_{l-1} f_{l-1}
\end{array}\right), \quad \mathcal{B}_{+} \widehat{f}=\left(\begin{array}{c}
\varepsilon_{j+1} f_{j+1} \\
-b_{j} f_{j}
\end{array}\right), \\
\widehat{f}=\left\{f, H_{[l, j]} f+b_{l-1} f_{l-1} e_{l, 0}+\widetilde{b}_{j} f_{j+1} e_{j, 0}\right\} \in S_{[l, j] *}=T_{[l, j]}+T_{[l, j]}^{+},
\end{gathered}
$$

and the signature operator

$$
J=\left(\begin{array}{rr}
0 & -i \\
i & 0
\end{array}\right),
$$

coincides with the resolution matrix $W_{[l, j]}(\lambda)$.

Proof. Consider first the case $j>l$. Set $S_{*}=T+T^{+}$. Then the subspace $\mathfrak{N}_{\lambda}\left(S_{*}\right)$ for the linear relation

$$
S_{*}=\left\{\widehat{f}=\left\{f, H_{[l, j]} f+b_{l-1} f_{l-1} e_{l, 0}+\widetilde{b}_{j} f_{j+1} e_{j, 0}\right\}: f_{l-1}, f_{j+1} \in \mathbb{C}\right\}
$$

consists of vectors

$$
\begin{aligned}
\widehat{f}_{\lambda} & =\left\{f_{\lambda}, \lambda f_{\lambda}\right\}=c_{1} \widehat{\pi}_{[l, j]}(\lambda)+c_{2}\left(\widehat{\xi}_{[l, j]}(\lambda)-\left\{0, e_{l, 0}\right\}\right) \\
& =\left\{c_{1} \pi_{[l, j]}(\lambda)+c_{2} \xi_{[l, j]}(\lambda), H_{[l, j]} f_{\lambda}+\varepsilon_{j} b_{j}\left(P_{j+1}^{(l)}(\lambda) c_{1}+Q_{j+1}^{(l)}(\lambda) c_{2}\right) e_{j, 0}-c_{2} e_{l, 0}\right\},
\end{aligned}
$$

where $c_{1}, c_{2} \in \mathbb{C}$. Taking into account (A.3), (3.71), and (3.72) we have

$$
\mathcal{B}_{-} \widehat{f}_{\lambda}=\left(\begin{array}{l}
c_{1} \\
c_{2}
\end{array}\right), \quad \mathcal{B}_{+} \widehat{f}_{\lambda}=\left(\begin{array}{cc}
P_{j+1}^{(l)}(\lambda) & Q_{j+1}^{(l)}(\lambda) \\
-\varepsilon_{j} b_{j} P_{j}^{(l)}(\lambda) & -\varepsilon_{j} b_{j} Q_{j}^{(l)}(\lambda)
\end{array}\right)\left(\begin{array}{l}
c_{1} \\
c_{2}
\end{array}\right) .
$$

Together with (3.54) and (3.60), this formula shows that

$$
W(\lambda)=\left(\begin{array}{cc}
P_{j+1}^{(l)}(\lambda) & Q_{j+1}^{(l)}(\lambda) \\
-\varepsilon_{j} b_{j} P_{j}^{(l)}(\lambda) & -\varepsilon_{j} b_{j} Q_{j}^{(l)}(\lambda)
\end{array}\right)^{-1}=\mathcal{W}_{[l, j]}(\lambda) .
$$


Remark A.3. In the case $j=l$ we set

$$
S_{[l, l] *}=\left\{\widehat{f}=\left\{f, H_{[l, l]} f+\widetilde{b}_{l} f_{l+1} e_{l, 0}\right\}: f_{l+1} \in \mathbb{C}\right\}
$$

and define the multivalued boundary mapping $\mathcal{B}=\left(\mathcal{B}_{-}, \mathcal{B}_{+}\right)$by the formula

$$
\mathcal{B}_{-} \widehat{f}=\left(\begin{array}{c}
\varepsilon_{l} f_{l} \\
\varphi
\end{array}\right), \quad \mathcal{B}_{+} \widehat{f}=\left(\begin{array}{c}
\left(\varphi / \widetilde{b}_{l}+f_{l+1}\right) \varepsilon_{l+1} \\
-b_{l} f_{l}
\end{array}\right), \quad \widehat{f} \in S_{[l, l] *} .
$$

Each vector $\widehat{f}_{\lambda} \in \mathfrak{N}_{\lambda}\left(S_{[l, l] *}\right)$ can be represented in the form

$$
\widehat{f}_{\lambda}=c\left\{\pi_{[l, l]}(\lambda), H_{[l, l]} \pi_{[l, l]}(\lambda)+\varepsilon_{l} b_{l} P_{l+1,0}^{(l)}(\lambda) e_{l, 0}\right\},
$$

where $c \in \mathbb{C}$. Applying $\mathcal{B}_{ \pm}$defined by (A.3) we get

$$
\mathcal{B}_{-} \widehat{f}_{\lambda}=\left(\begin{array}{c}
c \\
\varphi
\end{array}\right), \quad \mathcal{B}_{+} \widehat{f}=\left(\begin{array}{c}
\varepsilon_{l} \varphi / b_{l}+P_{l+1}^{(l)}(\lambda) c \\
-\varepsilon_{l} b_{l} c
\end{array}\right), \quad \widehat{f} \in S_{[l, l] *} .
$$

Therefore,

$$
W(\lambda)=\left(\begin{array}{cc}
p_{l}(\lambda) / b_{l} & \varepsilon_{l} / b_{l} \\
-\varepsilon_{l} b_{l} & 0
\end{array}\right)^{-1}=\left(\begin{array}{cc}
0 & -\varepsilon_{l} / b_{l} \\
\varepsilon_{l} b_{l} & p_{l}(\lambda) / b_{l}
\end{array}\right)=\mathcal{W}_{[l]}(\lambda) .
$$

Now we prove a theorem on the factorization of the resolution matrix using the multiplication theorem for the colligation of two relations [22].

Theorem A.4. For $0 \leq l<j$ the linear relation $T_{[0, j]}$ is the colligation of the linear relations $T_{[0, l]}$ and $T_{[l+1, j]}$ given by

$$
T_{[0, j]}=\left\{\widehat{f}^{(1)} \oplus \widehat{f}^{(2)} \in S_{[0, l] *} \oplus S_{[l+1, j] *}: \mathcal{B}_{+}^{1} \widehat{f}^{(1)}-\mathcal{B}_{-}^{2} \widehat{f}^{(2)}=\mathcal{B}_{-}^{1} \widehat{f}^{(1)}=0\right\} .
$$

Moreover,

$$
\mathcal{W}_{[0, j]}(\lambda)=\mathcal{W}_{[0, l]}(\lambda) \mathcal{W}_{[l+1, j]}(\lambda) .
$$

Proof. By (A.3), equalities $\mathcal{B}_{+}^{1} \widehat{f}^{(1)}=\mathcal{B}_{-}^{2} \widehat{f}_{2}$ and $\mathcal{B}_{-}^{1} \widehat{f}^{(1)}=0$ take the form

$$
f_{l+1}^{(1)}=f_{l+1}^{(2)}, \quad f_{l}^{(1)}=f_{l}^{(2)}, \quad f_{-1}^{(1)}=f_{0}^{(1)}=0,
$$

where

$$
\begin{aligned}
& \widehat{f}^{(1)}=\left\{f^{(1)}, H_{[0, l]} f^{(1)}+f_{-1}^{(1)} e_{0,0}+\widetilde{b}_{l} f_{l+1}^{(1)} e_{l, 0}\right\} \in S_{[0, l] *}, \\
& \widehat{f}^{(2)}=\left\{f^{(2)}, H_{[l+1, j]} f^{(2)}+b_{l} f_{l}^{(2)} e_{l+1,0}+\widetilde{b}_{j} f_{j+1}^{(2)} e_{j, 0}\right\} \in S_{[l+1, j] *} .
\end{aligned}
$$

One can easily see that the vector $\widehat{f}=\widehat{f}^{(1)} \oplus \widehat{f}^{(2)}$ satisfies the relations

$$
\widehat{f}=\left\{f, H_{[0, j]} f+\widetilde{b}_{j} f_{j+1}^{(2)} e_{j, 0}\right\}, \quad\left[f, e_{0,0}\right]=0,
$$

which means that $\widehat{f} \in T_{[0, j]}$.

Now the statement on the factorization of the characteristic function $W_{[0, j]}(\lambda)$ follows from [22, Theorem 4].

In the case of elementary generalized Jacobi matrices the result on colligations is proved similarly using the boundary mapping (A.5). 


\section{REFERENCES}

[1] A. I. Aptekarev, V. A. Kaliaguine, and W. Van Assche, Criterion for the resolvent set of nonsymmetric tridiagonal operators. Proc. Amer. Math. Soc. 123 (1995), 2423-2430. MR1254830 (96c:47041)

[2] N. I. Akhiezer, The classical moment problem and some related questions in analysis. Fizmatgiz, Moscow, 1961; English transl., Hafner Publishing Co., New York, 1965. MR0184042 (32:1518)

[3] D. Alpay, A. Dijksma, and H. Langer, Factorization of J-unitary matrix polynomials on the line and a Schur algorithm for generalized Nevanlinna functions. Linear Algebra Appl. 387 (2004), 313-342. MR2069282 (2005b:47029)

[4] D. Alpay, T. Azizov, A. Dijksma, and H. Langer, The Schur algorithm for generalized Schur functions, III: J-unitary matrix polynomials on the circle. Linear Algebra Appl. 369 (2003), 113-144. MR.1988481 (2005e:47032)

[5] F. V. Atkinson, Discrete and continuous boundary problems. Academic Press, New York-London, 1964. MR0176141 (31:416)

[6] T. Ya. Azizov and I. S. Iokhvidov, Linear operators in spaces with an indefinite metric. "Nauka", Moscow, 1986; English transl., Wiley, Chichester, 1989. MR1033489 (90j:47042)

[7] B. Beckermann, Complex Jacobi matrices. J. Comput. Appl. Math. 127 (2001), 17-65. MR1808568 (2001k:39030)

[8] G. Baker, J. L. Gammel, and J. G. Wills, An investigation of the applicability of the Padé approximant method. J. Math. Anal. Appl. 2 (1961), 405-418. MR0130093 (23:B3125)

[9] G. Baker and P. Graves-Morris, Padé approximants. Parts I, II. Addison-Wesley Publishing, Reading, MA, 1981. MR635619 (83a:41009a)

[10] Yu. M. Berezanskii, Expansions in eigenfunctions of selfadjoint operators. "Naukova Dumka", Kiev, 1965; English transl., Amer. Math. Soc., Providence, RI, 1968. MR0222718 (36:5768)

[11] V. I. Buslaev, The Baker-Gammel-Wills conjecture in the theory of Padé approximants. Mat. Sb. 193 (2002), no. 6, 25-38; English transl., Sb. Math. 193 (2002), no. 5-6, 811-823. MR1957951 (2004c:41025)

[12] F. R. Gantmacher, The theory of matrices. Vols. 1. 2, AMS-Chelsea Publications, Amer. Math. Soc., Providence, RI, 1959. MR107649 (21:6372c)

[13] F. Gesztesy and B. Simon, $m$-functions and inverse spectral analysis for finite and semi-infinite Jacobi matrices. J. Anal. Math. 73 1997, 267-297. MR1616422 (99c:47039)

[14] A. A. Gonchar, On convergence of Padé approximants for some classes of meromorphic functions. Mat. Sb. 97 (1975), 607-629; English transl. in Math. USSR-Sb. 26 (1975). MR0387552 (52:8392)

[15] I. Gohberg, P. Lancaster, and L. Rodman, Matrix polynomials. Academic Press, New York, 1982. MR662418 (84c:15012)

[16] I. C. Gohberg and M. G. Krein, Fundamental aspects of defect numbers, root numbers and indexes of linear operators. Uspehi Mat. Nauk (N.S.) 12 (1957), no. 2, 43-118. (Russian) MR0096978 $(20: 3459)$

[17] V. I. Gorbachuk and M. L. Gorbachuk, Boundary value problems for operator differential equations. "Naukova Dumka", Kiev, 1984; English transl., Kluwer, Dordrecht, 1991. MR1154792 (92m:34133)

[18] A. Dijksma, H. Langer, A. Luger, and Yu. Shondin, A factorization result for generalized Nevanlinna functions of the class $\mathbf{N}_{\kappa}$. Integral Equations Operator Theory 36 (2000), 121-125. MR1736921 (2000i:47027)

[19] M. Derevyagin, On the Schur algorithm for indefinite moment problem. Methods Functional Anal. Topol. 9 (2003), 133-145. MR1999775 (2004j:30075)

[20] M. Derevyagin and V. Derkach, Spectral problems for generalized Jacobi matrices. Linear Algebra Appl. 382 (2004), 1-24. MR2050096 (2005d:47051)

[21] V. Derkach, On generalized resolvents of Hermitian relations in Krein spaces. J. Math. Sci. 97 (1999), no. 5, 4420-4460. MR1728871(2001c:47042)

[22] - On characteristic functions of linear relations and unitary colligations. Dopov. Nats. Akad. Nauk Ukr. Mat. Prirodozn. Tekh. Nauki 2001, no. 11, 28-33. MR1900470 (2003b:47023)

[23] V. A. Derkach, S. Hassi, amd H. S. V. de Snoo, Operator models associated with Kac subclasses of generalized Nevanlinna functions. Methods Functional Anal. Topology, 1999, no. 5. 65-87. MR:1771251 (2001f:47063)

[24] - Generalized Nevanlinna functions with polynomial asymptotic behaviour and regular perturbations. Operator theory in Krein spaces and nonlinear eigenvalue problems, Birkhäuser, Basel, 2001, pp. 169-189. MR1846057(2002j:47042)

[25] V. A. Derkach and M. M. Malamud, The extension theory of Hermitian operators and the moment problem. J. Math. Sci. 73 (1995), no. 2, 141-242. MR1318517(95m:47009) 
[26] W. B. Jones and W. J. Thron, Continued fractions. Analytic theory and applications. AddisonWesley, Reading, MA, 1980. MR595864 (82c:30001)

[27] H. Dym, On Hermitian block Hankel matrices, matrix polynomials, the Hamburger moment problem, interpolation and maximum entropy Integral Equations Operator Theory 12 (1989), 757-811. MR:1018213 (91c:30065)

[28] I. S. Iohvidov, M. G. Krein, and H. Langer, Introduction to the spectral theory in spaces with an indefinite metric. Akademie Verlag,, Berlin, 1982. MR691137 (85g:47050)

[29] M. G. Krein, The fundamental propositions of the theory of representations of Hermitian operators with deficiency index $(m, m)$. Ukrain. Mat. Zh. 1 (1949), no. 2, 3-66. (Russian) MR0048704(14:56d)

[30] M. G. Krein and H. Langer, On defect subspaces and generalized resolvents of a Hermitian operator in the space $\Pi_{\kappa}$. Functional Anal. Appl. 5 (1971), 136-146. MR0282238 (43:7951a) MR0282238 (43:7951b)

[31] Uber die Q-Funktion eines $\pi$-hermiteschen Operators im Raume $\Pi_{\kappa}$. Acta. Sci. Math. (Szeged) 34 (1973), 191-230. MR0318958(47:7504)

[32] _ Über einige Fortsetzungsprobleme, die eng mit der Theorie hermitescher Operatoren im Raume $\Pi_{\kappa}$ zusammenhängen. I. Math. Nachr. 77 (1977), 187-236. MR0461188(57:1173)

[33] _ _ Über einige Fortsetzungsprobleme, die eng mit der Theorie hermitescher Operatoren im Raume $\Pi_{\kappa}$ zusammenhängen. II. J. Funct. Anal. 30 (1978), no. 3, 390-447. MR0518342(80h:47045)

[34] _ On some extension problems which are closely connected with the theory of Hermitian operators in a space $\Pi_{\kappa}$. III. Indefinite analogues of the Hamburger and Stieltjes moment problems, Part I, Beiträge zur Anal. Vol. 14, 1979, pp. 25-40. MR0563344 (83b:47047a)

[35] _ Some propositions of analytic matrix functions related to the theory of operators in the space $\Pi_{\kappa}$. Acta Sci. Math. (Szeged) 43 (1981), 181-205. MR0621369(82i:47053)

[36] M. G. Krein and A. A. Nudelman. The Markov moment problem and extremal problems. "Nauka", Moscow, 1973; English transl., Amer. Math. Soc., Providence, RI, 1977. MR0458081 (56:16284)

[37] M. G. Krein amd Sh. N. Saakjan, The resolvent matrix of a Hermitian operator and the characteristic functions connected with it. Funkcional. Anal. i Prilozhen. 4 (1970) no. 3, 103-104; English transl. in Functional Anal. Appl. 4 (1970). MR0275205 (43:962)

[38] H. Langer, A characterization of generalized zeros of negative type of functions of the class $\mathbf{N}_{\kappa}$. Oper. Theory Adv. Appl., vol. 17, 1986, pp. 201-212. MR901070 (88j:47051)

[39] H. Langer and B. Textorius, On generalized resolvents and Q-functions of symmetric linear relations (subspaces) in Hilbert space. Pacific J. Math. 72 (1977), 135-165. MR0463964 (57:3902)

[40] D. S. Lubinsky, Rogers-Ramanujan and the Baker-Gammel-Wills (Padé) conjecture. Ann. of Math. (2) 157 (2003), 847-889. MR1983783 (2004b:30071)

[41] A. Magnus, Certain continued fractions associated with the Padé table. Math. Z. 78 (1962), 361-374. MR0150271(27:272)

[42] _ Expansion of power series into P-fractions. Math. Z. 80 (1962), 209-216. MR0150272 $(27: 273)$

[43] E. M. Nikishin and V. N. Sorokin, Rational approximations and orthogonality. "Nauka", Moscow, 1988; English transl., Amer. Math. Soc., Providence, RI, 1991. MR.1130396 (92i:30037)

[44] E. A. Rahmanov, The convergence of diagonal Padé approximants. Mat. Sb. 104 (1977), no. 2, 271-291; English transl., Math. USSR-Sb. 33 (1977), no. 2, 243-260. MR0492292 (58:11430)

[45] B. Simon, The classical moment problem as a self-adjoint finite difference operator. Adv. Math. 137 (1998), 82-203. MR1627806 (2001e:47020)

[46] H. Stahl, On the divergence of certain Padé approximants and the behaviour of the associated orthogonal polynomials. Polynômes Orthogonaux et Applications, Lect. Notes Math. vol. 1171, Springer-Verlag. Heidelberg, 1985. pp. 321-330. MR.839001 (87g:41037)

[47] H. van Rossum, Padé approximants and indefinite inner product spaces. Padé and rational approximation. Theory and applications, E. B. Saff and R. S. Varga (eds.), Tampa, 1976, pp. 111-119. $\operatorname{MR} 0617938(58: 29725)$

Donetsk National University, Universitetskaya 24, 83055 Donetsk, Ukraine

E-mail address: derkach.v@gmail.com

Donetsk National University, Universitetskaya 24, 83055 Donetsk, Ukraine

E-mail address: derevyagin.m@gmail.com

Translated by O. A. KHLEBORODOVA 